DOI 10.4171/JEMS/789

Diego Armentano · Carlos Beltrán · Peter Bürgisser

Felipe Cucker · Michael Shub

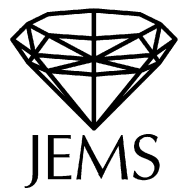

\title{
A stable, polynomial-time algorithm for the eigenpair problem
}

Received May 8, 2015

\begin{abstract}
We describe algorithms for computing eigenpairs (eigenvalue-eigenvector pairs) of a complex $n \times n$ matrix $A$. These algorithms are numerically stable, strongly accurate, and theoretically efficient (i.e., polynomial-time). We do not believe they outperform in practice the algorithms currently used for this computational problem. The merit of our paper is to give a positive answer to a long-standing open problem in numerical linear algebra.
\end{abstract}

Keywords. Eigenvalue computations, homotopy methods

So the problem of devising an algorithm [for the eigenvalue problem] that is numerically stable and globally (and quickly!) convergent remains open.

J. Demmel [25, p. 139]

\section{Introduction}

\subsection{The problem}

The quotation from Demmel opening this article, though possibly puzzling for those who day-to-day satisfactorily solve eigenvalue problems, summarizes a long-standing open problem in numerical linear algebra. The first algorithm that comes to mind for computing eigenvalues - to compute the characteristic polynomial $\chi_{A}$ of $A$ and then compute (i.e., approximate) its zeros_ - has proved to be numerically unstable. The so called Wilkinson's polynomial,

$$
w(x):=\prod_{i=1}^{20}(x-i)=x^{20}+w_{19} x^{19}+\cdots+w_{1} x+w_{0},
$$

D. Armentano: Universidad de la República, Montevideo, Uruguay; e-mail: diego@cmat.edu.uy

C. Beltrán: Universidad de Cantabria, Santander, Spain; e-mail: beltranc@ unican.es

P. Bürgisser: Technische Universität Berlin, Berlin, Germany; e-mail: pbuerg@ math.tu-berlin.de

F. Cucker: City University of Hong Kong, Hong Kong; e-mail: macucker@ cityu.edu.hk

M. Shub: City University of New York, New York, U.S.A.; e-mail: shub.michael@gmail.com

Mathematics Subject Classification (2010): 65F15, 65Y20, 65H17 
is often used to illustrate this fact. For a diagonal matrix $D$ with diagonal entries $1,2, \ldots, 20$ (and therefore with $\chi_{D}(x)=w(x)$ ) an error of $2^{-23}$ in the computation of $w_{19}=-210$ produces, even if the rest of the computation is done error-free, catastrophic variations in the zeros of $\chi_{D}$. For instance, the zeros at 18 and 19 collide into a double zero close to 18.62 , which will unfold into two complex conjugate zeros if the error in $w_{19}$ is further increased. And yet, there is nothing wrong in the nature of $D$ (in numerical analysis terms, as we will see below, $D$ is a well-conditioned matrix for the eigenvalue problem). The trouble appears to lie in the method.

Barred from using this immediate algorithm due to its numerical instability, researchers devoted efforts to come up with alternative methods which would appear to be stable. Among those proposed, the one that is today's algorithm of choice is the iterated QR with shifts. This procedure behaves quite efficiently in general and yet, as Demmel pointed out in 1997 [25, p. 139],

after more than 30 years of dependable service, convergence failures of this algorithm have quite recently been observed, analyzed, and patched [...]. But there is still no global convergence proof, even though the current algorithm is considered quite reliable.

Our initial quotation followed these words in Demmel's text. It asked for an algorithm which will be numerically stable and for which convergence, and if possible small complexity bounds, can be established. Today, more than 20 years after Demmel's text, this demand retains all of its urgency: it is not known if any of the standard numerical linear algebra algorithms satisfies the properties above. For example:

- The unshifted QR algorithm terminates with probability 1 but is probably infinite average cost if approximations to the eigenvectors are to be output (see [32]).

- The QR algorithm with Rayleigh Quotient shift fails for open sets of real input matrices (see $[8,9])$.

- We do not know whether the Francis (double) shift algorithm converges generally on real or complex matrices, nor an estimate of its average cost.

- Other algorithms in modern texts are analyzed but do not estimate the (necessarily infinite in the worst case) number of iterations, which usually relies on experimental results; see for example [40] which uses a divide and conquer algorithm or [26] which is in turn based on the algorithm in [6].

Algorithms which output approximate eigenvalues without accompanying approximate eigenvectors might be easier to analyze. The experimental evidence of [41] for symmetric matrices suggests that many of the algorithms in use are of average finite cost and even that there is some universality. An informal explanation of this fact is that the eigenvalues of symmetric matrices are very well conditioned: see for example [56, eq. (1.5)]. But eigenvectors are another matter. When the matrices are close to having multiple eigenvalues, the condition of the eigenvector tends to infinity. For example, even for $2 \times 2$ symmetric matrices, any pair of orthogonal vectors $(a, b)$ and $(-b, a)$ are the eigenvectors of a matrix

$$
\left(\begin{array}{cc}
1+\varepsilon_{1} & \varepsilon_{3} \\
\varepsilon_{3} & 1+\varepsilon_{2}
\end{array}\right)
$$

for $\left|\varepsilon_{i}\right|, i=1,2,3$, arbitrarily small. 
The only goal of this paper is to give a positive answer to Demmel's question. The set of our main results can be informally stated as follows.

Main results. We exhibit algorithms which on input a complex matrix A with complex Gaussian entries generate (with probability 1) an "excellent" approximation to one of (or all) the (eigenvalue, eigenvector) pairs of A. Some of these algorithms are deterministic while some are randomized. Their running time (expected running time for the randomized case) is polynomial in $n$ on average (with respect to A) as well as under a standard smoothed analysis.

More precisely, the average complexity bounds we prove, for $n \times n$ matrices, are $\mathcal{O}\left(n^{7}\right)$ for the computation of a single eigenpair with either a deterministic or a randomized algorithm, and $\mathcal{O}\left(n^{9}\right)$ - for the computation of all eigenpairs with a deterministic algorithm. Note that these are just upper bounds: the practical performance of the algorithms might be better (see $\$ 2.10$ ). The precise statements of the main results are in Theorems $2.25,2.28$, and 2.35 .

\subsection{A few words on approximations}

It must be said upfront that we do not think the algorithm we propose will outperform, in general, iterated QR with shifts. It nonetheless possesses some worthy features which we want to describe in this introduction. The key one, already mentioned, is that both convergence and complexity bounds can be established for it. It is also numerically stable. But in addition, it is strongly accurate.

A starting point to understand the meaning of this last claim is the observation that there are two different obstructions to the exact computation of an eigenpair. Firstly, the use of finite precision, and the ensuing errors accumulating during the computational process. The expression numerically stable is usually vested on algorithms for which this accumulated error on the computed quantities is not much larger than that resulting from an error-free computation on an input datum which has been read (and approximated) with machine precision. Secondly, the nonlinear character of the equations defining eigenvalues and eigenvectors in terms of the given matrix. For $n \geq 5$, we learned from Abel and Galois that we cannot write down these eigenvalues in terms of the matrix entries, not even using radicals, and the same remains true for eigenvectors. Hence, we can only compute approximations of them and this is so even assuming infinite precision in the computation.

The expression strongly accurate refers to the quality of these approximations. It is common to find in the literature (at least) three notions of approximation which we next briefly describe. To simplify, we illustrate with the computation of a value $\zeta \in \mathbb{C}$ from a datum $A \in \mathbb{C}^{N}$ (and the reader may well suppose that this computation is done with infinite precision). We let $\widetilde{\zeta}$ be the quantity actually computed and we consider the following three requirements on it:

Backward approximation. The element $\tilde{\zeta}$ is the solution of a datum $\widetilde{A}$ close to $A$. Given $\varepsilon>0$, we say that $\tilde{\zeta}$ is an $\varepsilon$-backward approximation when $\|A-\widetilde{A}\| \leq \varepsilon$ (resp. $\|A-\widetilde{A}\| \leq \varepsilon\|A\|$ if we are interested in relative errors). 
Forward approximation. The quantity $\tilde{\zeta}$ is close to $\zeta$. Given $\varepsilon>0$, we say that $\tilde{\zeta}$ is an $\varepsilon$-forward approximation when $|\zeta-\widetilde{\zeta}| \leq \varepsilon(\operatorname{resp} .|\zeta-\widetilde{\zeta}| \leq \varepsilon|\zeta|)$.

Approximation à la Smale. An appropriate version of Newton's iteration, starting at $\widetilde{\zeta}$, converges immediately, quadratically fast, to $\zeta$, either in absolute or relative error.

These requirements are often, but not always, increasingly demanding. For instance, if $\zeta$ is an $\varepsilon$-backward approximation then the forward error $|\zeta-\widetilde{\zeta}|$ is bounded, roughly, by $\varepsilon \operatorname{cond}(A)$. Here cond $(A)$ is the condition number of $A$, a quantity usually greater than 1 . So, in general, $\varepsilon$-backward approximations are not $\varepsilon$-forward approximations, and if $A$ is poorly conditioned, $\widetilde{\zeta}$ may be a poor forward approximation of $\zeta$ (we note, however, that backward approximations do not necessarily exist, and condition numbers smaller than 1 do occur). We also observe that if $\widetilde{\zeta}$ is an approximation à la Smale we can obtain an $\varepsilon$-forward approximation from $\widetilde{\zeta}$ by performing $\mathcal{O}(\log |\log \varepsilon|)$ Newton's steps (see for example Theorem 1.1 below). To obtain an approximation à la Smale from an $\varepsilon$-forward approximation we need, in contrast, that $\varepsilon$ will be of the order of the inverse of the condition of the zero (see Theorem 2.12 below).

When we say that our algorithm is strongly accurate, we refer to the fact that the returned eigenpairs are approximations à la Smale of true eigenpairs.

In our case, we can not only efficiently compute $\varepsilon$-forward approximations as above but also with respect to relative error. These are pairs $(\zeta, w)$ satisfying

$$
d_{\mathbb{P}}(w, v) \leq \varepsilon \quad \text { and } \quad|\zeta-\lambda| \leq \varepsilon|\lambda|
$$

for some true eigenpair $(\lambda, v)$ of $A$. Here $d_{\mathbb{P}}(w, v)$ denotes the angle between $w$ and $v$ (note that here the scaling of eigenvectors renders the relativization of the error moot).

Theorem 1.1. We exhibit an algorithm that, given a matrix $A \in \mathbb{C}^{n \times n}$, an approximate eigenpair $(\zeta, w)$ returned by any of the algorithms in the main results, and an accuracy $\varepsilon \in(0,1 / 2)$, produces an $\varepsilon$-forward approximation (in relative error) of the approximated true eigenpair $(\lambda, v)$. The algorithm terminates provided $\lambda \neq 0$. Its average cost over Gaussian matrices $A$ (independently of the chosen approximate eigenpair) is $\mathcal{O}\left(n^{3} \log _{2} \log _{2}(n / \varepsilon)\right)$.

Combining our main results with Theorem 1.1 we can thus compute $\varepsilon$-forward approximations (in relative error) of all eigenpairs of random Gaussian matrices with average running time $\mathcal{O}\left(n^{9}+n^{3} \log _{2} \log _{2}(n / \varepsilon)\right)$. See $\S 11$ for a proof of Theorem 1.1.

\subsection{A few words on complexity}

The cost, understood as the number of arithmetic operations performed, of computing an approximation of an eigenpair for a matrix $A \in \mathbb{C}^{n \times n}$, depends on the matrix $A$ itself. Actually, and this is a common feature in numerical analysis, it depends on the condition number cond $(A)$ of the matrix $A$. But this condition number is not known a priori. It was therefore advocated by Smale [51] to eliminate this dependency in complexity bounds by endowing data space with a probability distribution and estimating expected costs. This idea has its roots in early work of Goldstine and von Neumann [57]. 
In our case, data space is $\mathbb{C}^{n \times n}$, and a common probability measure to endow it with is the standard Gaussian. Expectations of cost with respect to this measure yield expressions in $n$ usually referred to as average cost. A number of considerations, including the suspicion that the use of the standard Gaussian could result in complexity bounds which are too optimistic compared with "real life", prompted Spielman and Teng to introduce a different form of probabilistic analysis, called smoothed analysis. In this, one replaces the average analysis' goal of showing that

for a random $A$ it is unlikely that the cost for $A$ will be large

by

for all $\hat{A}$, it is unlikely that a slight random perturbation $A=\hat{A}+\Delta A$ will require

a large cost.

The expectations obtained for a smoothed analysis will now be functions of both the dimension $n$ and some measure of dispersion for the random perturbations (e.g., a variance).

Smoothed analysis was first used for the simplex method of linear programming [54]. Some survey expositions of its rationale are in $[53,55,17]$. One may argue that it has been well accepted by the scientific community from the fact that Spielman and Teng were awarded the Gödel 2008 and Fulkerson 2009 prizes for it (the former by the theoretical computer science community and the latter by the optimization community). Also, in 2010, Spielman was awarded the Nevanlinna prize, and smoothed analysis appears in the laudatio of his work.

In this paper we will exhibit bounds for the cost of our algorithm both for average and smoothed analyses.

\subsection{A few words on numerical stability}

The algorithm we deal with in this paper belongs to the class of homotopy continuation methods. Experience has shown that algorithms in this class are very stable and stability analyses have been done for some of them, e.g. [16, 11, 23]. Because of this, we will assume infinite precision all along this paper and steer clear of any form of stability analysis. We nonetheless observe that such an analysis can be easily carried out following the steps in the papers mentioned above.

\subsection{Previous and related work}

Homotopy continuation methods go back, at least, to the work of Lahaye [33]. A detailed survey of their use in solving polynomial equations is in [35]. More explicit focus on eigenvalue computations is considered in [21, 36, 37, 38] but we do not know of any serious attempt to implement them.

In the early 1990s Shub and Smale set up a program to understand the cost of solving square systems of complex polynomial equations using homotopy methods. In a collection of articles [45, 46, 47, 48, 49], known as the Bézout series, they put in place many of the notions and techniques that occur in this article. The Bézout series did not, however, 
conclusively settle the understanding of the cost above, and in 1998 Smale proposed it as the 17 th in his list of problems for the mathematicians of the 21 st century [52]. A probabilistic solution to this problem was found in $[12,13]$, then a deterministic quasipolynomial solution was described in [18]. Finally, a deterministic polynomial solution was recently found by Lairez [34] and is now considered fully answered by the community.

The results in these papers cannot be directly used for the eigenpair problem since instances of the latter are ill-posed as polynomial systems. But the intervening ideas can be reshaped to attempt a tailor-made analysis for the eigenpair problem. A major step in this direction was done by Armentano in his $\mathrm{PhD}$ thesis (see [3] and its precedent [24]), where the condition number $\mu$ for the eigenpair problem was exhaustively studied. A further step was taken in [5] where $\mu$ was used to analyze a randomized algorithm for the Hermitian eigenpair problem.

Our paper follows this stream of research.

Since the appearance of our work, Paul Breiding [15] has successfully generalized the ideas of our paper to the computation of eigenpairs of homogeneous polynomial systems.

\subsection{Structure of the exposition}

The remainder of this paper is divided into two parts. In the first one, $\$ 2$ below, we introduce all the technical preliminaries, we describe with details the algorithms, and we state our main results (Theorems 2.25, 2.28, and 2.35). The condition number $\mu$, Newton's method, the notion of approximate eigenpair, and Gaussian distributions are among these technical preliminaries. The second part, which occupies us in the subsequent sections, is devoted to proofs. Some short proofs are included in $\$ 2$ as well.

\section{Preliminaries, basic ideas, and main result}

\subsection{Spaces and metric structures}

Let $\mathbb{C}^{n \times n}$ be the set of $n \times n$ complex matrices. We endow this complex linear space with the Frobenius Hermitian product $\langle,\rangle_{F}$ and the associated Frobenius norm $\|\cdot\|_{F}$ given by

$$
\langle A, B\rangle_{F}:=\operatorname{trace}\left(B^{*} A\right)=\sum_{i, j=1}^{n} a_{i j} \overline{b_{i j}}, \quad\|A\|_{F}=\langle A, A\rangle_{F}^{1 / 2},
$$

where $A=\left(a_{i j}\right)$ and $B=\left(b_{i j}\right)$. The unit sphere will be denoted by $\mathbb{S}\left(\mathbb{C}^{n \times n}\right)$ or simply by $\mathbb{S}$. We endow the product vector space $\mathbb{C}^{n \times n} \times \mathbb{C}$ with the canonical Hermitian inner product structure and its associated norm structure and (Euclidean) distance.

The space $\mathbb{C}^{n}$ is equipped with the canonical Hermitian inner product $\langle$,$\rangle . We denote$ by $\mathbb{P}\left(\mathbb{C}^{n}\right)$ the associated projective space. This is a smooth manifold which carries a natural Riemannian metric, namely, the real part of the Fubini-Study metric on $\mathbb{P}\left(\mathbb{C}^{n}\right)$. The Fubini-Study metric is the Hermitian structure on $\mathbb{P}\left(\mathbb{C}^{n}\right)$ given in the following way: for $x \in \mathbb{C}^{n}$,

$$
\left\langle w, w^{\prime}\right\rangle_{x}:=\frac{\left\langle w, w^{\prime}\right\rangle}{\|x\|^{2}}
$$


for all $w, w^{\prime}$ in the Hermitian complement $x^{\perp}=\left\{v \in \mathbb{C}^{n} \mid\langle x, v\rangle=0\right\}$ of $x$ in $\mathbb{C}^{n}$. We denote by $d_{\mathbb{P}}$ the associated Riemannian distance. An explicit formula for that distance (see for example [14, p. 226]) is

$$
d_{\mathbb{P}}(v, w)=\arccos \frac{|\langle v, w\rangle|}{\|v\| \cdot\|w\|} .
$$

Note that this formula makes sense for $v, w \in \mathbb{C}^{n}$ (as the distance between the respective projective classes).

The space $\mathbb{C}^{n \times n} \times \mathbb{C} \times \mathbb{P}\left(\mathbb{C}^{n}\right)$ is endowed with the Riemannian product structure. The resulting distance equals

$$
\operatorname{dist}\left((A, \lambda, v),\left(A^{\prime}, \lambda^{\prime}, v^{\prime}\right)\right)^{2}:=\left\|A-A^{\prime}\right\|_{F}^{2}+\left|\lambda-\lambda^{\prime}\right|^{2}+d_{\mathbb{P}}\left(v, v^{\prime}\right)^{2} .
$$

We will only use this distance on $\mathbb{S} \times \mathbb{C} \times \mathbb{P}\left(\mathbb{C}^{n}\right)$. Note that for $A, A^{\prime} \in \mathbb{S}$, the distance $\operatorname{dist}\left((A, \lambda, v),\left(A^{\prime}, \lambda^{\prime}, v^{\prime}\right)\right)$ is smaller than or equal to the natural geodesic (product) distance in $\mathbb{S} \times \mathbb{C} \times \mathbb{P}\left(\mathbb{C}^{n}\right)$. For any nonzero matrix $A \in \mathbb{C}^{n \times n}$ (not necessarily of unit norm) we will write

$$
\operatorname{dist}_{A}\left((\lambda, v),\left(\lambda^{\prime}, v^{\prime}\right)\right)^{2}:=\frac{\left|\lambda-\lambda^{\prime}\right|^{2}}{\|A\|_{F}^{2}}+d_{\mathbb{P}}\left(v, v^{\prime}\right)^{2} .
$$

Note that for any nonzero $A \in \mathbb{C}^{n \times n}$, $\operatorname{dist}_{A}$ is a distance function in $\mathbb{C} \times \mathbb{P}\left(\mathbb{C}^{n}\right)$, and if $A \in \mathbb{S}$, then $\operatorname{dist}_{A}\left((\lambda, v),\left(\lambda^{\prime}, v^{\prime}\right)\right)=\operatorname{dist}\left((A, \lambda, v),\left(A, \lambda^{\prime}, v^{\prime}\right)\right)$.

\subsection{The varieties $\mathcal{V}, \mathcal{W}, \Sigma^{\prime}$ and $\Sigma$}

We define the solution variety for the eigenpair problem as

$$
\mathcal{V}=\mathcal{V}_{n}:=\left\{(A, \lambda, v) \in \mathbb{C}^{n \times n} \times \mathbb{C} \times \mathbb{P}\left(\mathbb{C}^{n}\right):(A-\lambda \mathrm{Id}) v=0\right\} .
$$

Proposition 2.1 ([3, Proposition 2.2]). The solution variety $\mathcal{V}$ is a smooth submanifold of $\mathbb{C}^{n \times n} \times \mathbb{C} \times \mathbb{P}\left(\mathbb{C}^{n}\right)$, of the same dimension as $\mathbb{C}^{n \times n}$.

The set $\mathcal{V}$ inherits the Riemannian structure of the ambient space. Associated to it there are natural projections:

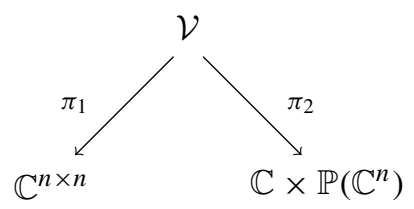

Because of Proposition 2.1, the derivative $D \pi_{1}$ at $(A, \lambda, v)$ is a linear operator between spaces of equal dimension. The subvariety $\mathcal{W}$ of well-posed triples is the subset of triples $(A, \lambda, v) \in \mathcal{V}$ for which $D \pi_{1}(A, \lambda, v)$ is an isomorphism. In particular, if $(A, \lambda, v) \in \mathcal{W}$, the projection $\pi_{1}$ has a branch of its inverse (locally defined) taking $A \in \mathbb{C}^{n \times n}$ to $(A, \lambda, v) \in \mathcal{V}$. 
Let $P_{v^{\perp}}: \mathbb{C}^{n} \rightarrow v^{\perp}$ be the orthogonal projection. Given $(A, \lambda, v) \in \mathbb{C}^{n \times n} \times \mathbb{C} \times$ $\mathbb{P}\left(\mathbb{C}^{n}\right)$, we let $A_{\lambda, v}: v^{\perp} \rightarrow v^{\perp}$ be the linear operator given by

$$
A_{\lambda, v}:=\left.P_{v^{\perp}} \circ(A-\lambda \mathrm{ld})\right|_{v^{\perp}} .
$$

If we choose a representative such that $\|v\|=1$ and we assume that $A_{\lambda, v}$ is invertible, then we have

$$
\begin{aligned}
& i_{\mathbb{C}^{n}} A_{\lambda, v} P_{v^{\perp}}=\left(\mathrm{Id}-v v^{*}\right)(A-\lambda \mathrm{Id})\left(\mathrm{Id}-v v^{*}\right), \\
& i_{\mathbb{C}^{n}} A_{\lambda, v}^{-1} P_{v^{\perp}}=\left(\left(\mathrm{Id}-v v^{*}\right)(A-\lambda \mathrm{Id})\left(\mathrm{Id}-v v^{*}\right)\right)^{\dagger},
\end{aligned}
$$

where $i_{\mathbb{C}^{n}}: v^{\perp} \rightarrow \mathbb{C}^{n}$ is the inclusion and ${ }^{\dagger}$ denotes Moore-Penrose pseudoinverse.

The set of well-posed triples is exactly

$$
\mathcal{W}:=\left\{(A, \lambda, v) \in \mathcal{V}: A_{\lambda, v} \text { is invertible }\right\}
$$

(see [3, Lemma 2.7]). We define $\Sigma^{\prime}:=\mathcal{V} \backslash \mathcal{W}$ to be the variety of ill-posed triples, and $\Sigma=\pi_{1}\left(\Sigma^{\prime}\right) \subset \mathbb{C}^{n \times n}$ the discriminant variety, i.e., the subset of ill-posed inputs.

Remark 2.2. From (7) it is clear that the subset $\Sigma^{\prime}$ is the set of triples $(A, \lambda, v) \in \mathcal{V}$ such that $\lambda$ is an eigenvalue of $A$ of algebraic multiplicity at least 2 . Hence $\Sigma$ is the set of matrices $A \in \mathbb{C}^{n \times n}$ with multiple eigenvalues, and for $A \in \mathbb{C}^{n \times n} \backslash \Sigma$, the eigenvalues of $A$ are pairwise different and $\pi_{1}^{-1}(A)$ is the set of triples $\left(A, \lambda_{1}, v_{1}\right), \ldots,\left(A, \lambda_{n}, v_{n}\right)$, where $\left(\lambda_{i}, v_{i}\right), i=1, \ldots, n$, are the eigenpairs of $A$.

Proposition 2.3 ([19, Proposition 20.18]). The discriminant variety $\Sigma \subset \mathbb{C}^{n \times n}$ is a complex algebraic hypersurface. Consequently, $\operatorname{dim}_{\mathbb{R}} \Sigma=2 n^{2}-2$ for all $n \geq 2$.

\subsection{Unitary invariance}

Let $\mathcal{U}_{n}$ be the group of $n \times n$ unitary matrices. The group $\mathcal{U}_{n}$ naturally acts on $\mathbb{P}\left(\mathbb{C}^{n}\right)$ by $U \cdot w:=U w$. In addition, $\mathcal{U}_{n}$ acts on $\mathbb{C}^{n \times n}$ by conjugation (i.e., $U \cdot A:=U A U^{-1}$ ), and on $\mathbb{C}^{n \times n} \times \mathbb{C}$ by $U \cdot(A, \lambda):=\left(U A U^{-1}, \lambda\right)$. These actions define an action on the product space $\mathbb{C}^{n \times n} \times \mathbb{C} \times \mathbb{P}\left(\mathbb{C}^{n}\right)$, namely,

$$
U \cdot(A, \lambda, v):=\left(U A U^{-1}, \lambda, U v\right) .
$$

Remark 2.4. The varieties $\mathcal{V}, \mathcal{W}, \Sigma^{\prime}$, and $\Sigma$ are invariant under the action of $\mathcal{U}_{n}$ (see [3] for details).

\subsection{Condition of a triple}

In a nutshell, condition numbers measure the worst possible output error resulting from a small perturbation on the input data. More formally, a condition number is the operator norm of the derivative of a solution map such as the branches of $\pi_{1}^{-1}$ mentioned in $\$ 2.1$ above (see $[19, \S 14.1 .2]$ for a general exposition). 
In the case of the eigenpair problem, one can define two condition numbers for the eigenvalue and the eigenvector, respectively, and formulas for both of them have been known at least since [56]. Armentano has shown that one can merge the two in a single one (see $\$ 3$ in [3] for details). Deviating slightly from [3], we define the condition number of $(A, \lambda, v) \in \mathbb{C}^{n \times n} \times \mathbb{C} \times \mathbb{C}^{n}$ as

$$
\mu(A, \lambda, v):=\|A\|_{F}\left\|A_{\lambda, v}^{-1}\right\|
$$

(or $\infty$ if $A_{\lambda, v}$ is not invertible) where $\|\cdot\|$ is the operator norm. This coincides with $\mu_{v}(A, \lambda, v)$ in [3]. Note that from (6), if $(A, \lambda, v)$ is such that $\mu(A, \lambda, v)<\infty$ and $\|v\|=1$, then

$$
\mu(A, \lambda, v)=\|A\|_{F}\left\|\left(\left(\mathrm{Id}-v v^{*}\right)(A-\lambda \mathrm{Id})\left(\mathrm{Id}-v v^{*}\right)\right)^{\dagger}\right\| .
$$

Remark 2.5. The condition number $\mu$ is invariant under the action of the unitary group $\mathcal{U}_{n}$, i.e., $\mu\left(U A U^{-1}, \lambda, U v\right)=\mu(A, \lambda, v)$ for all $U \in \mathcal{U}_{n}$, and scale invariant on the first two components, i.e., $\mu(s A, s \lambda, v)=\mu(A, \lambda, v)$ for all $s \in \mathbb{C} \backslash\{0\}$.

Lemma 2.6 ([3, Lemma 3.8]). For $(A, \lambda, v) \in \mathcal{V}$ we have $\mu(A, \lambda, v) \geq 1 / \sqrt{2}$.

The essence of condition numbers is that they measure how much outputs may vary when inputs are slightly perturbed. The following result, which we will prove in $\S 3$, quantifies this property for $\mu$.

Proposition 2.7. Let $\Gamma:[0,1] \rightarrow \mathcal{V}, \Gamma(t)=\left(A_{t}, \lambda_{t}, v_{t}\right)$, be a smooth curve such that $A_{t}$ lies in the unit sphere of $\mathbb{C}^{n \times n}$ for all $t$. Write $\mu_{t}:=\mu(\Gamma(t))$. Then, for all $t \in[0,1]$,

$$
\left|\dot{\lambda}_{t}\right| \leq \sqrt{1+\mu_{t}^{2}}\left\|\dot{A}_{t}\right\|, \quad\left\|\dot{v}_{t}\right\| \leq \mu_{t}\left\|\dot{A}_{t}\right\|
$$

In particular,

$$
\|\dot{\Gamma}(t)\| \leq \sqrt{6} \mu_{t}\left\|\dot{A_{t}}\right\|
$$

Condition numbers are generally associated to input data. In the case of a problem with many possible solutions (of which returning an eigenpair of a given matrix is a clear case) one can derive the condition of a data from a notion of condition for each of these solutions. A discussion of this issue is given in $[19, \S 6.8]$. For the purposes of this paper, we will be interested in two such derivations: the maximum condition number of $A$,

$$
\mu_{\max }(A):=\max _{1 \leq j \leq n} \mu\left(A, \lambda_{j}, v_{j}\right)
$$

and the mean square condition number of $A$,

$$
\mu_{\mathrm{av}}(A):=\left(\frac{1}{n} \sum_{j=1}^{n} \mu\left(A, \lambda_{j}, v_{j}\right)^{2}\right)^{1 / 2}=\left(\frac{1}{n} \sum_{j=1}^{n}\|A\|_{F}^{2}\left\|A_{\lambda_{j}, v_{j}}^{-1}\right\|^{2}\right)^{1 / 2} .
$$

Condition numbers themselves vary in a controlled manner. The following Lipschitz property and its corollary make this statement precise. 
Theorem 2.8. Let $A, A^{\prime} \in \mathbb{S}$, let $v, v^{\prime} \in \mathbb{C}^{n}$ be nonzero, and let $\lambda, \lambda^{\prime} \in \mathbb{C}$. Suppose that

$$
\mu(A, \lambda, v) \operatorname{dist}\left((A, \lambda, v),\left(A^{\prime}, \lambda^{\prime}, v^{\prime}\right)\right) \leq \frac{\varepsilon}{4 \sqrt{3}} \quad \text { for some } \varepsilon \in(0,1) .
$$

Then

$$
\frac{1}{1+\varepsilon} \mu(A, \lambda, v) \leq \mu\left(A^{\prime}, \lambda^{\prime}, v^{\prime}\right) \leq \frac{1}{1-\varepsilon} \mu(A, \lambda, v) .
$$

Corollary 2.9. Let $A \in \mathbb{S}, A \notin \Sigma$, and let $A^{\prime} \in \mathbb{S}$ be such that

$$
\left\|A-A^{\prime}\right\|_{F} \leq \frac{\varepsilon}{50 \mu_{\max }(A)^{2}} \quad \text { for some } \varepsilon \in(0,1 / 2) .
$$

Then $A^{\prime} \notin \Sigma$ and $(1+\varepsilon)^{-1} \mu_{\max }(A) \leq \mu_{\max }\left(A^{\prime}\right) \leq(1-\varepsilon)^{-1} \mu_{\max }(A)$.

We give the proofs of Theorem 2.8 and Corollary 2.9 in $\S 4$ below.

We close this paragraph observing that restricted to the class of normal matrices, the condition number $\mu$ admits the following elegant characterization.

Lemma 2.10 ([3, Lemma 3.12]). Let $A \in \mathbb{C}^{n \times n} \backslash \Sigma$ be normal, and let $\left(\lambda_{1}, v_{1}\right), \ldots$, $\left(\lambda_{n}, v_{n}\right)$ be its eigenpairs. Then

$$
\mu\left(A, \lambda_{1}, v_{1}\right)=\frac{\|A\|_{F}}{\min _{2 \leq j \leq n}\left|\lambda_{j}-\lambda_{1}\right|} .
$$

\subsection{Newton's method and approximate eigenpairs}

For a nonzero matrix $A \in \mathbb{C}^{n \times n}$, we define the Newton map associated to $A$,

$$
N_{A}: \mathbb{C} \times\left(\mathbb{C}^{n} \backslash\{0\}\right) \rightarrow \mathbb{C} \times\left(\mathbb{C}^{n} \backslash\{0\}\right),
$$

by

$$
N_{A}\left(\begin{array}{l}
\lambda \\
v
\end{array}\right)=\left(\begin{array}{l}
\lambda \\
v
\end{array}\right)-\left(\begin{array}{l}
\dot{\lambda} \\
\dot{v}
\end{array}\right), \quad \text { where }\left(\begin{array}{l}
\dot{\lambda} \\
\dot{v}
\end{array}\right)=\left(\left.D F_{A}(\lambda, v)\right|_{\mathbb{C} \times v^{\perp}}\right)^{-1} F_{A}\left(\begin{array}{l}
\lambda \\
v
\end{array}\right)
$$

and $F_{A}(\lambda, v)=(A-\lambda \mathrm{Id}) v$ is the evaluation map. This is a rational map (it is only defined on an open subset of $\left.\mathbb{C} \times\left(\mathbb{C}^{n} \backslash\{0\}\right)\right)$. It was introduced in [3] as a Newton-like operator associated to the evaluation map $F_{A}$, and the following formulas were obtained for $\dot{v}$ and $\dot{\lambda}$ (recall the definition of $A_{\lambda, v}$ from (4)):

$$
\dot{v}=i_{\mathbb{C}^{n}} A_{\lambda, v}{ }^{-1} P_{v^{\perp}} A v, \quad \dot{\lambda}=\frac{\langle(\lambda \mathrm{ld}-A)(v-\dot{v}), v\rangle}{\langle v, v\rangle} .
$$

The map $N_{A}$ is defined for every $(\lambda, v) \in \mathbb{C} \times\left(\mathbb{C}^{n} \backslash\{0\}\right)$ such that $A_{\lambda, v}$ is invertible. It is immediate to check that for $k \geq 0$ and $z \in \mathbb{C}$ we have

$$
N_{z A}^{k}\left(\begin{array}{c}
z \lambda \\
v
\end{array}\right)=\left(\begin{array}{cc}
z & 0 \\
0 & 1
\end{array}\right) N_{A}^{k}\left(\begin{array}{l}
\lambda \\
v
\end{array}\right)
$$

where the superscript ${ }^{k}$ means $k$ iterations. See [3, Sec. 4] for more details.

The notion of approximate solution as a point where Newton's method converges to a true solution immediately and quadratically fast was introduced by Steve Smale [50]. It al- 
lows one to elegantly talk about approximations without dependencies on pre-established accuracies. In addition, these approximate solutions are "excellent approximations" (as mentioned in the statement of the main results) in a very strong sense: the distance to the exact solution dramatically decreases with a single iteration of Newton's method. In the context of eigenpair computations this concept is settled as follows.

Definition 2.11. Given $(A, \lambda, v) \in \mathcal{W}$ we say that $(\zeta, w) \in \mathbb{C} \times\left(\mathbb{C}^{n} \backslash\{0\}\right)$ is an approximate eigenpair of $A$ with associated eigenpair $(\lambda, v)$ when for all $k \geq 1$ the $k$ th iterate $N_{A}^{k}(\zeta, w)$ of the Newton map at $(\zeta, w)$ is well defined and satisfies

$$
\operatorname{dist}_{A}\left(N_{A}^{k}(\zeta, w),(\lambda, v)\right) \leq(1 / 2)^{2^{k}-1} \operatorname{dist}_{A}((\zeta, w),(\lambda, v))
$$

The following result estimates, in terms of the condition of an eigenpair, the radius of a ball of approximate eigenpairs associated to it. For a complete proof see [3, Theorem 5].

Theorem 2.12. There is a universal constant $c_{0}>1 / 5$ with the following property. Let $(A, \lambda, v) \in \mathcal{W}$ with $\|A\|_{F}=1$ and let $(\zeta, w) \in \mathbb{C} \times\left(\mathbb{C}^{n} \backslash\{0\}\right)$. If

$$
\operatorname{dist}_{A}((\lambda, v),(\zeta, w)) \leq \frac{c_{0}}{\mu(A, \lambda, v)},
$$

then $(\zeta, w)$ is an approximate eigenpair of $A$ with associated eigenpair $(\lambda, v)$.

It is a simple exercise to check that for any nonzero $z \in \mathbb{C},(\zeta, w)$ is an approximate zero of $A$ with associated zero $(\lambda, v)$ if and only if $(z \zeta, w)$ is an approximate zero of $z A$ with associated zero $(z \lambda, v)$. So from the point of view of analyzing the effect of the Newton methods we may pick whatever scaling is convenient. For us it will be convenient to assume that $\|A\|_{F}=1$, which we will do in the following.

Proof of Theorem 2.12. Note that [3, Theorem 5] is the same result with $c_{0}=0.2881$, but the definition of the condition number in [3] is slightly different from ours. More exactly, if we denote by $\kappa(A, \lambda, v)$ the condition number defined in [3] then we have $\kappa(A, \lambda, v)=\max (1, \mu(A, \lambda, v))$. Theorem 2.12 is hence true with $\kappa$ in place of $\mu$ and $c_{0}=0.2881$. However, from Lemma 2.6 we know that $\mu(A, \lambda, v) \geq 2^{-1 / 2}$, which readily implies $\kappa(A, \lambda, v) \leq \sqrt{2} \mu(A, \lambda, v)$. Theorem 2.12 now follows from the fact that $0.2881>\sqrt{2} / 5$.

Remark 2.13. We note that $N_{A}(\zeta, w)$ can be computed from the matrix $A$ and the pair $(\zeta, w)$ in $\mathcal{O}\left(n^{3}\right)$ operations, since the cost of this computation is dominated by that of inverting a matrix (or simply solving a linear system).

\subsection{Gaussian measures on $\mathbb{C}^{n \times n}$}

Let $\sigma>0$. We say that the complex random variable $Z=X+\sqrt{-1} Y$ has distribution $\mathcal{N}_{\mathbb{C}}\left(0, \sigma^{2}\right)$ when the real part $X$ and the imaginary part $Y$ are independent and identically distributed (i.i.d.) drawn from $\mathcal{N}\left(0, \sigma^{2} / 2\right)$, i.e., they are Gaussian centered random variables with variance $\sigma^{2} / 2$. 
If $Z \sim \mathcal{N}_{\mathbb{C}}\left(0, \sigma^{2}\right)$ then its density $\varphi: \mathbb{C} \rightarrow \mathbb{R}$ with respect to the Lebesgue measure is given by

$$
\varphi(z):=\frac{1}{\pi \sigma^{2}} e^{-|z|^{2} / \sigma^{2}} .
$$

We will write $v \sim \mathcal{N}_{\mathbb{C}^{n}}\left(0, \sigma^{2}\right)$ to indicate that the vector $v \in \mathbb{C}^{n}$ is random with i.i.d. coordinates drawn from $\mathcal{N}_{\mathbb{C}}\left(0, \sigma^{2}\right)$. Also, we say that $A \in \mathbb{C}^{m \times n}$ is (isotropic) Gaussian and we write $A \sim \mathcal{N}_{\mathbb{C}^{m \times n}}\left(0, \sigma^{2}\right)$ if its entries are i.i.d. Gaussian random variables. The resulting probability space is sometimes called the Ginibre ensemble.

If $\hat{A} \in \mathbb{C}^{m \times n}$ and $G \sim \mathcal{N}_{\mathbb{C}^{m \times n}}\left(0, \sigma^{2}\right)$, we say that the random matrix $A=G+\hat{A}$ has the Gaussian distribution centered at $\hat{A}$, and we write $A \sim \mathcal{N}_{\mathbb{C}^{m \times n}}\left(\hat{A}, \sigma^{2}\right)$. The density of this distribution is given by

$$
\varphi_{m \times n}^{\hat{A}, \sigma}(A):=\frac{1}{\left(\pi \sigma^{2}\right)^{m n}} e^{-\|A-\hat{A}\|_{F}^{2} / \sigma^{2}} .
$$

For conciseness, we will sometimes write $A \sim \mathcal{N}_{\mathbb{C}^{m \times n}}$ when $\hat{A}=0$ and $\sigma=1$.

Crucial in our development is the following result giving a bound on the average condition for Gaussian matrices arbitrarily centered. Its statement is similar to the main technical result in [19, Thm. 3.6]. We will prove it in $\S 7$.

For technical reasons we will be interested in the following variation of $\mu$ :

$$
\mu_{F}(A, \lambda, v):=\|A\|_{F}\left\|A_{\lambda, v}^{-1}\right\|_{F}
$$

(note that we have only replaced $\left\|A_{\lambda, v}^{-1}\right\|$ by $\left\|A_{\lambda, v}^{-1}\right\|_{F}$ ) and the corresponding

$$
\mu_{F, \mathrm{av}}(A):=\left(\frac{1}{n} \sum_{j=1}^{n} \mu_{F}\left(A, \lambda_{j}, v_{j}\right)^{2}\right)^{1 / 2} .
$$

Theorem 2.14. For $\hat{A} \in \mathbb{C}^{n \times n}$ and $\sigma>0$ we have

$$
\underset{A \sim \mathcal{N}_{\mathbb{C}^{n \times n}}\left(\hat{A}, \sigma^{2}\right)}{\mathbb{E}} \frac{\mu_{F, \text { av }}(A)^{2}}{\|A\|_{F}^{2}} \leq \frac{n}{\sigma^{2}} .
$$

Moreover, for A chosen with the uniform distribution $\mathscr{U}(\mathbb{S})$ in the unit sphere $\mathbb{S}$ of $\mathbb{C}^{n \times n}$ we have

$$
\underset{A \sim \stackrel{U}{\mathscr{U}(\mathbb{S})}}{\mathbb{E}} \mu_{F, \text { av }}(A)^{2} \leq n^{3}
$$

Remark 2.15. (i) We note that no bound on the norm of $\hat{A}$ is required in the first claim of Theorem 2.14. Indeed, using $\mu_{F, \text { av }}(s A)=\mu_{F, \text { av }}(A)$, it is easy to see that the assertion for a pair $(\hat{A}, \sigma)$ implies the assertion for $(s \hat{A}, s \sigma)$, for any $s>0$.

(ii) It is remarkable that if we change Gaussian matrices to some classes of structured matrices, the expected value of the condition number can be very high: see for example [10] and references therein. 


\subsection{Truncated Gaussians and smoothed analysis}

For $T, \sigma>0$, we define the truncated Gaussian $\mathcal{N}_{\mathbb{C}^{n \times n}, T}\left(0, \sigma^{2}\right)$ on $\mathbb{C}^{n \times n}$ to be the distribution given by the density

$$
\rho_{T}^{\sigma}(A)= \begin{cases}\varphi_{n \times n}^{0, \sigma}(A) / P_{T, \sigma} & \text { if }\|A\|_{F} \leq T, \\ 0 & \text { otherwise, }\end{cases}
$$

where $P_{T, \sigma}:=\operatorname{Prob}_{A \sim \mathcal{N}_{\mathbb{C}^{n \times n}\left(0, \sigma^{2}\right)}}\left\{\|A\|_{F} \leq T\right\}$, and, we recall, $\varphi_{n \times n}^{0, \sigma}$ is the density of $\mathcal{N}_{\mathbb{C}^{n \times n}}\left(0, \sigma^{2}\right)$. For the rest of this paper we fix the threshold $T:=\sqrt{2} n$. The fact that $\|A\|_{F}^{2}$ is chi-square distributed with $2 n^{2}$ degrees of freedom, along with [20, Corollary 6], yields the following result.

Lemma 2.16. We have $P_{T, \sigma} \geq 1 / 2$ for all $0<\sigma \leq 1$.

The space $\mathbb{C}^{n \times n}$ of matrices with the Frobenius norm and the space $\mathbb{C}^{n^{2}}$ with the canonical Hermitian product are isomorphic as Hermitian product spaces. Hence, the Gaussian $\mathcal{N}_{\mathbb{C}^{n \times n}}\left(0, \sigma^{2}\right)$ on the former corresponds to the Gaussian $\mathcal{N}_{\mathbb{C}^{n^{2}}}\left(0, \sigma^{2}\right)$ on the latter, and we can deduce invariance of $\mathcal{N}_{\mathbb{C}^{n \times n}}\left(0, \sigma^{2}\right)$ under the action of $\mathcal{U}_{n^{2}}$ (in addition to that for conjugation under $\mathcal{U}_{n}$ discussed in $\S 2.3$ ), and the same is true for the truncated Gaussian. In particular, the push-forward of both distributions for the projection $\mathbb{C}^{n \times n} \backslash\{0\} \rightarrow \mathbb{S}$, $A \mapsto A /\|A\|_{F}$, is the uniform distribution $\mathscr{U}(\mathbb{S})$ (see [19, Chapter 2] for details), and

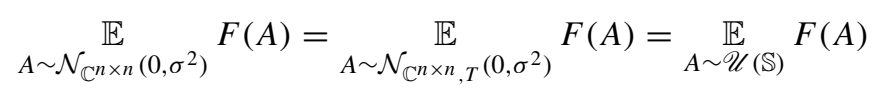

for any measurable scale invariant function $F: \mathbb{C}^{n \times n} \rightarrow[0, \infty)$.

Complexity analysis has traditionally been carried out either in the worst-case or in an average-case. More generally, for a function $F: \mathbb{R}^{m} \rightarrow \mathbb{R}_{+}$(some measure for the computational cost of solving an instance in $\mathbb{R}^{m}$ ), the former amounts to the evaluation of $\sup _{a \in \mathbb{R}^{m}} F(a)$ and the latter to that of $\mathbb{E}_{a \sim \mathscr{D}} F(a)$ for some probability distribution $\mathscr{D}$ on $\mathbb{R}^{m}$. Usually, $\mathscr{D}$ is taken to be the standard Gaussian in the input space. With the beginning of the century, Daniel Spielman and Shang-Hua Teng introduced a third form of analysis, smoothed analysis (see [53, 55] or [19, §2.2.7]), which is meant to interpolate between worst-case and average-case.

The idea is to replace the two operators above (supremum and expectation) by a combination of the two, namely,

$$
\sup _{\hat{a} \in \mathbb{R}^{m}} \underset{a \sim \mathscr{D}(\hat{a}, \sigma)}{\mathbb{E}} F(a)
$$

where $\mathscr{D}(\hat{a}, \sigma)$ is a distribution "centered" at $\hat{a}$ having $\sigma$ as a measure of dispersion. A typical example is the Gaussian $\mathcal{N}\left(\hat{a}, \sigma^{2}\right)$. Another example, used for scale invariant functions $F$, is the uniform measure on a spherical cap centered at $\hat{a}$ and with angular radius $\sigma$ on the unit sphere $\mathbb{S}\left(\mathbb{R}^{m}\right)$ (reference [19] exhibits smoothed analyses for both examples of distribution). In this paper we will perform a smoothed analysis with respect to a truncated Gaussian. More precisely, we will be interested in quantities of the form

$$
\sup _{\hat{A} \in \mathbb{C}^{n \times n}} \underset{A \sim \mathcal{N}_{\mathbb{C}^{n \times n}, T}\left(\hat{A}, \sigma^{2}\right)}{\mathbb{E}} F(A)
$$


where $F$ will be a measure of computational cost for the eigenpair problem. We note that, in addition to the usual dependence on $n$, this quantity depends also on $\sigma$ and tends to $\infty$ when $\sigma$ tends to 0 . When $F$ is scale invariant, as in the case of $\mu_{\mathrm{av}}$ or $\mu_{\max }$, it is customary to restrict attention to matrices of norm 1 , that is, to study the following quantity:

$$
\sup _{\hat{A} \in \mathbb{S}} \underset{A \sim \mathcal{N}_{\mathbb{C}^{n \times n}, T}\left(\hat{A}, \sigma^{2}\right)}{\mathbb{E}} F(A) .
$$

\subsection{The eigenpair continuation algorithm}

We are ready to describe the main algorithmic construct in this paper. When dealing with algorithms it will be more convenient to view the solution variety as the corresponding subset of $\mathbb{C}^{n \times n} \times \mathbb{C} \times\left(\mathbb{C}^{n} \backslash\{0\}\right)$, which, abusing notation, we still denote by $\mathcal{V}$.

Given two matrices $B_{0}, B \in \mathbb{S}, B \neq \pm B_{0}$, let $\alpha:=d_{\mathbb{S}}\left(B_{0}, B\right) \in(0, \pi)$ be the spherical distance (i.e. the angle) from $B_{0}$ to $B$, and let

$$
\mathcal{L}_{B_{0}, B}=\left\{B_{s}: 0 \leq s \leq \alpha\right\}
$$

be the portion of the great circle in $\mathbb{S}$, parametrized by arc-length, joining $B_{0}$ and $B$, so $B_{\alpha}=B$. By abuse of notation, for any $A_{0}, A \in \mathbb{C}^{n \times n}$ such that $A_{0}, A$ are not $\mathbb{R}$-linearly dependent, we simply write

$$
d_{\mathbb{S}}\left(A_{0}, A\right):=d_{\mathbb{S}}\left(A_{0} /\left\|A_{0}\right\|_{F}, A /\|A\|_{F}\right) \quad \text { and } \quad \mathcal{L}_{A_{0}, A}:=\mathcal{L}_{A_{0} /\left\|A_{0}\right\|_{F}, A /\|A\|_{F}} .
$$

The following definition plays a distinguished role in the continuation algorithm. It uses a constant $c_{*}$ which we will later take to be $10^{-4}$ (cf. $\left.\$ 5.1\right)$.

Definition 2.17. If $(A, \lambda, v) \in \mathcal{W},(\zeta, w) \in \mathbb{C} \times\left(\mathbb{C}^{n} \backslash\{0\}\right)$ with $\operatorname{dist}_{A}((\zeta, w),(\lambda, v)) \leq$ $\left.c_{*} / \mu_{(} A, \lambda, v\right)$, then we say that $(\zeta, w)$ is a certified approximate eigenpair of $A$ (with associated eigenpair $(\lambda, v)$ ). The initial neighborhood of the set $\mathcal{W}$ is the set

$$
\tilde{\mathcal{W}}:=\left\{(A, \zeta, w) \mid \exists(\lambda, v):(A, \lambda, v) \in \mathcal{W} \text { and } \operatorname{dist}_{A}((\zeta, w),(\lambda, v)) \leq \frac{c_{*}}{\mu_{\max }(A)}\right\} .
$$

The term certified in Definition 2.17 is justified by Theorem 2.12.

Suppose that we are given an initial triple $\left(B_{0}, \zeta_{0}, w_{0}\right) \in \tilde{\mathcal{W}}, B_{0} \in \mathbb{S}$ and an input matrix $B \in \mathbb{S} \backslash\left\{ \pm B_{0}\right\}$. Let $\left(\lambda_{0}, v_{0}\right) \in \mathbb{C} \times \mathbb{P}\left(\mathbb{C}^{n}\right)$ be the exact eigenpair of $B_{0}$ associated to $\left(\zeta_{0}, v_{0}\right)$. As a consequence of the inverse function theorem, if $\left(\mathcal{L}_{B_{0}, B} \backslash\left\{B_{0}\right\}\right) \cap \Sigma=\emptyset$, then the map $s \mapsto B_{s}$ can be uniquely extended to a continuous map

$$
[0, \alpha] \rightarrow \mathcal{V}, \quad s \mapsto\left(B_{s}, \lambda_{s}, v_{s}\right) .
$$

We call this map the lifting of $\mathcal{L}_{B_{0}, B}$ with origin $\left(B_{0}, \lambda_{0}, v_{0}\right)$. We can try to approximate the eigenpair $\left(\lambda_{\alpha}, v_{\alpha}\right)$ of $B$ by following the lifting of $\mathcal{L}_{B_{0}, B}$. To this end we can differentiate $B_{s} v_{s}-\lambda_{s} v_{s}=0$ with respect to $s$. This produces an Initial Value Problem (IVP) whose solution can be approximated by any standard numerical ODE solver. The main ingredient for the complexity estimate is the number of points in the discretization of $[0, \alpha]$ needed to approximate the solution of the IVP.

Formalizing this idea to get an actual guarantee of convergence is a nontrivial task; only a nonconstructive method has been described in [3] following the ideas in [44]. We now describe how to algorithmically construct a numerically stable method for this task: 


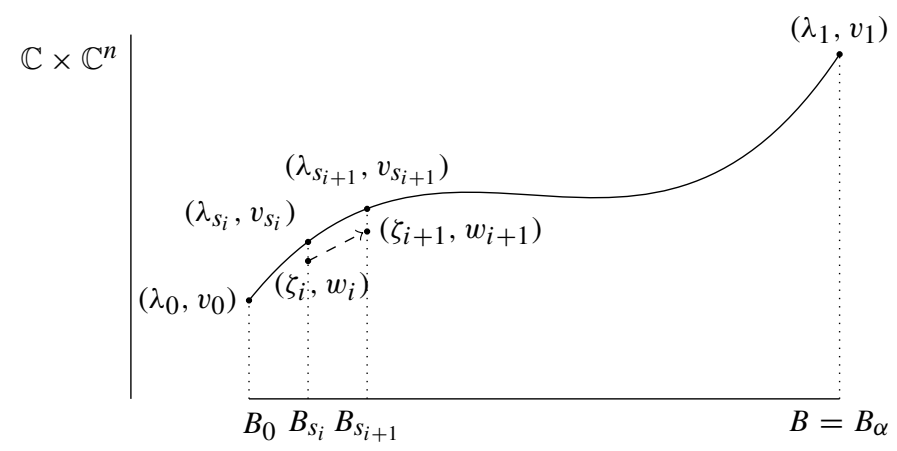

Fig. 1. The continuation of the solution path.

subdivide the interval $[0, \alpha]$ into subintervals with extremities at $0=s_{0}<s_{1}<\cdots<s_{K}$ $=\alpha$ and successively compute approximations $\left(\zeta_{i}, w_{i}\right)$ of $\left(\lambda_{s_{i}}, v_{s_{i}}\right)$, starting with $\left(\zeta_{0}, w_{0}\right)$ and then using Newton's method. To ensure that these are good approximations, we actually want to ensure that for all $i,\left(\zeta_{i}, w_{i}\right)$ is an approximate eigenpair of $B_{s_{i+1}}$. Figure 1 attempts to convey the general idea.

The algorithmic counterpart of this idea is the following.

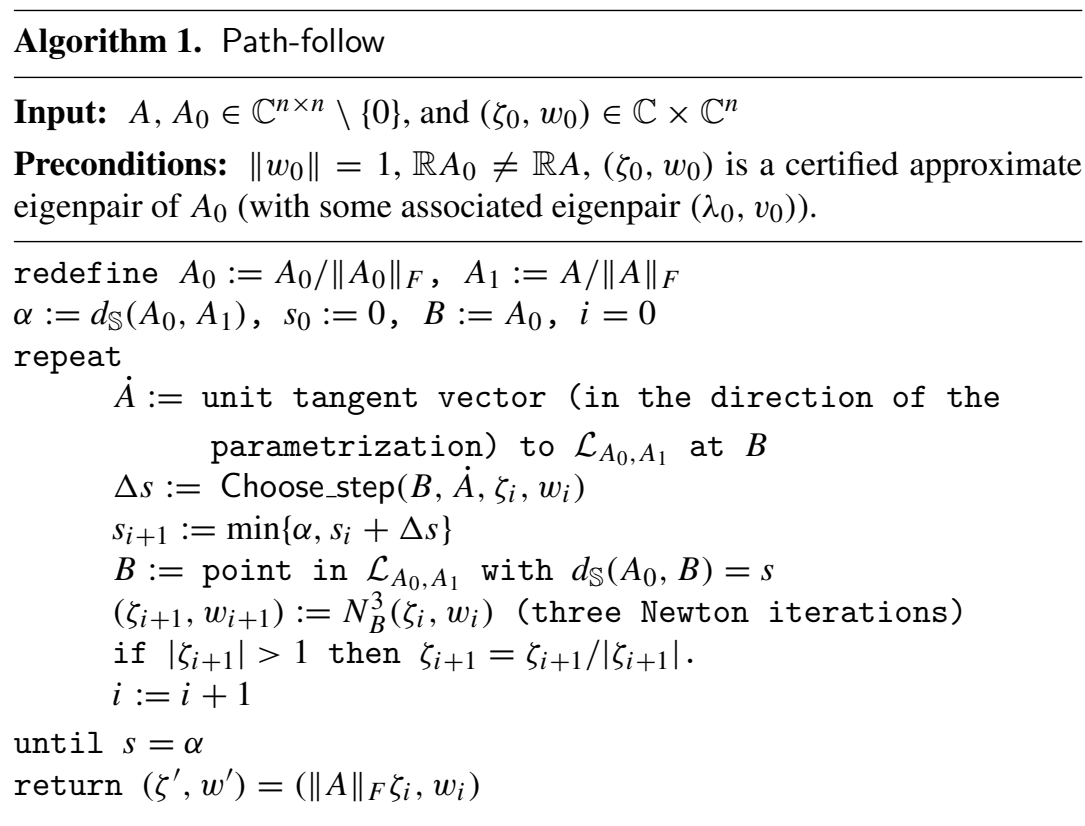

Output: $\left(\zeta^{\prime}, w^{\prime}\right) \in \mathbb{C} \times \mathbb{C}^{n}$

Postconditions: The execution halts if the lifting $\left(A_{t}, \lambda_{t}, v_{t}\right), 0 \leq t \leq$ $d_{\mathbb{S}}\left(A_{0}, A\right)$, of $\mathcal{L}_{A_{0}, A}$ at $\left(\lambda_{0}, v_{0}\right)$ does not meet $\Sigma^{\prime}$. In this case, $\left(\zeta^{\prime}, \overline{w^{\prime}}\right)$ is a certified approximate eigenpair of $A$ with associated eigenpair $\left(\lambda_{1}, v_{1}\right)$. 
Remark 2.18. Note that in particular any triple $\left(A_{0}, \lambda_{0}, v_{0}\right) \in \mathcal{W}$ is an acceptable starting point for Path-follow for generic $A \in \mathbb{C}^{n \times n}$.

The algorithm Path-follow is unambiguous except for the subroutine Choose_step, which will be described at the end of $\$ 5.2$. We next state the main results for it. Recall that the point $s_{\ell} \in[0, \alpha]$ is the value of $s$ generated by Path-follow at the $\ell$ th iteration. For $K \in \mathbb{R}$ we denote by $\lceil K\rceil$ the smallest integer which is greater than or equal to $K$.

Theorem 2.19. Suppose that $\mathcal{L}_{A_{0}, A}=\left\{B_{s}\right\}_{s \in[0, \alpha]}$ (so $B_{0}=A_{0} /\left\|A_{0}\right\|_{F}$ and $B_{\alpha}=$ $A /\|A\|_{F}$ ) and assume that $\mathcal{L}_{A_{0}, A} \cap \Sigma=\emptyset$. Then the algorithm Path-follow stops after at most $\lceil K\rceil$ steps where $K$ is given by

$$
K:=K\left(A, A_{0}, \zeta_{0}, w_{0}\right):=C \int_{0}^{\alpha} \mu\left(B_{s}, \lambda_{s}, v_{s}\right)\left\|\left(\dot{B}_{s}, \dot{\lambda}_{s}, \dot{v}_{s}\right)\right\| d s .
$$

Here the pairs $\left(\lambda_{s}, v_{s}\right)$ are given by (17).

More generally, let $q \in \mathbb{Z}, q \geq 0$. Then Path-follow stops after at most (the smallest integer greater than or equal to)

$$
q+C \int_{s_{q}}^{\alpha} \mu\left(B_{s}, \lambda_{s}, v_{s}\right)\left\|\left(\dot{B}_{s}, \dot{\lambda}_{s}, \dot{v}_{s}\right)\right\| d s
$$

steps. The returned pair $(\zeta, w)$ is an approximate eigenpair of $A$ with associated eigenpair $\left(\lambda_{\alpha}, v_{\alpha}\right)$. Here, $C \leq 3000$ is a universal constant.

For $0 \leq a<b \leq \alpha$, the quantity

$$
L_{\mu, a, b}\left(B_{s}, \lambda_{s}, v_{s}\right)=\int_{a}^{b} \mu\left(B_{s}, \lambda_{s}, v_{s}\right)\left\|\left(\dot{B}_{s}, \dot{\lambda}_{s}, \dot{v}_{s}\right)\right\| d s
$$

in Theorem 2.19 is the length of the curve $\left\{\left(B_{s}, \lambda_{s}, v_{s}\right): a \leq s \leq b\right\}$ in the so-called condition metric. This is the metric that is obtained by pointwise multiplying the natural metric in $\mathbb{S} \times \mathbb{C} \times \mathbb{P}\left(\mathbb{C}^{n}\right)$ by the condition number squared. We call $L_{\mu, a, b}\left(B_{s}, \lambda_{s}, v_{s}\right)$ the condition length of this curve.

The proof of Theorem 2.19 is given in $\$ 5.3$.

Remark 2.20. From Proposition 2.7 , we have

$$
L_{\mu, s_{q}, \alpha}\left(B_{s}, \lambda_{s}, v_{s}\right) \leq \sqrt{6} \int_{s_{q}}^{\alpha} \mu\left(B_{s}, \lambda_{s}, v_{s}\right)^{2} d s .
$$

The following result gives an alternative bound to the number of steps.

Corollary 2.21. Let $A_{0}, A \in \mathbb{C}^{n \times n}$ be $\mathbb{R}$-linearly independent and consider the path $A_{t}=(1-t) A_{0}+t A$ which satisfies $A_{1}=A$. If $\mathcal{L}_{A_{0}, A} \cap \Sigma=\emptyset$ then, for any $q \in \mathbb{Z}, q \geq 0$, the algorithm Path-follow stops after at most $\lceil K\rceil$ steps where

$$
K:=q+\sqrt{6} C\left\|A_{0}\right\|_{F}\left\|A_{1}\right\|_{F} \int_{t_{q}}^{1} \frac{\mu\left(A_{t}, \lambda_{t}, v_{t}\right)^{2}}{\left\|A_{t}\right\|_{F}^{2}} d t
$$


with

$$
t_{q}:=\frac{\left\|A_{0}\right\|_{F}}{\left\|A_{1}\right\|_{F}\left(\sin \alpha \cot s_{q}-\cos \alpha\right)+\left\|A_{0}\right\|_{F}} .
$$

Here, $\lambda_{t}, v_{t}$ are the eigenvalue and eigenvector of $A_{t}$ defined by continuation.

Proof. From Theorem 2.19 and Remark 2.20, if we let $C^{\prime}=\sqrt{6} C$, the number of steps is at most the smallest integer greater than or equal to

$$
q+C^{\prime} \int_{s_{q}}^{\alpha} \mu\left(B_{s}, \lambda_{B_{s}}, v_{B_{s}}\right)^{2} d s=q+C^{\prime} \int_{s_{q}}^{\alpha} \mu\left(B_{s}, \lambda_{B_{s}}, v_{B_{s}}\right)^{2}\left\|\dot{B}_{s}\right\|_{F} d s,
$$

where $\lambda_{B_{s}}$ and $v_{B_{s}}$ are the eigenvalue and eigenvector of $B_{S}$ defined by continuation. Now, if we reparametrize that spherical segment by $C_{t}=A_{t} /\left\|A_{t}\right\|_{F}, t \in[0,1]$, the integral does not change. The quantity above is thus equal to

$q+C^{\prime} \int_{t_{q}}^{1} \mu\left(C_{t}, \lambda_{C_{t}}, v_{C_{t}}\right)^{2}\left\|\dot{C}_{t}\right\|_{F} d t \underset{\text { Rmk. } 2.5}{=} q+C^{\prime} \int_{t_{q}}^{1} \mu\left(A_{t}, \lambda_{t}, v_{t}\right)^{2}\left\|\frac{d}{d t}\left(\frac{A_{t}}{\left\|A_{t}\right\|_{F}}\right)\right\| d t$.

Substituting $\dot{A}_{t}=A_{1}-A_{0}$ and $A_{t}=(1-t) A_{0}+t A_{1}$ in this last formula and with some elementary computations (see [19, Lemma 17.5]) we conclude that

$$
\left\|\frac{d}{d t}\left(\frac{A_{t}}{\left\|A_{t}\right\|_{F}}\right)\right\| \leq \frac{\left\|A_{0}\right\|_{F}\left\|A_{1}\right\|_{F}}{\left\|A_{t}\right\|_{F}^{2}} .
$$

The corollary follows (the value of $t_{q}$ is obtained by the reparametrization from $s_{q}$ ).

The inequality of Remark 2.20 implies that (up to constants) the upper bound for the number of steps by an algorithm in terms of the condition length as in Theorem 2.19 is smaller than the upper bound in terms of the integral of the squared condition number as in Corollary 2.21. A similar situation applies in the context of polynomial system root finding. In this case implementations exist in both contexts (see [49, 18, 11, 23]). The condition length algorithm is more subtle and the proof of correctness more difficult, both for the polynomial system and eigenpair cases. So the temptation is to present condition number squared algorithms. Here for the first time we present a quantitative estimate of the improvement which is significant and which justifies presenting the more complex condition length algorithm (another estimate of this kind was established shortly after in [4]). In Theorem 2.29 a randomized algorithm is studied. The upper bound given by the condition length algorithm is $O\left(n^{2}\right)$ while an algorithm with complexity given by the condition number squared would give $O\left(n^{3}\right)$.

In our main results we are interested in the cost of our algorithms over random matrices $A$. The following quantity-the expected number of iterations of Path-follow for a given initial triple $\left(A_{0}, \lambda_{0}, v_{0}\right) \in \mathcal{W}$-becomes essential:

$$
\text { Avg_Num_Iter }\left(A_{0}, \lambda_{0}, v_{0}\right):=\underset{A \sim \mathcal{N}_{\mathbb{C}^{n \times n}}}{\mathbb{E}} K\left(A, A_{0}, \lambda_{0}, v_{0}\right)
$$


We can also consider the smoothed number of iterations of Path-follow that results by drawing instead the input matrix $A$ from $\mathcal{N}_{T}\left(\hat{A}, \sigma^{2}\right)$ where $\hat{A} \in \mathbb{S}$ is arbitrary. We thus define

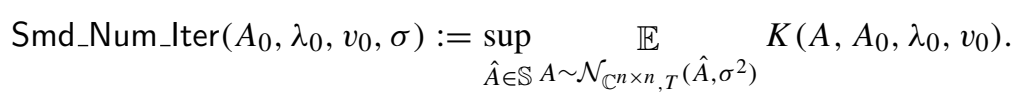

Proposition 2.22. For $\left(A_{0}, \lambda_{0}, v_{0}\right) \in \mathcal{W}$ we have

$$
\operatorname{Avg} \_ \text {Num_Iter }\left(A_{0}, \lambda_{0}, v_{0}\right)=\mathcal{O}\left(n^{4} \mu\left(A_{0}, \lambda_{0}, v_{0}\right)^{2}\right)
$$

and, for all $\sigma \in(0,1]$,

$$
\text { Smd_Num_Iter }\left(A_{0}, \lambda_{0}, v_{0}, \sigma\right)=\mathcal{O}\left(n^{4} \mu\left(A_{0}, \lambda_{0}, v_{0}\right)^{2} / \sigma^{2}\right) .
$$

Remark 2.23. Note that Proposition 2.22 ensures that Path-follow halts in finite time with probability 1 even if $A_{0} \in \Sigma$, as long as $\mu\left(A_{0}, \lambda_{0}, v_{0}\right)$ is finite. The algorithm does a first step that depends on $\mu\left(A_{0}, \lambda_{0}, v_{0}\right)$ only and advances to $B_{s_{1}}$ with $s_{1}>0$. After that, the fact that the real codimension of $\Sigma$ in $\mathbb{C}^{n \times n}$ is 2 (shown in Proposition 2.3) ensures that, almost surely, $\left(\mathcal{L}_{A_{0}, A} \backslash\left\{A_{0}\right\}\right) \cap \Sigma=\emptyset$. Therefore, with probability 1 , none of the matrices $B_{S}$ is in $\Sigma$ and the integral over $\left[s_{1}, \alpha\right]$ in Theorem 2.19 is finite. Also, note that one does not need to have the exact eigenpair $\left(\lambda_{0}, v_{0}\right)$, but it suffices to have a certified approximate eigenpair of $A_{0}$ with associated eigenpair $\left(\lambda_{0}, v_{0}\right)$. In particular, it suffices to have $\left(A_{0}, \lambda_{0}, w_{0}\right) \in \tilde{\mathcal{W}}$.

To compute all the eigenpairs from an initial matrix $A_{0} \in \mathbb{C}^{n \times n} \backslash \Sigma$ and its eigenpairs $\left(\lambda^{(1)}, v^{(1)}\right), \ldots,\left(\lambda^{(n)}, v^{(n)}\right)$ we may proceed by following the $n$ paths corresponding to taking these eigenpairs in the initial triples. In this case, we will be interested in the quantities

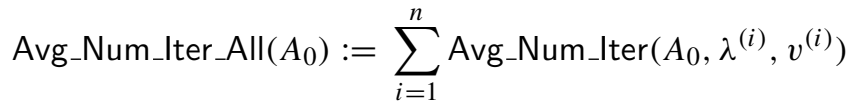

$$
\begin{aligned}
& =\underset{A \sim \mathcal{N}_{\mathbb{C}^{n \times n}}}{\mathbb{E}} \sum_{i=1}^{n} K\left(A, A_{0}, \lambda^{(i)}, v^{(i)}\right)
\end{aligned}
$$

and

$$
\text { Smd_Num_Iter_All }\left(A_{0}, \sigma\right):=\sup _{\hat{A} \in \mathbb{S}} \mathbb{A \sim \mathcal { N } _ { \mathbb { C } ^ { n \times n } , T } ( \hat { A } , \sigma ^ { 2 } )} \underset{i=1}{\mathbb{E}} K\left(A, A_{0}, \lambda^{(i)}, v^{(i)}\right) .
$$

For these quantities we prove the following result.

Proposition 2.24. For $A_{0} \in \mathbb{C}^{n \times n} \backslash \Sigma$ we have

$$
\text { Avg_Num_Iter_All }\left(A_{0}\right)=\mathcal{O}\left(n^{4} \mu_{\max }\left(A_{0}\right)^{2}\right)
$$

and, for all $\sigma \in(0,1]$,

$$
\text { Smd_Num_Iter_All }\left(A_{0}, \sigma\right)=\mathcal{O}\left(n^{4} \mu_{\max }\left(A_{0}\right)^{2} / \sigma^{2}\right) \text {. }
$$


We prove Propositions 2.22 and 2.24 in Section 8. Note that the latter does not immediately follow from the former since in general

$$
\sum_{i=1}^{n} \mu\left(A_{0}, \lambda^{(i)}, v^{(i)}\right)^{2} \gg \mu_{\max }\left(A_{0}\right)^{2} .
$$

\subsection{Initial triples and global algorithms}

The Path-follow routine assumes an initial triple $\left(A_{0}, \lambda_{0}, v_{0}\right)$ at hand. We next deal with this issue. We first consider the case of computing a single eigenpair. In this case we consider the diagonal matrix $H$ whose diagonal entries are $(1,0, \ldots, 0)$ and its wellposed eigenpair $\left(1, e_{1}\right)$.

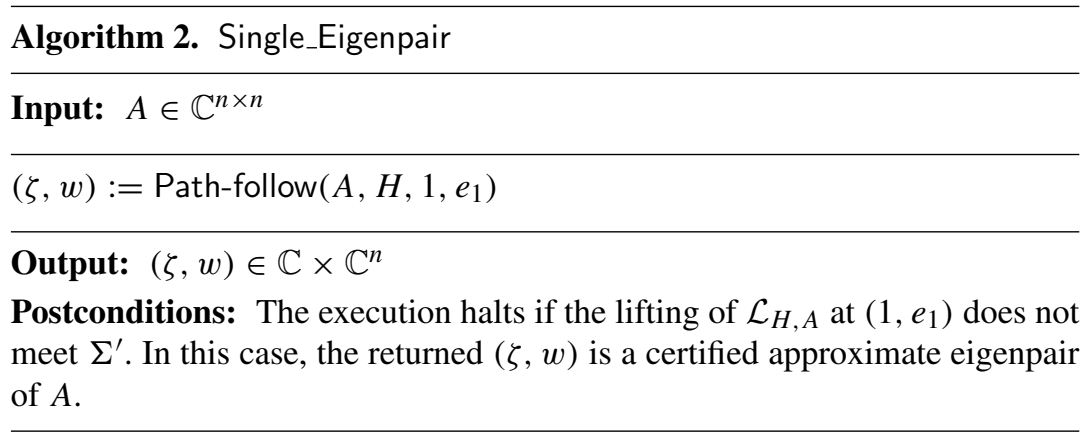

We can formally state (and prove) the first of our main results. To this end, we define the average cost Avg_Cost $(n)$ of Single_Eigenpair to be the average (over the input matrix $A$ ) of the number of arithmetic operations performed by the algorithm. We similarly define its smoothed cost Smd_Cost $(n, \sigma)$.

Theorem 2.25. The algorithm Single_Eigenpair returns (almost surely) an approximate eigenpair of its input $A \in \mathbb{C}^{n \times n}$. Its average cost satisfies

$$
\operatorname{Avg} \_\operatorname{Cost}(n)=\mathcal{O}\left(n^{7}\right) \text {. }
$$

For every $0<\sigma \leq 1$, its smoothed cost satisfies

$$
\text { Smd_Cost }(n, \sigma)=\mathcal{O}\left(n^{7} / \sigma^{2}\right) \text {. }
$$

Proof. Lemma 2.10 and $\|H\|_{F}=1$ imply that $\mu\left(H, 1, e_{1}\right)=1$. The statement is then a consequence of Proposition 2.22 and the fact that the average cost is obtained by multiplying Avg_Num_Iter $\left(H, 1, e_{1}\right)$ by the $\operatorname{cost} \mathcal{O}\left(n^{3}\right)$ of each iteration.

Remark 2.26. The triple $\left(H, 1, e_{1}\right)$ is the version, in our context, of the initial pair proposed by Shub and Smale [49] for the computation of zeros of polynomial systems. In this later context, the problem of showing that one can efficiently follow linear homotopies with this initial pair remains open. 
The fact that any other eigenpair of $H$ is ill-posed prevents us from using them to compute other eigenpairs of $A$. If we want to compute all the eigenpairs of $A$, we will need to consider a different approach.

To do so, for any fixed $n \geq 2$, let

$$
D:=\operatorname{diag}\left(\eta_{1}, \ldots, \eta_{n}\right)
$$

where the $\eta_{i}$ are points in the unit side hexagonal lattice chosen in such an order that $0=\left|\eta_{1}\right| \leq \cdots \leq\left|\eta_{n}\right|$. We recall that the hexagonal lattice is the set of points of the form

$$
Q\left(\begin{array}{l}
a \\
b
\end{array}\right), \quad \text { where } \quad Q:=\left(\begin{array}{cc}
1 & 1 / 2 \\
0 & \sqrt{3} / 2
\end{array}\right), \quad a, b \in \mathbb{Z}
$$

Lemma 2.27. We have $\mu_{\max }(D) \leq \frac{\sqrt{3 / 2}}{\pi} n+o(n)$.

Proof. We first find a real number $r>0$ with the property that the disk $\mathbb{D}(r)$ of radius $r$ contains at least $n$ points in the hexagonal lattice. To do so, note that a lattice point $Q\left(\begin{array}{l}a \\ b\end{array}\right)$ is in $\mathbb{D}(r)$ if and only if

$$
\left(\begin{array}{l}
a \\
b
\end{array}\right) \in Q^{-1} \mathbb{D}(r)
$$

Now, the singular values of $Q^{-1}$ are $\sqrt{2}$ and $\sqrt{2 / 3}$, so $Q^{-1} \mathbb{D}(r)$ is an ellipse of area $2 \pi r^{2} / \sqrt{3}$ and maximal radius $\sqrt{2} r$. Dividing by the smallest integer $N>2 \sqrt{2} r$ and translating the resulting ellipse to have center $(1 / 2,1 / 2)$, we look for points of the form $(a / N, b / N)$ with $a, b \in\{0, \ldots, N-1\}$ which lie inside an ellipse of area

$$
\frac{2 \pi r^{2}}{\sqrt{3} N^{2}}
$$

contained in $[0,1]^{2}$. This is a particular instance of the problem of counting lattice points in semialgebraic sets, a well studied problem for which a quite complete solution is for example [14, Th. 3, p. 327]. We conclude that the number of points in the hexagonal lattice in $\mathbb{D}(r)$ is at least

$$
\frac{2 \pi r^{2}}{\sqrt{3}}-2 N \geq \frac{2 \pi r^{2}}{\sqrt{3}}-4 \sqrt{2} r-2
$$

In particular, we can find $n$ lattice points in a disk of radius $r=3^{1 / 4} /\left((2 \pi)^{1 / 2} n^{1 / 2}+\right.$ $\left.o\left(n^{1 / 2}\right)\right)$. Moreover, around each lattice point we can place a circle of radius $1 / 2$ without overlappings. From the mean value equality for analytic functions, for any $z \in \mathbb{C}$ and $\varepsilon>0$ we have

$$
|z|^{2}=\left|z^{2}\right|=\left|\frac{1}{\pi \varepsilon^{2}} \int_{|y-z|<\varepsilon} y^{2} d y\right| \leq \frac{1}{\pi \varepsilon^{2}} \int_{|y-z|<\varepsilon}|y|^{2} d y .
$$

We thus have

$$
\|D\|_{F}^{2}=\sum_{j=1}^{n}\left|\eta_{j}\right|^{2} \leq \sum_{z}|z|^{2} \leq \frac{4}{\pi} \sum_{z} \int_{|y-z|<1 / 2}|y|^{2} d y \leq \frac{4}{\pi} \int_{|y|<r+1 / 2}|y|^{2} d y,
$$


where $z$ runs over all lattice points contained in $\mathbb{D}(r)$. Computing the last integral yields

$$
\|D\|_{F}^{2} \leq 2 r^{4}+o\left(r^{4}\right)=\frac{3 n^{2}}{2 \pi^{2}}+o\left(n^{2}\right)
$$

Finally, from Lemma 2.10 we conclude that

$$
\mu_{\max }(D)^{2}=\|D\|_{F}^{2} \leq \frac{3 n^{2}}{2 \pi^{2}}+o\left(n^{2}\right) .
$$

We now put together the continuation algorithm Path-follow and this specific initial triple.

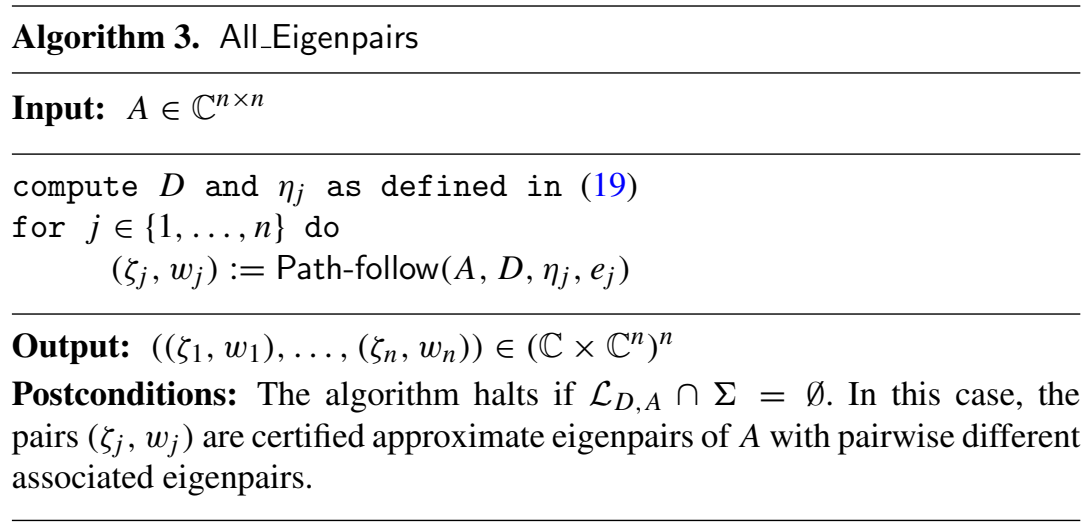

We can now state (and prove) the second of our main results.

Theorem 2.28. The algorithm All_Eigenpairs returns (almost surely) $n$ approximate eigenpairs of its input $A \in \mathbb{C}^{n \times n}$, with pairwise different associate eigenpairs. Its average cost satisfies

$$
\operatorname{Avg} \operatorname{Cost}(n)=\mathcal{O}\left(n^{9}\right) \text {. }
$$

For every $\sigma \leq 1$ its smoothed cost satisfies

$$
\text { Smd_Cost }(n, \sigma)=\mathcal{O}\left(n^{9} / \sigma^{2}\right) \text {. }
$$

Proof. This easily follows from Lemma 2.27 and Proposition 2.24. As in the proof of Theorem 2.25, we recall that the cost of each iteration is $\mathcal{O}\left(n^{3}\right)$.

\subsection{Computer experiments}

The algorithm Single_Eigenpair was implemented in Matlab by Liu Yiting, and this implementation was used to get an empirical estimate of its average number of iterations and average cost.

To estimate these averages she generated 200 random complex matrices $A_{j} \in \mathbb{C}^{n \times n}$ for $n=4,8,16,32$. For each $n$ and each $j$ she computed an eigenpair for $A_{j}$ and recorded the number $I_{n j}$ of iterations performed by Single_Eigenpair to do so. Then she computed 
the (empirical) average number of iterations $\operatorname{Iter}(n)=\frac{1}{200} \sum_{j=1}^{200} I_{n j}$. She did the same for $n=64$ but only with 30 matrices. She obtained, respectively, 1571.4, 3464.9, 6410.4, 9390.6, and 13941.

In order to estimate the growth of Iter $(n)$ with $n$ she did a linear regression between $\log _{2}$ Iter $(n)$ and $\log _{2} n$ for $n=4,8,16,32$ and 64 . The regression line obtained was $\log _{2} \operatorname{Iter}(n) \approx 0.7737\left(\log _{2} n\right)+9.3026$. The value of the regression coefficient, close to 1 , shows that this line is a very good fit to the functional dependence $\log _{2} \operatorname{Iter}(n)=$ $f\left(\log _{2} n\right)$. Taking exponential with base 2 on both sides, we get $\operatorname{Iter}(n) \approx 632 n^{0.78}$, and therefore an empirical estimate of $\mathcal{O}\left(n^{3.78}\right)$ for Avg_Cost $(n)$, substantially better than the $\mathcal{O}\left(n^{7}\right)$ in Theorem 2.25 (but still worse than the empirical behavior of the eigensolvers currently used).

\subsection{Randomized algorithms}

In this section we follow the ideas in [13] adapting them to the case of eigenpair computations. Consider the probability distribution $\mathcal{D}$ in the solution variety $\mathcal{V}$ defined via the following procedure:

$$
\begin{aligned}
& \text { randomly choose } A_{0} \sim \mathcal{N}_{\mathbb{C}^{n \times n}} \\
& \text { randomly choose one eigenpair }\left(\lambda_{0}, v_{0}\right) \text { of } A_{0}
\end{aligned}
$$

Next assume that we have a routine $\operatorname{draw}_{-}$from $\_\mathcal{D}$ to draw triples $\left(A_{0}, \lambda_{0}, v_{0}\right)$ from the distribution $\mathcal{D}$ on $\mathcal{V}$. Then we can consider the following algorithm.

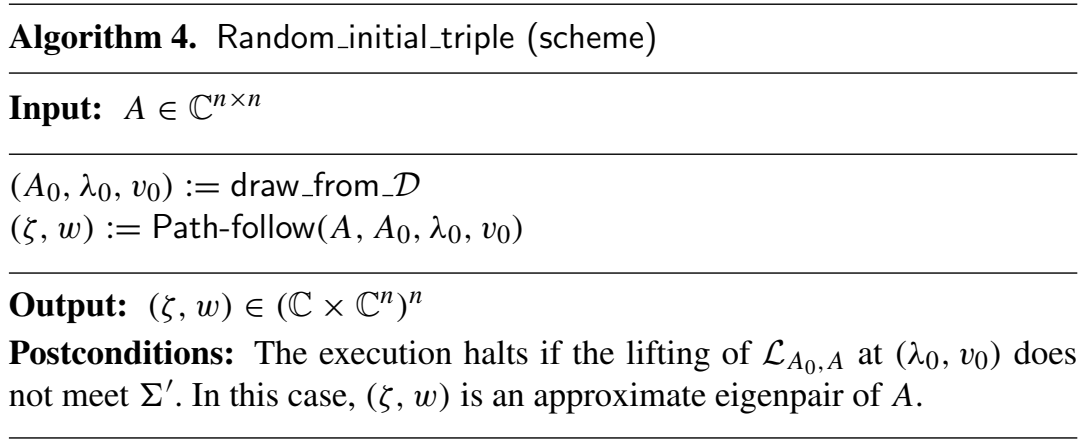

The interest of this algorithmic scheme is that we can prove better bounds (than those in Theorem 2.25) for the number of iterations of Path-follow.

Theorem 2.29. The expected average number of homotopy steps of Path-follow in Algorithm 4 satisfies

$$
\underset{A \sim \mathcal{N}_{\mathbb{C}^{n \times n}}}{\mathbb{E}} \underset{\left(A_{0}, \lambda_{0}, v_{0}\right) \sim \mathcal{D}}{\mathbb{E}} K\left(A, A_{0}, \lambda_{0}, v_{0}\right) \leq 4 C n^{2},
$$

where $C$ is as in Theorem 2.19. 
Theorem 2.29, which will be proved in Section 9, brings to focus the need of an implementation of draw_from_ $\mathcal{D}$. It must be noted though that a direct implementation is not possible since the second line in (20) (choosing $\left(\lambda_{0}, v_{0}\right)$ at random) implicitly requires solving an EVP problem, the very question that this article is attempting to solve! This is a similar situation to that solved in $[12,13]$, where a random polynomial system and one of its zeros at random had to be chosen. It is also similar to that dealt with in [5] for the computation of eigenpairs of Hermitian matrices. A version of the proof of Theorem 2.29 with the Gaussian Unitary Ensemble replacing the Gaussian distribution actually yields an improvement over the main result in [5]. (We note in passing that the case of Hermitian matrices is one of the few instances where a proof of convergence of a practical eigensolver, such as the Francis-Kublanovskaya's QR, is actually known [7].)

It must also be noted that by following the method in [34] for the polynomial system case Algorithm 4 can probably be de-randomized without increasing the complexity.

Corollary 2.30. In the case of Hermitian matrices, the expected average number of homotopy steps of Path-follow in Algorithm 4 (with the randomization algorithm in [5] in place of draw_from_D) is $\mathcal{O}\left(n^{2}\right)$. This yields an expected cost of $\mathcal{O}\left(n^{5}\right)$ for the computation of one eigenpair and of $\mathcal{O}\left(n^{6}\right)$ for the computation of all of them. Here the input matrix $H$ is drawn from the Gaussian Unitary Ensemble.

Following the ideas in those papers, we note that Theorem 2.29 would yield an algorithm with average running time $\mathcal{O}\left(n^{5}\right)$ if we could find some collection of probability spaces $\Omega_{n}$ and functions $\psi_{n}: \Omega_{n} \rightarrow \mathcal{V}_{n}, n \geq 2$, such that:

1. Choosing $\omega \in \Omega_{n}$ can be done starting with a number of random choices of numbers with the $\mathcal{N}_{\mathbb{C}}$ distribution, and performing some arithmetic operations on the results, the total expected running time being at most $\mathcal{O}\left(n^{5}\right)$.

2. Given $\omega \in \Omega_{n}, \psi_{n}(\omega)$ is computable in average time $\mathcal{O}\left(n^{5}\right)$, that is, the expected number of arithmetic operations for computing $\psi_{n}(\omega)$ must be $\mathcal{O}\left(n^{5}\right)$.

3. Choosing $\omega$ at random in $\Omega_{n}$ and computing $\left(A_{0}, \lambda_{0}, v_{0}\right)=\psi_{n}(\omega)$ is equivalent to choosing $A_{0} \sim \mathcal{N}_{\mathbb{C}^{n \times n}}$ at random and choosing at random $\left(\lambda_{0}, v_{0}\right)$ such that $A v_{0}=$ $\lambda_{0} v_{0}$. That is, for any measurable mapping $\phi: \mathcal{V}_{n} \rightarrow[0, \infty]$ we must have

$$
\underset{\omega \sim \Omega_{n}}{\mathbb{E}} \phi\left(\psi_{n}(\omega)\right)=\underset{A_{0} \sim \mathcal{N}_{\mathbb{C}^{n \times n}}^{\mathbb{E}}}{\mathbb{E}} \frac{1}{n} \sum_{\lambda_{0}, v_{0}:} \phi\left(A_{0}, \lambda_{0}, v_{0}\right),
$$

so that we can apply this equality to

$$
\phi\left(A_{0}, \lambda_{0}, v_{0}\right)=\underset{A \sim \mathcal{N}_{\mathbb{C}^{n \times n}}}{\mathbb{E}} K\left(A, A_{0}, \lambda_{0}, v_{0}\right)
$$

and apply Theorem 2.29.

Unfortunately, we are not able to produce a collection of probability spaces $\Omega_{n}$ and functions $\psi_{n}$ as described above. However, we will prove that relaxing (21) to the following less restrictive situation is actually possible: instead of demanding the equality in (21) we 
can just demand an inequality where the right-hand term is multiplied by some polynomial in $n$. Moreover, we do not need (21) to hold for every measurable function $\phi$ since all the interesting functions for the EVP problem are projective functions, invariant under the action of the unitary group. We can thus relax (21) to hold only with a polynomially bounded inequality, and for unitary invariant projective functions. Proving that this can actually be done is our goal now. (Meanwhile our ideas have been adapted to the more general setting of eigenpairs of polynomial systems in [15].)

We start by defining $\Omega_{n}$ and $\psi_{n}$. Consider the classical Stiefel manifold consisting of orthonormal $(n-1)$-frames in $\mathbb{C}^{n}$, given by

$$
\mathcal{S}_{n-1}\left(\mathbb{C}^{n}\right):=\left\{Q \in \mathbb{C}^{n \times(n-1)}: Q^{*} Q=I_{n-1}\right\},
$$

endowed with its natural probability measure given by the restriction of the Frobenius Hermitian structure to the tangent bundle.

For every $n \geq 2$, let

$$
\mathcal{A}_{n}:=\left\{(M, Q): \operatorname{ker}(M)=\operatorname{ker}\left(Q^{*}\right)\right\} \subseteq \mathbb{C}^{(n-1) \times n} \times \mathcal{S}_{n-1}\left(\mathbb{C}^{n}\right) .
$$

In other words, $\mathcal{A}_{n}$ consists of pairs of matrices $M, Q$ such that the columns of $Q$ form an orthonormal basis of the complement of $\operatorname{ker}(M)$. The set $\mathcal{A}_{n}$ has a natural probability measure $\mu_{\mathcal{A}_{n}}$ given by

$$
\mu_{\mathcal{A}_{n}}(X):=\underset{M \sim \mathcal{N}_{\mathbb{C}^{(n-1) \times n}}}{\mathbb{E}} \underset{(M, Q) \in \mathcal{A}_{n}}{\mathbb{E}} \mathbb{1}_{X}(M, Q)
$$

for measurable sets $X \subseteq \mathcal{A}_{n}$. Here, $Q$ has the uniform distribution in the compact manifold $\left\{Q \in \mathcal{S}_{n-1}\left(\mathbb{C}^{n}\right):(M, Q) \in \mathcal{A}_{n}\right\}$ and $\mathbb{1}_{X}$ is the indicator function of $X$.

Definition 2.31. Let

$$
\Omega_{n}:=\left\{(\lambda, w,(M, Q)): 2 \Re(\bar{\lambda} \operatorname{tr}(M Q)) \leq 1-|\lambda|^{2}(n-1)\right\} \subseteq \mathbb{C} \times \mathbb{C}^{n-1} \times \mathcal{A}_{n}
$$

be endowed with the product measure $\mu_{\Omega_{n}}$, normalized to have total unit mass (see Remark 2.32 below). Then, let

$$
\psi_{n}(\lambda, w, M, Q):=\left(\left(\begin{array}{cc}
\lambda & w^{*} \\
0 & M Q+\lambda I_{n-1}
\end{array}\right), \lambda, e_{1}\right) .
$$

Remark 2.32. An explicit description of the product measure $\mu_{\Omega_{n}}$ is as follows:

$$
\mu_{\Omega_{n}}(Y):=C_{n} \underset{\lambda, w}{\mathbb{E}} \mu_{\mathcal{A}_{n}}(\{(M, Q):(\lambda, w, M, Q) \in Y\})
$$

for measurable sets $Y \subseteq \Omega_{n}$, where $\lambda \sim \mathcal{N}_{\mathbb{C}}, w \sim \mathcal{N}_{\mathbb{C}^{n \times 1}}$ and $C_{n}$ is a normalizing constant given by

$$
C_{n}^{-1}=\underset{(\lambda, M, Q) \in \mathbb{C} \times \mathcal{A}_{n}}{\operatorname{Prob}}\left(2 \Re(\bar{\lambda} \operatorname{tr}(M Q)) \leq 1-|\lambda|^{2}(n-1)\right) .
$$

Our last main result (see Section 10 for a proof) is the following. 
Theorem 2.33. Let $\Omega_{n}$ and $\psi_{n}$ be as in Definition 2.31 for all $n \geq 2$. Then:

(1) Choosing $\omega \in \Omega_{n}$ can be done by choosing $2 n^{2}-2 n+1$ numbers with the $\mathcal{N}_{\mathbb{C}}$ distribution, checking a test which involves the computation of a Moore-Penrose inverse and computing two $Q R$ decompositions. This process may be repeated as a function of the test's outcome, but the expectation of the number of times the test is performed is at most $C_{n}$. The total expected running time is $\mathcal{O}\left(n^{3} C_{n}\right)$.

(2) Given $\omega \in \Omega_{n}$, computing $\psi_{n}(\omega)$ can be done with running time $\mathcal{O}\left(n^{3}\right)$.

(3) For any unitarily invariant measurable mapping $\phi: \mathcal{V} \rightarrow[0, \infty]$ we have

$$
\underset{\omega \sim \Omega_{n}}{\mathbb{E}} \phi\left(\psi_{n}(\omega)\right) \leq e n C_{n} \underset{A_{0} \sim \mathcal{N}_{\mathbb{C}^{n \times n}}}{\mathbb{E}} \frac{1}{n} \sum_{\lambda_{0}, v_{0}: A_{0} v_{0}=\lambda_{0} v_{0}} \phi\left(A_{0}, \lambda_{0}, v_{0}\right) .
$$

Note that (24) can be understood as follows: let $m_{1}$ be the push-forward measure of $\psi_{n}$ in $\mathcal{V}$ and let $m_{2}$ be the measure in $\mathcal{V}$ given by

$$
m_{2}(X)=\underset{A_{0} \sim \mathcal{N}_{\mathbb{C}^{n \times n}}}{\mathbb{E}} \frac{1}{n} \sharp\left\{\left(\lambda_{0}, v_{0}\right): A_{0} v_{0}=\lambda_{0} v_{0},\left(A_{0}, \lambda_{0}, v_{0}\right) \in X\right\}
$$

for any measurable set $X \subseteq \mathcal{V}$. Then the Radon-Nikodym derivative $d m_{1} / d m_{2}$ is bounded above by $e n C_{n}$.

Problem 2.34. Describe an alternative collection $\left(\Omega_{n}, \psi_{n}\right)$ which satisfies a sharper version of (24), with a constant in the place of $n C_{n}$. This would improve the running time of the algorithm Random_initial_triple below by a factor of $\mathcal{O}\left(n C_{n}\right)$.

We are now prepared to describe our random homotopy algorithm.

\begin{tabular}{l}
\hline Algorithm 5. Random_initial_triple \\
\hline Input: $A \in \mathbb{C}^{n \times n}$ \\
\hline Randomly choose $\omega \in \Omega_{n}$ \\
$\left(A_{0}, \lambda_{0}, v_{0}\right):=\psi_{n}(\omega) \quad\left(\right.$ note that $\left.v_{0}=e_{1}\right)$ \\
$(\zeta, w):=$ Path-follow $\left(A, A_{0}, \lambda_{0}, v_{0}\right)$ \\
\hline Output: $(\zeta, w) \in\left(\mathbb{C} \times \mathbb{C}^{n}\right)^{n}$ \\
Postconditions: The execution halts if the lifting of $\mathcal{L}_{A_{0}, A}$ at $\left(\lambda_{0}, v_{0}\right)$ does \\
not meet $\Sigma^{\prime}$. In this case, $(\zeta, w)$ is an approximate eigenpair of $A$.
\end{tabular}

From Theorem 2.33, the expected running time of the computation of $\left(A_{0}, \lambda_{0}, v_{0}\right)$ is $\mathcal{O}\left(n^{3} C_{n}\right)$. Moreover, the expected number of homotopy steps in the execution of Path-follow $\left(A, A_{0}, \lambda_{0}, v_{0}\right)$ is

$$
S=\underset{A \sim \mathcal{N}_{\mathbb{C}^{n \times n}}, \omega \sim \Omega_{n}}{\mathbb{E}} K\left(A, \psi_{n}(\omega)\right)=\underset{\omega \sim \Omega_{n}}{\mathbb{E}} \underset{A \sim \mathcal{N}_{\mathbb{C}^{n} \times n}}{\mathbb{E}} K\left(A, \psi_{n}(\omega)\right),
$$


where $K\left(A, \psi_{n}(\omega)\right)$ is as in Theorem 2.19. From (24), we have

$$
\begin{aligned}
S & \leq e n C_{n} \underset{A_{0} \sim \mathcal{N}_{\mathbb{C}^{n \times n}}}{\mathbb{E}} \frac{1}{n} \sum_{\lambda_{0}, v_{0}: A_{0} v_{0}=\lambda_{0} v_{0}} \underset{A \sim \mathcal{N}_{\mathbb{C}^{n \times n}}^{\mathbb{E}}}{\mathbb{E}} K\left(A, A_{0}, \lambda_{0}, v_{0}\right) \\
& \leq \mathcal{O}\left(n^{3} C_{n}\right) .
\end{aligned}
$$

We multiply the number of steps by $\mathcal{O}\left(n^{3}\right)$ to get the following complexity bound.

Theorem 2.35. The algorithm Random_initial_triple returns (almost surely) an approximate eigenpair of its input $A \in \mathbb{C}^{n \times n}$. Its average cost satisfies

$$
\operatorname{Avg} \operatorname{Cost}(n)=\mathcal{O}\left(n^{6} C_{n}\right) \underset{\text { Lem. } 10.1}{\leq} \mathcal{O}\left(n^{7}\right)
$$

\section{Some properties of the condition number $\mu$}

There is a general geometric framework for defining condition numbers $[19, \S 14.3]$. In our situation, this framework takes the following form.

If $(A, \lambda, v) \in \mathcal{W}$, then from the inverse function theorem the projection $\pi_{1}: \mathcal{V} \rightarrow \mathbb{C}^{n \times n}$ (cf. (3)), around $(A, \lambda, v)$, has a local inverse $\mathcal{U} \rightarrow \mathcal{V}, B \mapsto(B, G(B))$, that is defined on an open neighborhood $\mathcal{U}$ of $A$ in $\mathbb{C}^{n \times n}$. We call $G$ the solution map. The map $G$ decomposes as $G=\left(G_{\lambda}, G_{v}\right)$, where

$$
G_{\lambda}: \mathcal{U} \rightarrow \mathbb{C} \quad \text { and } \quad G_{v}: \mathcal{U} \rightarrow \mathbb{P}\left(\mathbb{C}^{n}\right)
$$

associate to matrices $B \in \mathcal{U}$ an eigenvalue and the corresponding eigenvector. Let

$$
D G_{\lambda}(A): \mathbb{C}^{n \times n} \rightarrow \mathbb{C} \text { and } D G_{v}(A): \mathbb{C}^{n \times n} \rightarrow v^{\perp}
$$

be the derivatives of these maps at $A$. The condition numbers for the eigenvalue $\lambda$ and the eigenvector $v$ of $A$ are defined as follows:

$$
\mu_{\lambda}(A, \lambda, v):=\left\|D G_{\lambda}(A)\right\| \quad \text { and } \quad \mu_{v}(A, \lambda, v):=\left\|D G_{v}(A)\right\|,
$$

where the norms are the operator norms with respect to the chosen norms (on $\mathbb{C}^{n \times n}$ we use the Frobenius norm and on $v^{\perp}$ the norm given by (1)).

Lemma 14.17 in [19] gives explicit descriptions of $D G_{\lambda}$ and $D G_{v}$. Before stating it, we recall that if $\lambda$ is an eigenvalue of $A$ there exists $u \in \mathbb{P}\left(\mathbb{C}^{n}\right)$ (the left eigenvector) such that $(A-\lambda \mathrm{Id})^{*} u=0$. Recall the linear map $A_{\lambda, v}: v^{\perp} \rightarrow v^{\perp}$ introduced in (4).

Lemma 3.1. Assume that $A v=\lambda v$ and $\lambda$ has multiplicity 1. Then the associated left eigenvector is

$$
u=v-i_{\mathbb{C}^{n}} A_{\lambda, v}^{-*} P_{v^{\perp}} A^{*} v .
$$

Here we have denoted $A_{\lambda, v}^{-*}:=\left(A_{\lambda, v}^{-1}\right)^{*}$. Note that $\langle u, v\rangle=\|v\|^{2}$.

Proof. Take a representative such that $\|v\|=1$ and let

$$
z:=i_{\mathbb{C}^{n}} A_{\lambda, v}^{-*} P_{v^{\perp}} A^{*} v \underset{(6)}{=}\left(\left(\mathrm{Id}-v v^{*}\right)(A-\lambda \mathrm{Id})^{*}\left(\mathrm{Id}-v v^{*}\right)\right)^{\dagger} A^{*} v .
$$


From the definition of the Moore-Penrose pseudoinverse, $z$ is the unique element in $v^{\perp}$ that minimizes $\left\|\left(\mathrm{Id}-v v^{*}\right)(A-\lambda \mathrm{Id})^{*} z-A^{*} v\right\|$, that is, we have $\left(\mathrm{Id}-v v^{*}\right)(A-\lambda \mathrm{Id})^{*} z=$ $P_{v^{\perp}}\left(A^{*} v\right)=\left(\mathrm{Id}-v v^{*}\right) A^{*} v$ or equivalently

$$
(A-\lambda \mathrm{ld})^{*} z=A^{*} v+t v \quad \text { for some } t \in \mathbb{C} .
$$

Premultiplying both sides by $v^{*}$ we have

$$
v^{*}(A-\lambda \mathrm{Id})^{*} z=v^{*} A^{*} v+t\|v\|^{2}, \quad \text { so } \quad 0=(\lambda v)^{*} v+t\|v\|^{2}=(\bar{\lambda}+t)\|v\|^{2},
$$

hence $t=-\bar{\lambda}$ and then

$$
(A-\lambda \mathrm{Id})^{*}(v-z)=(A-\lambda \mathrm{ld})^{*} v-A^{*} v+\bar{\lambda} v=0,
$$

that is, $v-z$ is a left eigenvector of $A$ with associated (left) eigenvalue $\bar{\lambda}$ as wanted.

The following is Lemma 14.17 in [19].

Lemma 3.2. Let $(A, \lambda, v) \in \mathcal{W}$ and let $u$ be a left eigenvector of $A$ with eigenvalue $\bar{\lambda}$. Then:

(a) $\langle v, u\rangle \neq 0$.

(b) The derivative of the solution map is given by $D G(A)(\dot{A})=(\dot{\lambda}, \dot{v})$, where

$$
\dot{\lambda}=\frac{\langle\dot{A} v, u\rangle}{\langle v, u\rangle}, \quad \dot{v}=-A_{\lambda, v}^{-1} P_{v^{\perp}} \dot{A} v .
$$

The following result, which follows directly from Lemma 3.2, was already pointed out in [56] (see also [19, Prop. 14.15]).

Proposition 3.3. Choosing the Frobenius norm on $T_{A} \mathbb{C}^{n \times n}=\mathbb{C}^{n \times n}$ and $\frac{1}{\|v\|}\|\cdot\|$ on $v^{\perp}$, the condition numbers $\mu_{v}$ for the eigenvector problem and $\mu_{\lambda}$ for the eigenvalue problem satisfy

$$
\mu_{\lambda}(A, \lambda, v)=\left\|D G_{\lambda}(A)\right\|=\frac{\|u\|\|v\|}{|\langle u, v\rangle|}=\frac{\|u\|}{(25)} \frac{\| \sqrt{1+\mu(A, \lambda, v)^{2}}}{\|v\|}
$$

and

$$
\mu_{v}(A, \lambda, v)=\left\|D G_{v}(A)\right\|=\left\|A_{\lambda, v}^{-1}\right\|=\frac{\mu(A, \lambda, v)}{\|A\|_{F}} .
$$

Proof of Proposition 2.7. The first two inequalities are immediate from Proposition 3.3. For the third one, note that

$$
\|\dot{\Gamma}(t)\|=\|(\dot{A}, \dot{\lambda}, \dot{v})\| \leq\|\dot{A}\| \sqrt{1+\mu_{t}^{2}+\left(1+\mu_{t}^{2}\right)} \leq\|\dot{A}\| \sqrt{6 \mu_{t}^{2}}
$$

the last inequality since $\mu_{t} \geq 1 / \sqrt{2}$ (Lemma 2.6).

Our last lemma is a version of Lemma 2.6 without the assumption that our point lies on $\mathcal{W}$.

Lemma 3.4. For $A \in \mathbb{C}^{n \times n}, w \in \mathbb{C}^{n}$ and $\zeta \in \mathbb{C}$ with $|\zeta| \leq\|A\|_{F}$ we have

$$
\mu(A, \zeta, w) \geq \frac{1}{1+\sqrt{1-\frac{\left\|w^{*} A\right\|^{2}}{\|w\|^{2}\|A\|_{F}^{2}}}} \geq \frac{1}{2} .
$$


Proof. We can assume that $w=e_{1}$ and write

$$
A=\left(\begin{array}{cc}
\lambda & a^{*} \\
b & \hat{A}
\end{array}\right),
$$

where $\lambda \in \mathbb{C}$ and $a, b \in \mathbb{C}^{n-1}$. Then $A_{\zeta, w} \equiv \hat{A}-\zeta \mathrm{Id}_{n-1}$ and

$$
\begin{aligned}
\frac{\mu\left(A, \zeta, e_{1}\right)}{\|A\|_{F}} & =\left\|\left(\hat{A}-\zeta \operatorname{ld}_{n-1}\right)^{-1}\right\| \geq \frac{1}{\left\|\hat{A}-\zeta \operatorname{ld}_{n-1}\right\|} \geq \frac{1}{\|\hat{A}\|+|\zeta|} \\
& \geq \frac{1}{\sqrt{\|A\|_{F}^{2}-\left\|e_{1}^{*} A\right\|^{2}}+\|A\|_{F}}
\end{aligned}
$$

The lemma follows.

\section{Proofs of Theorem 2.8 and Corollary 2.9}

It will be handy to use the definition of $\mu$ given in (10). We start with a very simple linear algebra lemma about the Moore-Penrose pseudoinverse.

Lemma 4.1. Let $R, R^{\prime} \in \mathbb{C}^{n \times n}$ be such that $R$ has rank $n-1$. Assume moreover that $\operatorname{det}\left(R^{\prime}\right)=0$ and

$$
\left\|R-R^{\prime}\right\| \leq \varepsilon /\left\|R^{\dagger}\right\| \quad \text { for some } 0 \leq \varepsilon<1 .
$$

Then $R^{\prime}$ has rank $n-1$ and

$$
\frac{\left\|R^{\dagger}\right\|}{1+\varepsilon} \leq\left\|R^{\prime \dagger}\right\| \leq \frac{\left\|R^{\dagger}\right\|}{1-\varepsilon}
$$

Proof. Let $\sigma$ and $\sigma^{\prime}$ be the smallest nonzero singular values of $R$ and $R^{\prime}$, respectively. Note that $\sigma-\left\|R-R^{\prime}\right\| \leq \sigma^{\prime} \leq \sigma+\left\|R-R^{\prime}\right\|$ (this is a classical fact proved for the first time in [58]; see also [29, Cor. 8.6.2]). In particular, $\sigma^{\prime} \geq \sigma-\left\|R-R^{\prime}\right\|=$ $\left\|R^{\dagger}\right\|^{-1}-\left\|R-R^{\prime}\right\|>0$, so $R^{\prime}$ has rank at least $n-1$ and by hypothesis it has rank $n-1$. Moreover, we have

$$
\left\|R^{\dagger}\right\|=\frac{1}{\sigma}=\frac{1}{\sigma^{\prime}} \frac{\sigma^{\prime}}{\sigma} \leq\left\|R^{\prime \dagger}\right\| \frac{\sigma+\left\|R-R^{\prime}\right\|}{\sigma} \leq(1+\varepsilon)\left\|R^{\prime \dagger}\right\| .
$$

The upper bound follows from a similar argument.

Proof of Theorem 2.8. Choose representatives such that $\|v\|=\left\|v^{\prime}\right\|=1$ and let

$$
Q:=\left(\mathrm{Id}-v v^{*}\right)(A-\lambda \mathrm{Id})\left(\mathrm{Id}-v v^{*}\right), \quad Q^{\prime}:=\left(\mathrm{Id}-v^{\prime} v^{\prime *}\right)\left(A^{\prime}-\lambda^{\prime} \mathrm{Id}\right)\left(\mathrm{Id}-v^{\prime} v^{*}\right) .
$$

We have $\operatorname{rank}(Q)=n-1$ since $\mu(A, \lambda, v)<\infty$ by our assumption (see (10)). We claim that

$$
\left\|Q-Q^{\prime}\right\| \leq \varepsilon /\left\|Q^{\dagger}\right\|
$$

which from Lemma 4.1 implies that $Q^{\prime}$ has rank $n-1$ and

$$
\frac{\left\|Q^{\dagger}\right\|}{1+\varepsilon} \leq\left\|Q^{\prime \dagger}\right\| \leq \frac{\left\|Q^{\dagger}\right\|}{1-\varepsilon}
$$


that is (recall $\|A\|_{F}=\left\|A^{\prime}\right\|_{F}=1$ ),

$$
\frac{\mu(A, \lambda, v)}{1+\varepsilon} \leq \mu\left(A^{\prime}, \lambda^{\prime}, v^{\prime}\right) \leq \frac{\mu(A, \lambda, v)}{1-\varepsilon},
$$

as wanted.

It remains to prove the claim. To do so, let $\left(A_{t}, \lambda_{t}, v_{t}\right)$ be a geodesic in $\mathbb{C}^{n \times n} \times \mathbb{C} \times$ $\mathbb{P}\left(\mathbb{C}^{n}\right)$, parametrized by arc-length, joining $(A, \lambda, v)$ and $\left(A^{\prime}, \lambda^{\prime}, v^{\prime}\right)$, so by hypothesis we have $t \in\left[0, \varepsilon /\left(4 \sqrt{3}\left\|Q^{\dagger}\right\|\right)\right]$ and we can choose representatives $v_{t}$ in such a way that

$$
\dot{v}_{t} \perp v_{t}, \quad\left\|v_{t}\right\|=1, \quad\left\|\dot{A}_{t}\right\|_{F}^{2}+\left|\dot{\lambda}_{t}\right|^{2}+\left\|\dot{v}_{t}\right\|^{2}=1, \quad \text { for all } t \in\left[0, \varepsilon /\left(4 \sqrt{3}\left\|Q^{\dagger}\right\|\right)\right] .
$$

Let $Q_{t}:=\left(\mathrm{Id}-v_{t} v_{t}^{*}\right)\left(A_{t}-\lambda_{t} \mathrm{Id}\right)\left(\mathrm{Id}-v_{t} v_{t}^{*}\right)$. In order to bound $\left\|\dot{Q}_{t}\right\|$ we first note that for $x \in \mathbb{C}^{n}$,

$$
\begin{aligned}
\left\|\left(\dot{v}_{t} v_{t}^{*}+v_{t} \dot{v}_{t}^{*}\right)(x)\right\| & =\left\|\dot{v}_{t}\left\langle x, v_{t}\right\rangle+v_{t}\left\langle x, \dot{v}_{t}\right\rangle\right\|=\sqrt{\left\|\dot{v}_{t}\right\|^{2}\left|\left\langle x, v_{t}\right\rangle\right|^{2}+\left\|v_{t}\right\|^{2}\left|\left\langle x, \dot{v}_{t}\right\rangle\right|^{2}} \\
& =\left\|\dot{v}_{t}\right\| \sqrt{\left|\left\langle x, v_{t}\right\rangle\right|^{2}+\left|\left\langle x, \dot{v}_{t} /\left\|\dot{v}_{t}\right\|\right\rangle\right|^{2}} \leq\left\|\dot{v}_{t}\right\|\|x\|,
\end{aligned}
$$

that is, $\left\|\dot{v}_{t} v_{t}^{*}+v_{t} \dot{v}_{t}^{*}\right\| \leq\left\|\dot{v}_{t}\right\|$. On the other hand,

$$
\left\|A_{t}-\lambda_{t} \mathrm{Id}\right\| \leq\left\|A_{t}\right\|+\left|\lambda_{t}\right| \leq\left\|A_{t}\right\|+\left\|A_{t}\right\| \leq 2\left\|A_{t}\right\|_{F}=2,
$$

so computing the derivative of $Q_{t}$ we see that

$$
\begin{aligned}
\left\|\dot{Q}_{t}\right\| & \leq 2\left\|\dot{v}_{t} v_{t}^{*}+v_{t} \dot{v}_{t}^{*}\right\|\left\|A_{t}-\lambda_{t} \mathrm{Id}\right\|+\left\|\dot{A}_{t}-\dot{\lambda}_{t} \mathrm{Id}\right\| \\
& \leq 4\left\|\dot{v}_{t}\right\|+\left\|\dot{A}_{t}-\dot{\lambda}_{t} \mathrm{Id}\right\| \leq 4\left(\left\|\dot{v}_{t}\right\|+\left\|\dot{A}_{t}\right\|+\left|\dot{\lambda}_{t}\right|\right) \\
& \leq 4 \sqrt{3}\left(\left\|\dot{v}_{t}\right\|^{2}+\left\|\dot{A}_{t}\right\|^{2}+\left|\dot{\lambda}_{t}\right|^{2}\right)^{1 / 2}=4 \sqrt{3} .
\end{aligned}
$$

Thus, we have $\left\|Q-Q^{\prime}\right\| \leq \int_{0}^{\varepsilon /\left(4 \sqrt{3}\left\|Q^{\dagger}\right\|\right)} 4 \sqrt{3} d t \leq 4 \sqrt{3} \varepsilon /\left(4 \sqrt{3}\left\|Q^{\dagger}\right\|\right)$, finishing the proof of (26) and that of Theorem 2.8.

Proof of Corollary 2.9. Consider the portion $\mathcal{L}_{A, A^{\prime}}=\left\{A_{t}\right\}$ of the great circle defined for $t \in[0, \alpha]$ where $\alpha=d_{\mathbb{S}}\left(A, A^{\prime}\right)$. Let $(\lambda, v)$ be any eigenpair of $A$. From the inverse function theorem, the map $t \mapsto \Gamma(t)=\left(A_{t}, \lambda_{t}, v_{t}\right) \in \mathcal{W}$ (given by the local inverse of $\left.\pi_{1}\right)$ is defined and, with $\operatorname{dist}(t):=\operatorname{dist}\left((A, \lambda, v),\left(A_{t}, \lambda_{t}, v_{t}\right)\right)$, satisfies

$$
\operatorname{dist}(t)<\frac{\varepsilon}{4 \sqrt{3} \mu(A, \lambda, v)}, \quad t \in[0, \delta),
$$

for some maximal $\delta \in(0, \alpha]$. From Theorem 2.8 this implies

$$
\mu(\Gamma(t)) \leq \frac{\mu(A, \lambda, v)}{1-\varepsilon}, \quad t \in[0, \delta),
$$

giving a global bound for the norm of the derivative of the solution map. Thus, $\Gamma(t)$ can be continuously extended to $[0, \delta]$ and from maximality of $\delta$, either $\delta=\alpha$ or $\delta<\alpha$ 
and (27) must become an equality when changing $t$ to $\delta$. In this last case, which we will rule out by contradiction, note that from Proposition 2.7 and (28),

$$
\operatorname{dist}(\delta) \leq \int_{0}^{\delta}\|\dot{\Gamma}(t)\| d t \leq \sqrt{6} \int_{0}^{\delta} \mu(\Gamma(t)) d t \leq \sqrt{6} \delta \frac{\mu(A, \lambda, v)}{1-\varepsilon} .
$$

Now, in our range of values, we have $\alpha=d_{\mathbb{S}}\left(A, A^{\prime}\right)=2 \arcsin \left(\left\|A-A^{\prime}\right\|_{F} / 2\right)<$ $\frac{1001}{1000}\left\|A-A^{\prime}\right\|_{F}$, which, together with $\delta<\alpha$, implies

$\operatorname{dist}(\delta)<\frac{1001\left\|A-A^{\prime}\right\|_{F} \sqrt{6} \mu(A, \lambda, v)}{1000(1-\varepsilon)} \leq \frac{1001 \varepsilon \sqrt{6}}{50000(1-\varepsilon) \mu(A, \lambda, v)}<\frac{\varepsilon}{4 \sqrt{3} \mu(A, \lambda, v)}$,

which is a contradiction (note that we have used the bound $\left\|A-A^{\prime}\right\|_{F} \mu(A, \lambda, v)^{2} \leq \varepsilon / 50$ and the fact that $\varepsilon<1 / 2$ ). We thus conclude that $\delta=\alpha$ and the corollary follows from (27) and Theorem 2.8.

\section{Condition-length homotopy continuation}

The goal of this section is to fully describe the routine Choose_step (with which the algorithm Path-follow will be complete) and to prove Theorem 2.19.

To do so, it will be useful to denote by $\beta(A, \zeta, w)$ the length (in the tangent space) of the Newton step with matrix $A \in \mathbb{S}$ and input $(\zeta, w) \in \mathbb{C} \times \mathbb{P}\left(\mathbb{C}^{n}\right)$. That is, if we take a representative such that $\|w\|=1$,

$$
\beta(A, \zeta, w)^{2}:=\left\|\left(\left.D F_{A}(\zeta, w)\right|_{\mathbb{C} \times w^{\perp}}\right)^{-1} F_{A}(\zeta, w)\right\|^{2}=\left\|\left(\begin{array}{c}
\dot{\lambda} \\
\dot{v}
\end{array}\right)\right\|^{2}=|\dot{\lambda}|^{2}+\|\dot{v}\|^{2},
$$

where $\dot{\lambda}, \dot{v}$ are given in (11). When $\|\dot{v}\|$ is small, $\beta$ approximates the length of the Newton step also under the distance dist. Indeed,

$$
\begin{aligned}
\operatorname{dist}_{A}\left((\zeta, w), N_{A}(\zeta, w)\right)^{2} & =|\dot{\lambda}|^{2}+d_{\mathbb{P}}(w, w-\dot{v})^{2} \\
& =|\dot{\lambda}|^{2}+\left(\arccos \frac{|\langle w, w-\dot{v}\rangle|}{\|w\|\|w-\dot{v}\|}\right)^{2} \\
& =|\dot{\lambda}|^{2}+\left(\arccos \frac{1}{\sqrt{1+\|\dot{v}\|^{2}}}\right)^{2} .
\end{aligned}
$$

It is easy to check that

$$
\frac{9}{10} x^{2} \leq\left(\arccos \frac{1}{\sqrt{1+x^{2}}}\right)^{2} \leq x^{2} \quad \text { for } x \leq \frac{1}{3},
$$

so we have proved that whenever $\|\dot{v}\| \leq 1 / 3$,

$$
\frac{9}{10} \beta(A, \zeta, w) \leq \operatorname{dist}_{A}\left((\zeta, w), N_{A}(\zeta, w)\right) \leq \beta(A, \zeta, w) .
$$

(The upper bound inequality is valid regardless of the value of $\|\dot{v}\|$.) The knowledge of $\beta$ allows us to bound the distance from an approximate eigenpair to the associated exact eigenpair. 
Lemma 5.1. Assume that $(\zeta, w)$ is an approximate eigenpair of $A \in \mathbb{S}$ with associated eigenpair $(\lambda, v)$. Then $\operatorname{dist}_{A}((\zeta, w),(\lambda, v)) \leq 2 \beta(A, \zeta, w)$. Moreover, if $\beta(A, \zeta, w) \leq 1 / 3$ we also have

Proof. We have

$$
\frac{1}{2} \beta(A, \zeta, w) \leq \operatorname{dist}_{A}((\zeta, w),(\lambda, v)) \leq 2 \beta(A, \zeta, w) .
$$

$$
\begin{aligned}
\operatorname{dist}_{A}((\zeta, w),(\lambda, v)) & \leq \operatorname{dist}_{A}\left((\zeta, w), N_{A}(\zeta, w)\right)+\operatorname{dist}_{A}\left(N_{A}(\zeta, w),(\lambda, v)\right) \\
& \leq \beta(A, \zeta, w)+\frac{1}{2} \operatorname{dist}_{A}((\zeta, w),(\lambda, v)),
\end{aligned}
$$

the last from the definition of approximate eigenpair. The upper bound in the statement follows. By a similar argument,

$$
\begin{aligned}
\operatorname{dist}_{A}((\zeta, w),(\lambda, v)) & \geq \operatorname{dist}_{A}\left((\zeta, w), N_{A}(\zeta, w)\right)-\operatorname{dist}_{A}\left(N_{A}(\zeta, w),(\lambda, v)\right) \\
& \underset{(29)}{\geq} \frac{9}{10} \beta(A, \zeta, w)-\frac{1}{2} \operatorname{dist}_{A}((\zeta, w),(\lambda, v)),
\end{aligned}
$$

and the lower bound follows as well.

\subsection{The Lipschitz property of $\beta$}

To describe Choose_step we need a set of constants satisfying a few relations. Not all of them are used in the description of Choose_step. Some only occur in the proof of the correctness of Path-follow. We will consider constants $c_{1}, c_{1}^{\prime}, c_{u}, c_{u}^{\prime}, c_{*}, c_{4}, c_{5}, c_{6}, c_{7}, K$. These are any collection of positive numbers satisfying the following:

$$
\begin{aligned}
& \sqrt{3} c_{1}^{\prime} \leq c_{1}<\frac{1}{3}, \quad \sqrt{3} c_{u}^{\prime} \leq c_{u}-\frac{\frac{3}{2} c_{1}^{2}(\sqrt{3}-1)}{1-3 c_{1}} \\
& 4 \sqrt{3} c_{*}<1, \quad c_{4}=c_{*}+\left(1+4 \sqrt{3} c_{*}\right)\left(c_{1}+2 c_{u}\right), \quad 4 \sqrt{3} c_{4}<1, \\
& \frac{2\left(1+4 \sqrt{3} c_{*}\right)}{1-4 \sqrt{3} c_{4}} c_{u}<K c_{*}<\frac{1}{5}, \quad c_{5}=c_{u}^{\prime}\left(1-4 \sqrt{3} c_{*}\right)-2 \frac{2 c_{*}+\frac{3}{2} c_{1}^{2}\left(1+4 \sqrt{3} c_{*}\right)}{\left(1-3 c_{1}\right)}, \\
& c_{6}=\frac{c_{5}\left(1-3 c_{1}\right)-2\left(1+3 c_{1}\right) c_{*}}{2\left(1+3 c_{1}\right)\left(1+4 \sqrt{3} c_{4}\right)}, \quad c_{7}=\min \left(\frac{c_{1}^{\prime}\left(1-4 \sqrt{3} c_{*}\right)}{\left(1+4 \sqrt{3} c_{4}\right)}, c_{6}\right) .
\end{aligned}
$$

A collection of parameters satisfying these constraints is shown in Table 1.

Table 1. Our choice of constants for Path-follow

\begin{tabular}{ll|ll}
\hline$c_{1}^{\prime}$ & $10^{-3}$ & $c_{4}$ & $0.005306 \ldots$ \\
$c_{1}$ & $\sqrt{3} c_{1}^{\prime}$ & $c_{5}$ & $0.00099 \ldots$ \\
$c_{u}^{\prime}$ & $10^{-3}$ & $c_{6}$ & $0.00038 \ldots$ \\
$c_{u}$ & $\sqrt{3} c_{u}^{\prime}+\frac{3 c_{1}^{2}(\sqrt{3}-1)}{2(1-3 c 1)}$ & $K$ & 64 \\
$c_{*}$ & $10^{-4}$ & $c_{7}$ & $0.00038 \ldots$ \\
\hline
\end{tabular}

In addition to these constants, for the sake of clarity, we spell out a working hypothesis that we will repeatedly use. 
Hypothesis 5.2. We have $(A, \lambda, v) \in \mathcal{V},\|A\|_{F}=1$, and $(\zeta, w)$ is an approximate eigenpair of A satisfying $|\zeta| \leq 1$ and

$$
\mu(A, \lambda, v) \operatorname{dist}_{A}((\lambda, v),(\zeta, w))<c_{*} .
$$

Also, $\theta:[0, \pi) \rightarrow \mathbb{S}, \theta(s)=A_{s}$, is some arc-length parametrized half-great circle with $A_{0}=A$.

It is easy to see from Theorem 2.12 that under Hypothesis 5.2 we have $\beta(A, \zeta, w)<1 / 3$ and in particular we can apply Lemma 5.1.

The main goal of this section is to prove the following result. Recall that we have defined

$$
F_{A}(\lambda, v)=(A-\lambda \mathrm{ld}) v
$$

Lemma 5.3. Under Hypothesis 5.2, let $s \leq c_{1} / \mu\left(A_{0}, \zeta, w\right)$ where $0 \leq c_{1}<1 / 3$. Let

$$
\Phi=\left\|\left(\left.D F_{A_{0}}(\zeta, w)\right|_{\mathbb{C} \times w^{\perp}}\right)^{-1} \dot{A}_{0} w\right\|
$$

Then

$$
\beta^{-}(s) \leq \beta\left(A_{s}, \zeta, w\right) \leq \beta^{+}(s),
$$

where

$$
\begin{aligned}
& \beta^{-}(s)=\frac{s \Phi-\beta\left(A_{0}, \zeta, w\right)-\frac{3}{2} c_{1}^{2} / \mu\left(A_{0}, \zeta, w\right)}{1+3 c_{1}} \\
& \beta^{+}(s)=\frac{s \Phi+\beta\left(A_{0}, \zeta, w\right)+\frac{3}{2} c_{1}^{2} / \mu\left(A_{0}, \zeta, w\right)}{1-3 c_{1}} .
\end{aligned}
$$

For the proof we need some stepping stones.

Lemma 5.4. Let $A \in \mathbb{S}, \zeta \in \mathbb{C}$, and $|\zeta| \leq 1, w \in \mathbb{C}^{n},\|w\|=1$. Then

$$
\left\|\left(\left.D F_{A}(\zeta, w)\right|_{\mathbb{C} \times w^{\perp}}\right)^{-1}\right\| \leq 3 \mu(A, \zeta, w) .
$$

Proof. This result is similar to [3, Proposition 6.6], but our assumptions are weaker and our definition of condition number is slightly different (see the proof of Theorem 2.12). We can assume that $w=e_{1}$ for the proof and write

$$
A=\left(\begin{array}{cc}
\lambda & a^{*} \\
b & \hat{A}
\end{array}\right)
$$

where $\lambda \in \mathbb{C}$ and $a, b \in \mathbb{C}^{n-1}$. Then, for $\dot{\zeta} \in \mathbb{C}$ and $\dot{w} \in \mathbb{C}^{n-1}$,

$$
\begin{aligned}
D F_{A}\left(\zeta, e_{1}\right)\left(\dot{\zeta},\left(\begin{array}{c}
0 \\
\dot{w}
\end{array}\right)\right) & =(A-\zeta \mathrm{Id})\left(\begin{array}{l}
0 \\
\dot{w}
\end{array}\right)-\dot{\zeta} e_{1}=\left(\begin{array}{cc}
\lambda-\zeta & a^{*} \\
b & \hat{A}-\zeta \mathrm{Id}_{n-1}
\end{array}\right)\left(\begin{array}{l}
0 \\
\dot{w}
\end{array}\right)-\dot{\zeta} e_{1} \\
& =\left(\begin{array}{cc}
-1 & a^{*} \\
0 & \hat{A}-\zeta \mathrm{Id}_{n-1}
\end{array}\right)\left(\begin{array}{c}
\dot{\zeta} \\
\dot{w}
\end{array}\right) .
\end{aligned}
$$


We thus have

$$
\begin{aligned}
\|\left(\left.D F_{A}(\zeta, w)\right|_{\left.\mathbb{C} \times w^{\perp}\right)^{-1} \|}\right. & =\left\|\left(\begin{array}{cc}
-1 & a^{*} \\
0 & \hat{A}-\zeta \operatorname{ld}_{n-1}
\end{array}\right)^{-1}\right\|=\left\|\left(\begin{array}{cc}
-1 & a^{*}\left(\hat{A}-\zeta \operatorname{ld}_{n-1}\right)^{-1} \\
0 & \left(\hat{A}-\zeta \operatorname{ld}_{n-1}\right)^{-1}
\end{array}\right)\right\| \\
& \leq\left\|\left(\begin{array}{cc}
0 & 0 \\
0 & \left(\hat{A}-\zeta \operatorname{ld}_{n-1}\right)^{-1}
\end{array}\right)\right\|+\left\|\left(\begin{array}{cc}
-1 & a^{*}\left(\hat{A}-\zeta \operatorname{ld}_{n-1}\right)^{-1} \\
0 & 0
\end{array}\right)\right\| \\
& \leq\left\|A_{\zeta, w}^{-1}\right\|+\sqrt{1+\|a\|^{2}\left\|A_{\zeta, w}^{-1}\right\|^{2}} \\
& =\mu(A, \zeta, w)+\sqrt{1+\|a\|^{2} \mu(A, \zeta, w)^{2}}
\end{aligned}
$$

the last two claims in this chain by definition of $A_{\zeta, w}$ and the fact that $\|A\|_{F}=1$. Now, from Lemma 3.4 we know that $\mu(A, \zeta, w) \geq\left(1+\sqrt{1-\|a\|^{2}}\right)^{-1}$, which implies

$$
\begin{aligned}
& \frac{\mu(A, \zeta, w)+\sqrt{1+\|a\|^{2} \mu(A, \zeta, w)^{2}}}{\mu(A, \zeta, w)}=1+\sqrt{\|a\|^{2}+\frac{1}{\mu(A, \zeta, w)^{2}}} \\
& \leq 1+\sqrt{\|a\|^{2}+\left(1+\sqrt{1-\|a\|^{2}}\right)^{2}}=1+\sqrt{2+2 \sqrt{1-\|a\|^{2}}} \leq 3,
\end{aligned}
$$

the last inequality due to $0 \leq\|a\| \leq\|A\|_{F}=1$. We have proved that

$$
\left\|\left(\left.D F_{A}(\zeta, w)\right|_{\mathbb{C} \times w^{\perp}}\right)^{-1}\right\| \leq \mu(A, \zeta, w)+\sqrt{1+\|a\|^{2} \mu(A, \zeta, w)^{2}} \leq 3 \mu(A, \zeta, w),
$$

and the bound claimed in the lemma follows.

Lemma 5.5. Under Hypothesis 5.2, let $s>0$ satisfy $3 \mu\left(A_{0}, \zeta, w\right) s<1$. Then

$$
\begin{gathered}
\left\|\left(\left.D F_{A_{s}}(\zeta, w)\right|_{\mathbb{C} \times w^{\perp}}\right)^{-1}\left(\left.D F_{A_{0}}(\zeta, w)\right|_{\mathbb{C} \times w^{\perp}}\right)\right\| \leq \frac{1}{1-3 \mu\left(A_{0}, \zeta, w\right) s}, \\
\left\|\left(\left.D F_{A_{0}}(\zeta, w)\right|_{\mathbb{C} \times w^{\perp}}\right)^{-1}\left(\left.D F_{A_{s}}(\zeta, w)\right|_{\mathbb{C} \times w^{\perp}}\right)\right\| \leq 1+3 \mu\left(A_{0}, \zeta, w\right) s .
\end{gathered}
$$

Proof. Note that

$$
\left.D F_{A_{0}}(\zeta, w)\right|_{\mathbb{C} \times w^{\perp}}(\dot{\eta}, \dot{x})=\left.D F_{A_{s}}(\zeta, w)\right|_{\mathbb{C} \times w^{\perp}}(\dot{\eta}, \dot{x})+\left(A_{0}-A_{s}\right) \dot{x},
$$

hence

$$
\begin{aligned}
\left\|\mathrm{ld}-\left(\left.D F_{A_{0}}(\zeta, w)\right|_{\mathbb{C} \times w^{\perp}}\right)^{-1}\left(\left.D F_{A_{s}}(\zeta, w)\right|_{\mathbb{C} \times w^{\perp}}\right)\right\| \\
\quad \leq \|\left(\left.D F_{A_{0}}(\zeta, w)\right|_{\left.\mathbb{C} \times w^{\perp}\right)^{-1}\|\| A_{s}-A_{0} \| \underset{\text { Lem. } 5.4}{\leq} 3 \mu\left(A_{0}, \zeta, w\right) s}\right.
\end{aligned}
$$

where we have used $\left\|A_{s}-A_{0}\right\| \leq\left\|A_{s}-A_{0}\right\|_{F} \leq s$. The second claim in the statement is now obvious and the first one follows from the Banach lemma, $\left\|(\mathrm{Id}+\Delta)^{-1}\right\| \leq$ $(1-\|\Delta\|)^{-1}$, valid for $\|\Delta\|<1$. 
Lemma 5.6. Under Hypothesis 5.2, let $s>0$ satisfy $3 \mu\left(A_{0}, \zeta, w\right) s<1$ and let

$$
\Phi_{\mathrm{aux}}(s):=\left\|\left(\left.D F_{A_{0}}(\zeta, w)\right|_{\mathbb{C} \times w^{\perp}}\right)^{-1}\left(A_{0}-A_{s}\right) w\right\| .
$$

Then

$$
\frac{\Phi_{\mathrm{aux}}(s)-\beta\left(A_{0}, \zeta, w\right)}{1+3 \mu\left(A_{0}, \zeta, w\right) s} \leq \beta\left(A_{s}, \zeta, w\right) \leq \frac{\Phi_{\mathrm{aux}}(s)+\beta\left(A_{0}, \zeta, w\right)}{1-3 \mu\left(A_{0}, \zeta, w\right) s}
$$

Proof. From the definition,

$$
\begin{aligned}
\beta\left(A_{s}, \zeta, w\right)= & \left\|\left(\left.D F_{A_{s}}(\zeta, w)\right|_{\mathbb{C} \times w^{\perp}}\right)^{-1} F_{A_{s}}(\zeta, w)\right\| \\
= & \|\left[( D F _ { A _ { s } } ( \zeta , w ) | _ { \mathbb { C } \times w ^ { \perp } } ) ^ { - 1 } \left(\left.D F_{A_{0}}(\zeta, w)\right|_{\left.\left.\mathbb{C} \times w^{\perp}\right)\right]}\right.\right. \\
& \cdot\left(\left.D F_{A_{0}}(\zeta, w)\right|_{\mathbb{C} \times w^{\perp}}\right)^{-1}\left(F_{A_{0}}(\zeta, w)+\left(A_{s}-A_{0}\right) w\right) \|
\end{aligned}
$$

Thus,

$$
\beta\left(A_{s}, \zeta, w\right) \leq\left\|\left(\left.D F_{A_{s}}(\zeta, w)\right|_{\mathbb{C} \times w^{\perp}}\right)^{-1}\left(\left.D F_{A_{0}}(\zeta, w)\right|_{\mathbb{C} \times w^{\perp}}\right)\right\|\left(\Phi_{\mathrm{aux}}(s)+\beta\left(A_{0}, \zeta, w\right)\right) .
$$

Similarly, we have

$$
\beta\left(A_{s}, \zeta, w\right) \geq \frac{\Phi_{\mathrm{aux}}(s)-\beta\left(A_{0}, \zeta, w\right)}{\left\|\left(\left.D F_{A_{0}}(\zeta, w)\right|_{\mathbb{C} \times w^{\perp}}\right)^{-1}\left(\left.D F_{A_{s}}(\zeta, w)\right|_{\mathbb{C} \times w^{\perp}}\right)\right\| .}
$$

The statement now follows from Lemma 5.5.

Proof of Lemma 5.3. We claim that we can write

$$
A_{s}=A_{0}+s \dot{A}_{0}+\frac{1}{2} s^{2} B
$$

for some $B$ with $\|B\|_{F} \leq 1$. Indeed, this is an elementary observation which follows from the fact that $A_{s}$ is a great circle in the sphere (one can choose $A_{s}$ to be the circle parametrized by $(\cos s, \sin s, 0, \ldots, 0)$ to prove it). As a consequence we have

$$
\begin{aligned}
\left|\Phi_{\text {aux }}(s)-s \Phi\right| & \leq\left\|\left(\left.D F_{A_{0}}(\zeta, w)\right|_{\mathbb{C} \times w^{\perp}}\right)^{-1}\left(A_{0}-A_{s}+s \dot{A}_{0}\right) w\right\| \\
& \leq \frac{1}{2} s^{2}\left\|\left(\left.D F_{A_{0}}(\zeta, w)\right|_{\mathbb{C} \times w^{\perp}}\right)^{-1}\right\| \underset{\text { Lem. 5.4 }}{\leq} \frac{3}{2} s^{2} \mu\left(A_{0}, \zeta, w\right) .
\end{aligned}
$$

The upper and lower bounds for $\beta\left(A_{s}, \zeta, w\right)$ in Lemma 5.3 now follow from this last estimate and Lemma 5.6.

\subsection{The step's length in the homotopy continuation}

The following result is crucial for the understanding of the homotopy algorithm. Its proof follows a logic which is similar to that of the proof of Corollary 2.9. 
Proposition 5.7. Under Hypothesis 5.2, let $s^{\prime}>0$ be any number such that

$$
c_{1}^{\prime} \leq \mu(A, \zeta, w) s^{\prime} \leq c_{1}
$$

and let $s^{\prime \prime}$ be any number such that

$$
c_{u}^{\prime} \leq \mu(A, \zeta, w) \beta^{+}\left(s^{\prime \prime}\right) \leq c_{u},
$$

where $\beta^{+}$is as in Lemma 5.3. Let $\bar{s}=\min \left(s^{\prime}, s^{\prime \prime}\right)$. Then

I. The (continuous) branch of the solution map $\pi_{1}^{-1} \circ \theta:[0, \bar{s}] \rightarrow \mathcal{V}$ with $\pi_{1}^{-1} \circ \theta(0)=$ $(A, \lambda, v)$ is well defined.

II. For every $s \in[0, \bar{s}]$, let $\left(A_{s}, \lambda_{s}, v_{s}\right):=\pi_{1}^{-1} \circ \theta(s)$. Then

$$
\frac{1}{1+4 \sqrt{3} c_{4}} \mu(A, \lambda, v)<\mu\left(A_{s}, \lambda_{s}, v_{s}\right)<\frac{\mu(A, \lambda, v)}{1-4 \sqrt{3} c_{4}}
$$

and

$$
\operatorname{dist}_{A_{s}}\left(\left(\lambda_{s}, v_{s}\right),(\zeta, w)\right)<\frac{K c_{*}}{\mu\left(A_{s}, \lambda_{s}, v_{s}\right)} .
$$

In particular, $(\zeta, w)$ is an approximate eigenpair of $A_{s}$ with associated eigenpair $\left(\lambda_{s}, v_{s}\right)$ for every $s \in[0, \bar{s}]$. Finally, the condition length $L_{\mu, 0, \bar{s}}\left(\pi_{1}^{-1}\left(A_{s}\right)\right)($ see $(18))$ of the curve $\pi_{1}^{-1}\left(A_{s}\right)$ is at least $c_{7}$.

Before we prove Proposition 5.7 we make a few comments on how the proofs of the proposition and Theorem 2.19 proceed. The hypotheses $\beta^{+}\left(s^{\prime \prime}\right)$ give us a bound on the distance from $(\zeta, w)$ to $\left(\lambda_{s}, v_{s}\right)$ and ultimately $(\lambda, v)$ to $\left(\lambda_{s}, v_{s}\right)$. Together with the bound on $s^{\prime}$ this allows us to invoke Theorems 2.12 and 2.8 to prove conclussions I and II of the proposition. The tricky part will be to prove the last statement that $L_{\mu, 0, \bar{s}}\left(\pi_{1}^{-1}\left(A_{s}\right)\right) \geq c_{7}$. This statement gives us the upper bound on the number of steps in Theorem 2.19 as the condition length divided by $c_{7}$. Now to see that

$$
\int_{0}^{\bar{s}} \mu\left(A_{s}, \lambda_{s}, v_{s}\right)\left\|\left(\dot{A}_{s}, \dot{\lambda}_{s}, \dot{v}_{s}\right)\right\| d s=L_{\mu, 0, \bar{s}}\left(\pi_{1}^{-1}\left(A_{s}\right)\right) \geq c_{7}
$$

it will suffice to prove that

$$
\int_{0}^{\bar{s}}\left\|\left(\dot{A}_{s}, \dot{\lambda}_{s}, \dot{v}_{s}\right)\right\| d s \geq \frac{\text { const }}{\mu(A, \lambda, v)},
$$

since $\mu$ is almost constant on the interval. For this it will suffice to prove that one of

$$
\int_{0}^{\bar{s}}\left\|\dot{A}_{s}\right\| d s \quad \text { or } \quad \int_{0}^{\bar{s}}\left\|\dot{v}_{s}\right\| d s
$$

is greater than const $/ \mu(A, \lambda, v)$. The first integral is $\bar{s}$. We will see that if $\bar{s}$ is small then $\operatorname{dist}_{A}\left(v_{s}, v\right)$ is greater than or equal to some constant over $\mu(A, \lambda, v)$, and so is the integral of $\left\|\dot{v}_{s}\right\|$ which is the length of a path between $v$ and $v_{s}$.

Proof of Proposition 5.7. We prove the first part of the proposition. From the inverse function theorem and the continuity of $\mu$, there exists a maximal $s^{*} \leq \bar{s}$ such that I and II hold with $[0, \bar{s}]$ changed to $\left[0, s^{*}\right)$. The global upper bound for $\mu\left(\pi_{1}^{-1}\left(A_{s}\right)\right)$ shown in (31) 
is in turn an upper bound for the derivative of the solution map, for $s \in\left[0, s^{*}\right)$. A standard limit argument in compact sets then implies that $\pi_{1}^{-1} \circ \theta$ can be extended in a continuous manner to $\left[0, s^{*}\right]$, and because (31) and (32) are open conditions, we must have one of the two following scenarios:

(i) $s_{*}=\bar{s}$ and both (31) and (32) hold with $s_{*}$ in place of $s$, or

(ii) at least one of (31) and (32) does not hold with $s_{*}$ in place of $s$.

We now discard the second option. Note that from Hypothesis 5.2 and Theorem 2.8,

$$
\frac{1}{1+4 \sqrt{3} c_{*}} \mu(A, \lambda, v)<\mu(A, \zeta, w)<\frac{\mu(A, \lambda, v)}{1-4 \sqrt{3} c_{*}} .
$$

Then, for every $s \in\left[0, s^{*}\right]$,

$$
\operatorname{dist}\left((A, \zeta, w),\left(A_{s}, \zeta, w\right)\right)=\left\|A-A_{s}\right\|_{F}<s^{*} \leq s^{\prime} \leq \frac{c_{1}}{\mu(A, \zeta, w)} \leq \frac{\left(1+4 \sqrt{3} c_{*}\right) c_{1}}{\mu(A, \lambda, v)},
$$

and because $s^{*} \leq s^{\prime \prime}$, from Lemma 5.3 we have

$$
\beta\left(A_{s}, \zeta, w\right) \leq \beta^{+}(s) \leq \frac{c_{u}}{\mu(A, \zeta, w)}<\frac{\left(1+4 \sqrt{3} c_{*}\right) c_{u}}{\mu(A, \lambda, v)}
$$

From Lemma 5.1 (recall that from II and Theorem 2.12, $(\zeta, w)$ is an approximate eigenpair of $A_{s}$ with associated eigenpair $\left(\lambda_{s}, v_{s}\right)$ for $\left.s \leq s_{*}\right)$, this last inequality implies

$$
\operatorname{dist}_{A_{s}}\left((\zeta, w),\left(\lambda_{s}, v_{s}\right)\right) \leq 2 \beta\left(A_{s}, \zeta, w\right)<\frac{2\left(1+4 \sqrt{3} c_{*}\right) c_{u}}{\mu(A, \lambda, v)} .
$$

We have thus proved that for every $s \in\left[0, s^{*}\right]$,

$$
\begin{aligned}
\operatorname{dist}\left((A, \lambda, v),\left(A_{s}, \lambda_{s}, v_{s}\right)\right) \leq & \operatorname{dist}_{A}((\lambda, v),(\zeta, w))+\operatorname{dist}\left((A, \zeta, w),\left(A_{s}, \zeta, w\right)\right) \\
& +\operatorname{dist}_{A_{s}}\left((\zeta, w),\left(\lambda_{s}, v_{s}\right)\right) \\
& <\frac{c_{*}}{\mu(A, \lambda, v)}+\frac{\left(1+4 \sqrt{3} c_{*}\right) c_{1}}{\mu(A, \lambda, v)}+\frac{2\left(1+4 \sqrt{3} c_{*}\right) c_{u}}{\mu(A, \lambda, v)} \\
& =\frac{c_{4}}{\mu(A, \lambda, v)} .
\end{aligned}
$$

Then, from Theorem 2.8 (note the strict inequality in the displayed formula above),

$$
\frac{1}{1+4 \sqrt{3} c_{4}} \mu(A, \lambda, v)<\mu\left(A_{s^{*}}, \lambda_{s^{*}}, v_{s^{*}}\right)<\frac{\mu(A, \lambda, v)}{1-4 \sqrt{3} c_{4}} .
$$

Thus, (31) holds at $s^{*}$ and moreover

$$
\operatorname{dist}_{A_{s^{*}}}\left(\left(\lambda_{s^{*}}, v_{s^{*}}\right),(\zeta, w)\right) \underset{(34),(35)}{<} \frac{2\left(1+4 \sqrt{3} c_{*}\right) c_{u}}{\left(1+4 \sqrt{3} c_{4}\right) \mu\left(A_{s^{*}}, \lambda_{s^{*}}, v_{s^{*}}\right)}<\frac{K c_{*}}{\mu\left(A_{s^{*}}, \lambda_{s^{*}}, v_{s^{*}}\right)} .
$$

That is, (32) holds at $s^{*}$ and we can discard option (ii), proving the first part of the proposition. 
For the last claim of the proposition, note that the condition length of the curve $\pi_{1}^{-1}\left(A_{s}\right)$ is

$$
\begin{aligned}
L_{\mu, 0, \bar{s}}\left(\pi_{1}^{-1}\left(A_{s}\right)\right) & =\int_{0}^{\bar{s}} \mu\left(\pi_{1}^{-1}\left(A_{s}\right)\right)\left\|D \pi_{1}^{-1}\left(A_{s}\right) \dot{A}_{s}\right\| d s \\
& \underset{(31)}{\geq} \frac{\mu(A, \lambda, v)}{1+4 \sqrt{3} c_{4}} \int_{0}^{\bar{s}}\left\|D \pi_{1}^{-1}\left(A_{s}\right) \dot{A}_{s}\right\| d s .
\end{aligned}
$$

If $\bar{s}=s^{\prime}$ then using the fact that the integrand is greater than or equal to 1 , and (33), we have

$$
L_{\mu, 0, \bar{s}}\left(\pi_{1}^{-1}\left(A_{s}\right)\right) \geq \frac{\mu(A, \lambda, v)}{1+4 \sqrt{3} c_{4}} s^{\prime} \geq \frac{c_{1}^{\prime}}{1+4 \sqrt{3} c_{4}} \frac{\mu(A, \lambda, v)}{\mu(A, \zeta, w)} \geq \frac{c_{1}^{\prime}\left(1-4 \sqrt{3} c_{*}\right)}{\left(1+4 \sqrt{3} c_{4}\right)} .
$$

Assume now that $\bar{s}=s^{\prime \prime}$. Then

$$
\begin{aligned}
L_{\mu, 0, \bar{s}}\left(\pi_{1}^{-1}\left(A_{s}\right)\right) & \geq \frac{\mu(A, \lambda, v)}{1+4 \sqrt{3} c_{4}} \int_{0}^{\bar{s}}\left\|D \pi_{1}^{-1}\left(A_{s}\right) \dot{A}_{s}\right\| d s \\
& \geq \frac{\mu(A, \lambda, v)}{1+4 \sqrt{3} c_{4}} \operatorname{dist}\left(\left(A_{0}, \lambda_{0}, v_{0}\right),\left(A_{\bar{s}}, \lambda_{\bar{s}}, v_{\bar{s}}\right)\right) .
\end{aligned}
$$

Now, note that $\left(\right.$ recall $\left.\left(A_{0}, \lambda_{0}, v_{0}\right)=(A, \lambda, v)\right)$

$$
\begin{aligned}
\operatorname{dist}\left(\left(A_{0}, \lambda_{0}, v_{0}\right)\right. & \left.,\left(A_{\bar{s}}, \lambda_{\bar{s}}, v_{\bar{S}}\right)\right) \\
& \geq \operatorname{dist}\left(\left(A_{0}, \zeta, w\right),\left(A_{\bar{s}}, \lambda_{\bar{s}}, v_{\bar{s}}\right)\right)-\operatorname{dist}_{A_{0}}\left(\left(\lambda_{0}, v_{0}\right),(\zeta, w)\right) \\
& \geq \operatorname{dist}_{A_{\bar{s}}}\left((\zeta, w),\left(\lambda_{\bar{s}}, v_{\bar{s}}\right)\right)-c_{*} / \mu(A, \lambda, v) .
\end{aligned}
$$

We need a lower bound for this last term. We first note that from Lemma 5.1 and Hypothesis 5.2,

$$
\beta(A, \zeta, w) \leq \frac{2 c_{*}}{\mu(A, \lambda, v)} \leq \frac{2 c_{*}}{\left(1-4 \sqrt{3} c_{*}\right) \mu(A, \zeta, w)},
$$

the last by (33). Using this last bound and, again, Lemmas 5.1 and 5.3, we have

$$
\begin{aligned}
& 2 \frac{1+3 c_{1}}{1-3 c_{1}} \operatorname{dist}_{A_{\bar{s}}}\left((\zeta, w),\left(\lambda_{\bar{s}}, v_{\bar{s}}\right)\right) \geq \frac{1+3 c_{1}}{1-3 c_{1}} \beta\left(A_{\bar{s}}, \zeta, w\right) \\
& \quad \geq \frac{1+3 c_{1}}{1-3 c_{1}} \beta^{-}(\bar{s})=\beta^{+}(\bar{s})-2 \frac{\beta(A, \zeta, w)+\frac{3}{2} c_{1}^{2} / \mu(A, \zeta, w)}{1-3 c_{1}} \\
& \\
& \geq \frac{c_{u}^{\prime}}{\mu(A, \zeta, w)}-2 \frac{\beta(A, \zeta, w)+\frac{3}{2} c_{1}^{2} / \mu(A, \zeta, w)}{1-3 c_{1}} \\
& \geq \frac{c_{u}^{\prime}\left(1-4 \sqrt{3} c_{*}\right)}{\mu(A, \lambda, v)}-2 \frac{2 c_{*}+\frac{3}{2} c_{1}^{2}\left(1+4 \sqrt{3} c_{*}\right)}{\left(1-3 c_{1}\right) \mu(A, \lambda, v)}=\frac{c_{5}}{\mu(A, \lambda, v)}
\end{aligned}
$$

For the inequality in the third line we have used the assumption in the statement and $s=s^{\prime \prime}$. For the inequality in the fourth line, we have used (33) and (36). For the equality in the fourth line we have used the definition of $c_{5}$. 
We have thus shown that if $\bar{s}=s^{\prime \prime}$, then

$$
\begin{aligned}
L_{\mu, 0, \bar{s}}\left(\pi_{1}^{-1}\left(A_{s}\right)\right) & \geq \frac{\mu(A, \lambda, v)}{1+4 \sqrt{3} c_{4}}\left(\frac{c_{5}\left(1-3 c_{1}\right)}{2\left(1+3 c_{1}\right) \mu(A, \lambda, v)}-\frac{c_{*}}{\mu(A, \lambda, v)}\right) \\
& =\frac{c_{5}\left(1-3 c_{1}\right)-2\left(1+3 c_{1}\right) c_{*}}{2\left(1+3 c_{1}\right)\left(1+4 \sqrt{3} c_{4}\right)}=c_{6} .
\end{aligned}
$$

Since $c_{7} \leq c_{6}$, the proof is complete.

We can finally describe the subroutine Choose_step. It is important to note that given any matrix $A \in \mathbb{C}^{n \times n}$, one can compute in $\mathcal{O}\left(n^{3}\right)$ arithmetic operations, for example by first reducing $A$ to tridiagonal Hessenberg form and then using the main result of [31], a number $r$ such that $\|A\| \leq r \leq \sqrt{3}\|A\|$. That is, we can compute operator norms within a factor of $\sqrt{3}$, and consequently we can compute $\mu$ within a factor of $\sqrt{3}$.

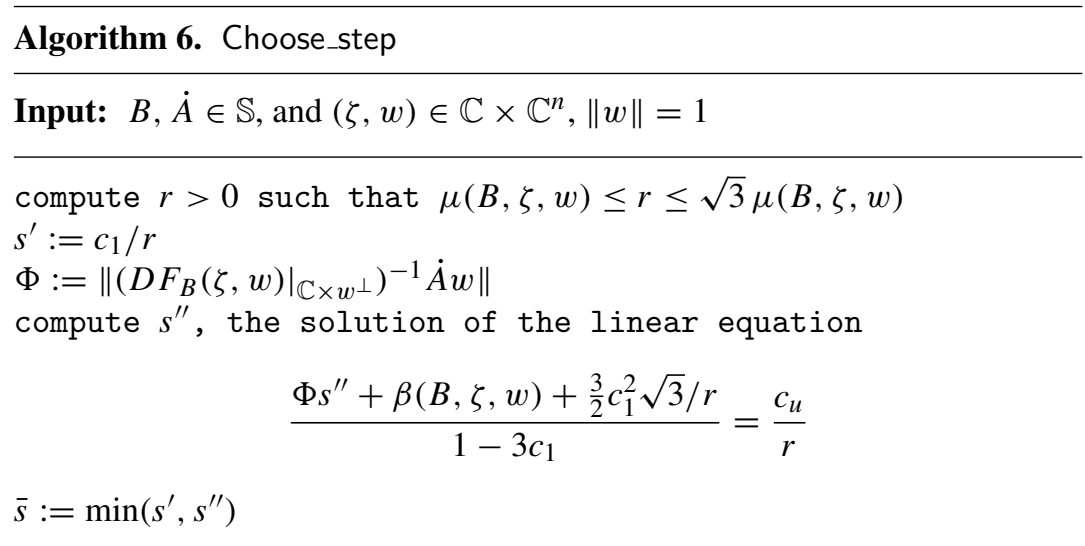

Output: $\Delta s=\bar{s} \in[0, \pi]$

The step size computed by Choose_step cannot be too small, as we show now.

Proposition 5.8. The value $\Delta s$ returned by Choose_step $(B, \dot{A}, \zeta, w)$ satisfies

$$
\Delta s \geq R / \mu(B, \zeta, w)^{2} \quad \text { with } \quad R=c_{7}\left(1-4 \sqrt{3} c_{4}\right)^{2} / 6>0 .
$$

\subsection{Proof of Theorem 2.19 and Proposition 5.8}

We now prove Theorem 2.19, and Proposition 5.8 will follow straightforwardly from our arguments.

From the definition of Path-follow it is clear that we can assume that $\left\|A_{0}\right\|_{F}=$ $\|A\|_{F}=1$. We further assume that the constants $c_{1}^{\prime}, c_{1}, c_{u}^{\prime}, c_{u} c_{*}, c_{4}, c_{5}, c_{6}, c_{7}$ and $K$ take the values in Table 1 and denote by $\pi_{1}^{-1}\left(\mathcal{L}_{A_{0}, A}\right)$ the lift of $\mathcal{L}_{A_{0}, A}$ with origin $\left(A_{0}, \lambda_{0}, v_{0}\right)$. Note that this lift is well defined since, by hypothesis, $\mathcal{L}_{A_{0}, A} \cap \Sigma=\emptyset$.

Let $B_{i}$ be the matrix $B$ at the beginning of the $i$ th iteration of Path-follow. Also, let $\left(\lambda_{i}, v_{i}\right)$ be such that $\left(B_{i}, \lambda_{i}, v_{i}\right)$ is the (unique) triple in $\pi_{1}^{-1}\left(\mathcal{L}_{A_{0}, A}\right)$ above $B_{i}$. 
We first prove that for all $i \geq 0,\left(\zeta_{i}, w_{i}\right)$ is an approximate zero of $B_{i}$ with associated eigenpair $\left(\lambda_{i}, v_{i}\right)$ and satisfies

$$
\operatorname{dist}_{B_{i}}\left(\left(\zeta_{i}, w_{i}\right),\left(\lambda_{i}, v_{i}\right)\right)<c_{*} / \mu\left(B_{i}, \lambda_{i}, v_{i}\right) .
$$

We reason by induction. The step $i=0$ is true by hypothesis (recall Definition 2.17). For the induction step, note that the $s^{\prime}$ defined by Choose_step satisfies (we omit the subscripts $i$ in $\left(B_{i}, \zeta_{i}, w_{i}\right)$ in the next few lines)

$$
\frac{c_{1}^{\prime}}{\mu(B, \zeta, w)} \leq \frac{c_{1}}{\sqrt{3} \mu(B, \zeta, w)} \leq s^{\prime} \leq \frac{c_{1}}{\mu(B, \zeta, w)} .
$$

Moreover, $s^{\prime \prime}$ satisfies

$$
\begin{aligned}
\beta^{+}\left(s^{\prime \prime}\right) & =\frac{\Phi s^{\prime \prime}+\beta(B, \zeta, w)+\frac{3}{2} c_{1}^{2} / \mu(B, \zeta, w)}{1-3 c_{1}} \\
& \leq \frac{\Phi s^{\prime \prime}+\beta(B, \zeta, w)+\frac{3}{2} c_{1}^{2} \sqrt{3} / r}{1-3 c_{1}}=\frac{c_{u}}{r} \leq \frac{c_{u}}{\mu(B, \zeta, w)}
\end{aligned}
$$

and

$$
\begin{aligned}
\beta^{+}\left(s^{\prime \prime}\right) & =\frac{\Phi s^{\prime \prime}+\beta(B, \zeta, w)+\frac{3}{2} c_{1}^{2} / \mu(B, \zeta, w)}{1-3 c_{1}} \geq \frac{\Phi s^{\prime \prime}+\beta(B, \zeta, w)+\frac{3}{2} c_{1}^{2} / r}{1-3 c_{1}} \\
& =\frac{c_{u}-\frac{3}{2} c_{1}^{2}(\sqrt{3}-1) /\left(1-3 c_{1}\right)}{r} \geq \frac{c_{u}-\frac{3}{2} c_{1}^{2}(\sqrt{3}-1) /\left(1-3 c_{1}\right)}{\sqrt{3} \mu(B, \zeta, w)} \geq \frac{c_{u}^{\prime}}{\mu(B, \zeta, w)} .
\end{aligned}
$$

We are thus under the hypothesis of Proposition 5.7 with $\bar{s}=\Delta s$ (by construction in Choose_step), which guarantees that $\left(\zeta_{i}, w_{i}\right)$ is an approximate eigenpair of the matrix $B_{i+1}$. Moreover, Proposition 5.7 also implies

$$
\operatorname{dist}_{B_{i+1}}\left(\left(\zeta_{i}, w_{i}\right),\left(\lambda_{i+1}, v_{i+1}\right)\right)<K c_{*} / \mu\left(B_{i+1}, \lambda_{i+1}, v_{i+1}\right) .
$$

Since $K c_{*}<1 / 5$, we deduce (using Theorem 2.12) that $\left(\zeta_{i}, w_{i}\right)$ is an approximate eigenpair of $B_{i+1}$ with associated eigenpair $\left(\lambda_{i+1}, v_{i+1}\right)$. Consequently, after three steps of Newton iteration, $\left(\zeta_{i+1}, w_{i+1}\right)$ satisfies (recall $K=64$ )

$$
\begin{aligned}
\operatorname{dist}_{B_{i+1}}\left(\left(\zeta_{i+1}, w_{i+1}\right),\left(\lambda_{i+1}, v_{i+1}\right)\right) & <\frac{1}{2^{2^{3}-1}} \frac{64 c_{*}}{\mu\left(B_{i+1}, \lambda_{i+1}, v_{i+1}\right)} \\
& =\frac{c_{*}}{2 \mu\left(B_{i+1}, \lambda_{i+1}, v_{i+1}\right)} .
\end{aligned}
$$

If $\left|\zeta_{i+1}\right| \leq 1$, this finishes the induction step. Otherwise, the algorithm divides $\zeta_{i+1}$ by its norm; in that case we have

$$
\begin{aligned}
\operatorname{dist}_{B_{i+1}}\left(\left(\frac{\zeta_{i+1}}{\left|\zeta_{i+1}\right|}, w_{i+1}\right),\left(\lambda_{i+1}, v_{i+1}\right)\right) \leq & \operatorname{dist}_{B_{i+1}}\left(\left(\frac{\zeta_{i+1}}{\left|\zeta_{i+1}\right|}, w_{i+1}\right),\left(\zeta_{i+1}, w_{i+1}\right)\right) \\
& +\operatorname{dist}_{B_{i+1}}\left(\left(\zeta_{i+1}, w_{i+1}\right),\left(\lambda_{i+1}, v_{i+1}\right)\right) \\
\leq & \left|\zeta_{i+1}\right|-1+\frac{c_{*}}{2 \mu\left(B_{i+1}, \lambda_{i+1}, v_{i+1}\right)}
\end{aligned}
$$


On the other hand, from (37) we have (use $\left|\lambda_{i+1}\right| \leq\left\|B_{i+1}\right\|_{F}=1$ )

$$
\left|\zeta_{i+1}-\lambda_{i+1}\right|<\frac{c_{*}}{2 \mu\left(B_{i+1}, \lambda_{i+1}, v_{i+1}\right)}, \quad \text { so } \quad\left|\zeta_{i+1}\right|<1+\frac{c_{*}}{2 \mu\left(B_{i+1}, \lambda_{i+1}, v_{i+1}\right)},
$$

hence

$$
\operatorname{dist}_{B_{i+1}}\left(\left(\frac{\zeta_{i+1}}{\left|\zeta_{i+1}\right|}, w_{i+1}\right),\left(\lambda_{i+1}, v_{i+1}\right)\right)<\frac{c_{*}}{\mu\left(B_{i+1}, \lambda_{i+1}, v_{i+1}\right)},
$$

and the induction step is finished also in the case $\left|\zeta_{i+1}\right|>1$.

The induction step is complete. In particular, this shows the last part of the statement.

To show the complexity bounds, assume Path-follow has performed $q+\ell$ iterations and let $0=s_{0}<s_{1}<\cdots<s_{q}<\cdots<s_{q+\ell}$ be the corresponding values of $s$. Then

$$
\begin{aligned}
L_{\mu, s_{q}, s_{q+\ell}}\left(\pi_{1}^{-1}\left(B_{s}\right)\right) & =\sum_{i=1}^{\ell} \int_{s_{q+i-1}}^{s_{q+i}} \mu\left(A_{s}, \lambda_{s}, v_{s}\right)\left\|\left(\dot{A}_{s}, \dot{\lambda}_{s}, \dot{v}_{s}\right)\right\| d s \\
& =\sum_{i=1}^{\ell} L_{\mu, s_{q+i-1}, s_{q+i}}\left(\pi_{1}^{-1}\left(A_{s}\right)\right) \geq \ell c_{7},
\end{aligned}
$$

the inequality by the last claim of Proposition 5.7. But the algorithm halts as soon as $s_{q+\ell}=\alpha$, i.e., as soon as

$$
L_{\mu, s_{q}, s_{q+\ell}}\left(\pi_{1}^{-1}\left(A_{s}\right)\right)=L_{\mu, s_{q}, \alpha}\left(\pi_{1}^{-1}\left(A_{s}\right)\right),
$$

which occurs as soon as $\ell \geq c_{7}^{-1} \int_{s_{q}}^{\alpha} \mu\left(A_{s}, \lambda_{s}, v_{s}\right)\left\|\left(\dot{A}_{s}, \dot{\lambda}_{s}, \dot{v}_{s}\right)\right\| d s$, as claimed in the theorem (note that $C:=c_{7}^{-1} \leq 3000$ ).

We finally prove Proposition 5.8. From Proposition 5.7 we have

$$
\begin{aligned}
& \mu(B, \zeta, w)^{2} \Delta s \underset{(31)}{\geq}\left(1-4 \sqrt{3} c_{4}\right)^{2} \int_{0}^{\Delta s} \mu\left(A_{s}, \lambda_{s}, v_{s}\right)^{2} d s \\
& \underset{\text { Prop. } 2.7}{\geq} \frac{\left(1-4 \sqrt{3} c_{4}\right)^{2}}{6} L_{\mu, 0, \Delta_{s}}\left(\pi_{1}^{-1}\left(A_{s}\right)\right) \geq \frac{c_{7}\left(1-4 \sqrt{3} c_{4}\right)^{2}}{6},
\end{aligned}
$$

so Proposition 5.8 follows.

\section{Integration in the solution variety}

\subsection{The coarea formula}

On a Riemannian manifold $M$ there is a well defined measure vol $_{M}$ obtained by integrating the indicator functions $\mathbb{1}_{A}$ of Borel measurable subsets $A \subseteq M$ against the volume form $d M$ of $M$,

$$
\operatorname{vol}_{M}(A):=\int_{M} \mathbb{1}_{A} d M .
$$

Dividing $\mathbb{1}_{A}$ by vol $M(M)$ if $\operatorname{vol}_{M}(M)<\infty$ leads to a natural notion of uniform distribution on $M$, which we will denote by $\mathscr{U}(M)$. More generally, we will call any measurable function $f: M \rightarrow[0, \infty]$ such that $\int_{M} f d M=1$ a probability density on $M$. We abuse notation and sometimes write $\mathbb{E}_{x \in M}$ instead of $\mathbb{E}_{x \sim \mathscr{U}(M)}$. 
The coarea formula (a modern classical formula due to Federer [28], see the Appendix of [30] for a smooth version) is an extension of the transformation formula to not necessarily bijective smooth maps between Riemannian manifolds. In order to state it, we first need to generalize the notion of Jacobians.

Suppose that $M, N$ are Riemannian manifolds of dimensions $m, n$, respectively, such that $m \geq n$. Let $\psi: M \rightarrow N$ be a smooth map. By definition, the derivative $D \psi(x): T_{x} M \rightarrow T_{\psi(x)} N$ at a regular point $x \in M$ is surjective. Hence the restriction of $D \psi(x)$ to the orthogonal complement of its kernel yields a linear isomorphism. The absolute value of its determinant is called the normal Jacobian (sometimes called normal determinant in the context of linear algebra [1]) of $\psi$ at $x$ and is denoted by $\mathrm{NJ} \psi(x)$. We set $\mathrm{NJ} \psi(x):=0$ if $x$ is not a regular point.

If $y$ is a regular value of $\psi$, then the fiber $F_{y}:=\psi^{-1}(y)$ is a Riemannian submanifold of $M$ of dimension $m-n$, and it makes sense to integrate functions on $F_{y}$. Moreover, Sard's lemma states that almost all $y \in N$ are regular values.

We can now state the coarea formula.

Theorem 6.1 (Coarea formula). Suppose that $M, N$ are Riemannian manifolds of dimensions $m, n$, respectively, and let $\psi: M \rightarrow N$ be a surjective smooth map such that $D \psi$ is surjective a.e. Let $F_{y}=\psi^{-1}(y)$. Then for any function $\chi: M \rightarrow \mathbb{R}$ that is integrable with respect to the volume measure of $M$ we have

$$
\int_{M} \chi d M=\int_{y \in N}\left(\int_{F_{y}} \frac{\chi}{\mathrm{NJ} \psi} d F_{y}\right) d N,
$$

and the integrals involved are well defined.

It should be clear that this result contains the change of variables formula as a special case. Moreover, if we apply the coarea formula to the projection $\pi_{2}: M \times N \rightarrow N$, $(x, y) \mapsto y$, we retrieve Fubini's theorem since $\mathrm{NJ} \pi_{2}=1$.

\subsection{Coarea formula and double fibrations}

The coarea formula can be readily applied to the following situation. Assume that three Riemannian manifolds $M, N_{1}, N_{2}$ are equipped with surjective smooth mappings $\pi_{1}: M \rightarrow N_{1}$ and $\pi_{2}: M \rightarrow N_{2}$ whose derivatives are a.e. surjective, so NJ $\pi_{1}$ and $\mathrm{NJ} \pi_{2}$ are a.e. nonzero. Let $\chi: M \rightarrow[0, \infty)$ be a measurable mapping. From Theorem 6.1 applied to $\pi_{1}$ we have (here $d x$ and $d y$ stand for the volume forms in $M$ and $N_{1}$, respectively)

$$
\int_{x \in M} \chi(x) \mathrm{NJ} \pi_{1}(x) d x=\int_{y \in N_{1}} \int_{x \in \pi_{1}^{-1}(y)} \chi(x) d x d y .
$$

On the other hand, Theorem 6.1 applied to $\pi_{2}$ yields

$$
\int_{x \in M} \chi(x) \mathrm{NJ} \pi_{1}(x) d x=\int_{z \in N_{2}} \int_{x \in \pi_{2}^{-1}(z)} \frac{\mathrm{NJ} \pi_{1}(x)}{\mathrm{NJ} \pi_{2}(x)} \chi(x) d x d z .
$$

We thus have the following result. 
Theorem 6.2. Let $M, N_{1}, N_{2}$ be Riemannian manifolds equipped with surjective smooth mappings $\pi_{1}: M \rightarrow N_{1}$ and $\pi_{2}: M \rightarrow N_{2}$ whose derivatives are a.e. surjective. Let $\chi: M \rightarrow[0, \infty)$ be a measurable mapping. Then

$$
\int_{y \in N_{1}} \int_{x \in \pi_{1}^{-1}(y)} \chi(x) d x d y=\int_{z \in N_{2}} \int_{x \in \pi_{2}^{-1}(z)} \frac{\mathrm{NJ} \pi_{1}(x)}{\mathrm{NJ} \pi_{2}(x)} \chi(x) d x d z .
$$

(Note that when $M$ and $N_{1}$ have the same dimension, one can replace the inner integral on the left-hand side by a (finite or countable) sum.)

In the next sections we shall apply this result in two different contexts. A linear algebra argument simplifies the computation of the quotient of normal Jacobians. Let $E$ and $F$ be finite-dimensional, complex Euclidean vector spaces and let $\varphi: E \rightarrow F$ be a surjective linear mapping. Consider the graph $\Gamma:=\{(x, \varphi(x)) \mid x \in E\}$ of $\varphi$. Then $\Gamma$ is a linear subspace of $E \times F$ and the two projections

$$
p_{1}: \Gamma \rightarrow E,(x, \varphi(x)) \mapsto x, \quad \text { and } \quad p_{2}: \Gamma \rightarrow F,(x, \varphi(x)) \mapsto \varphi(x),
$$

are linear maps. Note that $p_{1}$ is an isomorphism and $p_{2}$ is surjective as $\varphi$ is.

Lemma 6.3. Under the above assumptions, we have

$$
\frac{\mathrm{NJ} p_{1}}{\mathrm{NJ} p_{2}}=\left|\operatorname{det}\left(\varphi \varphi^{*}\right)\right|^{-1}
$$

Proof. This result is [14, Lemma 3(b), p. 242], although we rewrite it for complex vector spaces here (note the comment in [14, proof of Theorem 5, p. 243]).

\subsection{The solution variety for the eigenpair problem}

Recall from $\$ 2.2$ that we have the two projections

$$
\pi_{1}: \mathcal{V} \rightarrow \mathbb{C}^{n \times n},(A, \lambda, v) \rightarrow A, \quad \text { and } \quad \pi_{2}: \mathcal{V} \rightarrow \mathbb{C} \times \mathbb{P}\left(\mathbb{C}^{n}\right),(A, \lambda, v) \rightarrow(\lambda, v),
$$

and, for $(A, \lambda, v) \in \mathcal{V}$, the linear operator $A_{\lambda, v}: v^{\perp} \rightarrow v^{\perp}$ given by $P_{v^{\perp}}(A-\lambda \text { Id })_{\mid v^{\perp}}$. In order to apply Theorem 6.2 we first need to compute the quotient of normal Jacobians there.

Proposition 6.4. Let $p:=(A, \lambda, v) \in \mathcal{W}$ and choose a representative such that $\|v\|=1$. Then the derivative $D \pi_{1}(p): T_{p} \mathcal{W} \rightarrow T_{A} \mathbb{C}^{n \times n}$ is an isomorphism, the derivative $D \pi_{2}(p): T_{p} \mathcal{W} \rightarrow T_{(\lambda, v)}\left(\mathbb{C} \times \mathbb{P}\left(\mathbb{C}^{n}\right)\right)$ is surjective, and

$$
\frac{\mathrm{NJ} \pi_{1}(p)}{\mathrm{NJ} \pi_{2}(p)}=\left|\operatorname{det}\left(A_{\lambda, v}\right)\right|^{2}=\operatorname{det}\left(A_{\lambda, v} A_{\lambda, v}^{*}\right) .
$$

Proof. By unitary invariance, we may assume without loss of generality that $v=e_{1}=$ $(1,0, \ldots, 0)$, and then

$$
A=\left(\begin{array}{cc}
\lambda & c^{*} \\
0 & B
\end{array}\right), \quad c \in \mathbb{C}^{n-1}, B \in \mathbb{C}^{(n-1) \times(n-1)}
$$


so $A_{\lambda, v}=B-\lambda \operatorname{ld}_{n-1}$. Let $\Gamma=\left\{(\dot{A}, D G(A) \dot{A}): \dot{A} \in \mathbb{C}^{n \times n}\right\} \subseteq \mathbb{C}^{n \times n} \times \mathbb{C} \times v^{\perp}$ where $G$ is the appropriate branch of the solution map defined in some open neighborhood of $A$. We are under the hypotheses of Lemma 6.3, so

$$
\frac{\mathrm{NJ} \pi_{1}(p)}{\mathrm{NJ} \pi_{2}(p)}=\frac{\mathrm{NJ}\left(D \pi_{1}(p)\right)}{\mathrm{NJ}\left(D \pi_{2}(p)\right)}=\operatorname{det}\left(D G(A, \lambda, v) D G(A, \lambda, v)^{*}\right)^{-1} .
$$

From Lemmas 3.1 and 3.2, we have

$$
D G(A, \lambda, v) \dot{A}=\left(\begin{array}{c}
\left\langle\dot{A} v, v-i_{\mathbb{C}^{n}} A_{\lambda, v}^{-*} P_{v^{\perp}} A^{*} v\right\rangle \\
-A_{\lambda, v}^{-1} P_{v^{\perp}} \dot{A} v
\end{array}\right)=\left(\begin{array}{c}
v^{*}-v^{*} A i_{\mathbb{C}^{n}} A_{\lambda, v}^{-1} P_{v^{\perp}} \\
-A_{\lambda, v}^{-1} P_{v^{\perp}}
\end{array}\right) \dot{A} v=R \dot{A} v,
$$

where $R: \mathbb{C}^{n} \rightarrow \mathbb{C} \times v^{\perp} \equiv \mathbb{C}^{n}$ is the linear operator multiplying by $\dot{A} v$ in the last formula. A standard linear algebra argument then shows that $\operatorname{det}\left(D G(A, \lambda, v) D G(A, \lambda, v)^{*}\right)=$ $\operatorname{det}\left(R R^{*}\right)=|\operatorname{det}(R)|^{2}$. Now, we can identify

$$
i_{\mathbb{C}^{n}} \equiv\left(\begin{array}{c}
0 \\
\operatorname{ld}_{n-1}
\end{array}\right), \quad P_{v^{\perp}} \equiv\left(\begin{array}{ll}
0 & \mathrm{Id}_{n-1}
\end{array}\right),
$$

which implies that in the standard basis we have

$$
R=\left(\begin{array}{cc}
1 & * \\
0 & -A_{\lambda, v}^{-1}
\end{array}\right)
$$

thus showing that $|\operatorname{det}(R)|^{2}=\left|\operatorname{det}\left(A_{\lambda, v}^{-1}\right)\right|^{2}$, and the proposition follows.

We are now ready to rewrite Theorem 6.2 in this setting. The following is an important technical result that we will use several times.

Proposition 6.5. Let $\chi: \mathcal{V} \rightarrow[0, \infty)$ be a measurable mapping. Then

$$
\begin{aligned}
\int_{A \in \mathbb{C}^{n \times n}} \sum_{\lambda, v: A v=\lambda v} \chi(A, \lambda, v) d A \\
=\int_{(\lambda, v) \in \mathbb{C} \times \mathbb{P}\left(\mathbb{C}^{n}\right)} \int_{A: A v=\lambda v} \chi(A, \lambda, v)\left|\operatorname{det}\left(A_{\lambda, v}\right)\right|^{2} d A d(\lambda, v) .
\end{aligned}
$$

Moreover, assume that $\chi$ is unitarily invariant in the sense that $\chi(A, \lambda, v)=$ $\chi\left(U A U^{*}, \lambda, U v\right)$ for any unitary matrix $U \in \mathcal{U}_{n}$. Fix any a.e. continuous mapping $\mathbb{C}^{n} \backslash\{0\} \rightarrow \mathcal{U}_{n}, v \mapsto U_{v}$, such that $U_{v} e_{1}=v /\|v\|$ for all $v$. Then, for every $\hat{A} \in \mathbb{C}^{n \times n}$ and $\sigma>0$,

$$
\begin{aligned}
& \underset{A \sim \mathcal{N}_{\mathbb{C}^{n \times n}\left(\hat{A}, \sigma^{2}\right)}}{\mathbb{E}} \sum_{\lambda, v: A v=\lambda v} \chi(A, \lambda, v) \\
& =\frac{1}{\Gamma(n) \sigma^{2(n-1)}} \underset{v}{\mathbb{E}} \underset{(\lambda, w, B)}{\mathbb{E}} e^{-\left\|\hat{y}_{v}\right\|^{2} / \sigma^{2}} \chi\left(\left(\begin{array}{cc}
\lambda & w^{*} \\
0 & B
\end{array}\right), \lambda, e_{1}\right)\left|\operatorname{det}\left(B-\lambda I_{n-1}\right)\right|^{2},
\end{aligned}
$$

where $v \in \mathbb{P}\left(\mathbb{C}^{n}\right)$ has the uniform distribution, $\hat{y}_{v}=P_{v^{\perp}} \hat{A} v /\|v\|$, and $\lambda \sim \mathcal{N}_{\mathbb{C}}\left(\hat{\lambda}, \sigma^{2}\right)$, $w \sim \mathcal{N}_{\mathbb{C}^{n-1}}\left(\hat{w}, \sigma^{2}\right)$, and $B \sim \mathcal{N}_{\mathbb{C}^{(n-1) \times(n-1)}}\left(\hat{B}, \sigma^{2}\right)$ are independent Gaussian random 
variables centered at

$$
\hat{\lambda}:=\frac{\langle\hat{A} v, v\rangle}{\|v\|^{2}}, \quad \hat{w}:=J^{*} U_{v}^{*} \hat{A}^{*} U_{v} e_{1}, \quad \hat{B}:=J^{*} U_{v}^{*} \hat{A} U_{v} J .
$$

Here, $J$ is the $n \times(n-1)$ matrix whose columns are $e_{2}, \ldots, e_{n}\left(\right.$ and hence $J^{*}=\left(0 \mathrm{Id}_{n-1}\right)$ is the matrix of $\left.P_{e_{1}^{\perp}}: \mathbb{C}^{n} \rightarrow e_{1}^{\perp}\right)$. In particular, if $\hat{A}=0$ then $\hat{y}_{v}=0, \hat{\lambda}=0, \hat{w}=0$ and $\hat{B}=0$, so $v$ can be removed from the expected value in (38).

Proof. The first claim follows directly from Theorem 6.2 and Proposition 6.4.

For the second claim, let $I$ be the left-hand side of (38). Change $\chi(A, \lambda, v)$ to $\left(\sigma^{2} \pi\right)^{-n^{2}} \chi(A, \lambda, v) e^{-\|A-\hat{A}\|_{F}^{2} / \sigma^{2}}$ in the first formula to get

$$
I=\frac{1}{\left(\sigma^{2} \pi\right)^{n^{2}}} \int_{(\lambda, v) \in \mathbb{C} \times \mathbb{P}\left(\mathbb{C}^{n}\right)} \int_{A: A v=\lambda v} \chi(A, \lambda, v)\left|\operatorname{det}\left(A_{\lambda, v}\right)\right|^{2} e^{-\|A-\hat{A}\|_{F}^{2} / \sigma^{2}} d A d(\lambda, v) .
$$

Note that $\{A: A v=\lambda v\}$ can be parametrized by

$$
(w, B) \mapsto A=U_{v}\left(\begin{array}{cc}
\lambda & w^{*} \\
0 & B
\end{array}\right) U_{v}^{*},
$$

where $w \in \mathbb{C}^{n-1}$ and $B \in \mathbb{C}^{(n-1) \times(n-1)}$. This parametrization preserves distances; moreover $\left|\operatorname{det}\left(A_{\lambda, v}\right)\right|=\left|\operatorname{det}\left(\lambda I_{n-1}-B\right)\right|$ and from the fact that $\chi$ is unitarily invariant, we have

$$
\begin{aligned}
& I= \\
& \frac{1}{\left(\sigma^{2} \pi\right)^{n^{2}}} \int_{(\lambda, v, w, B)} \chi\left(\left(\begin{array}{cc}
\lambda & w^{*} \\
0 & B
\end{array}\right), \lambda, e_{1}\right)\left|\operatorname{det}\left(\lambda I_{n-1}-B\right)\right|^{2} e^{-\|A-\hat{A}\|_{F}^{2} / \sigma^{2}} d(\lambda, v, w, B),
\end{aligned}
$$

where $A$ is given by the formula above. Note now that we can write

$$
\begin{aligned}
\|A-\hat{A}\|_{F}^{2}= & \left\|\left(\begin{array}{cc}
\lambda & w^{*} \\
0 & B
\end{array}\right)-U_{v}^{*} \hat{A} U_{v}\right\|_{F}^{2} \\
= & \left|\lambda-e_{1}^{*} U_{v}^{*} \hat{A} U_{v} e_{1}\right|^{2}+\left\|w^{*}-e_{1}^{*} U_{v}^{*} \hat{A} U_{v} J\right\|^{2}+\left\|B-J^{*} U_{v}^{*} \hat{A} U_{v} J\right\|_{F}^{2} \\
& +\left\|J^{*} U_{v}^{*} \hat{A} U_{v} e_{1}\right\|^{2} \\
= & \left|\lambda-\frac{v^{*} \hat{A} v}{\|v\|^{2}}\right|^{2}+\left\|w-J^{*} U_{v}^{*} \hat{A}^{*} U_{v} e_{1}\right\|^{2}+\left\|B-J^{*} U_{v}^{*} \hat{A} U_{v} J\right\|_{F}^{2}+\left\|\hat{y}_{v}\right\|^{2},
\end{aligned}
$$

and the second claim of the theorem follows if we note that the volume of $\mathbb{P}\left(\mathbb{C}^{n}\right)$ is $\pi^{n-1} / \Gamma(n)$.

\subsection{The linear solution variety}

It will be useful to consider a geometrical scheme similar to that of $\S 6.3$ for the case of solving linear systems: we consider

$$
\mathcal{V}^{\text {lin }}:=\left\{(M, v) \in \mathbb{C}^{(n-1) \times n} \times \mathbb{P}\left(\mathbb{C}^{n}\right): M v=0\right\} .
$$


The linear solution variety $\mathcal{V}^{\text {lin }}$ is an $n(n-1)$-dimensional smooth submanifold of $\mathbb{C}^{(n-1) \times n} \times \mathbb{P}\left(\mathbb{C}^{n}\right)$, and again it inherits the Riemannian structure of the ambient space (cf. [19, (17.14)]).

The linear solution variety is equipped with two projections

$$
\pi_{1}^{\operatorname{lin}}: \mathcal{V}^{\text {lin }} \rightarrow \mathbb{C}^{(n-1) \times n},(M, v) \mapsto M, \quad \pi_{2}^{\operatorname{lin}}: \mathcal{V}^{\text {lin }} \rightarrow \mathbb{P}\left(\mathbb{C}^{n}\right),(M, v) \mapsto v .
$$

Note that $\pi_{2}^{\text {lin }}$ is regular at every $(M, v) \in \mathcal{V}^{\text {lin }}$ and $\pi_{1}^{\operatorname{lin}}$ is regular at $(M, v) \in \mathcal{V}^{\text {lin }}$ if and only if $M$ is of maximal rank.

For $M \in \mathbb{C}^{(n-1) \times n},\left(\pi^{\text {lin }}\right)^{-1}(M)$ is a copy of the projective linear subspace corresponding to the kernel of $M$ in $\mathbb{P}\left(\mathbb{C}^{n}\right)$, and for $v \in \mathbb{P}\left(\mathbb{C}^{n}\right),\left(\pi_{2}^{\text {lin }}\right)^{-1}(v)$ is a copy of the linear subspace of $\mathbb{C}^{(n-1) \times n}$ consisting of the matrices $A$ such that $A v=0$.

We can apply Theorem 6.2 to integrate functions in $\mathcal{V}^{\text {lin }}$ using the projections in (39). The tangent space to $\mathcal{V}^{\text {lin }}$ at a regular point $(M, v)$ can be identified with

$$
\left\{(\dot{M}, \dot{v}): \dot{M} v+M \dot{v}=0, v^{*} \dot{v}=0\right\}=\{(\dot{M}, \dot{v}): \dot{v}=\varphi(\dot{M})\}, \quad \varphi(\dot{M})=-M^{\dagger} \dot{M} v
$$

Note that $\varphi$ is a linear mapping defined from $\mathbb{C}^{(n-1) \times n}$ to $v^{\perp}$. A routine computation shows that, if $\|v\|=1$, then $\varphi \varphi^{*}: v^{\perp} \rightarrow v^{\perp}$ satisfies $\varphi \varphi^{*}(w)=P_{v^{\perp}} M^{\dagger}\left(M^{\dagger}\right)^{*} i_{\mathbb{C}^{n}} w$. Writing down the singular value decomposition of $M$, it follows that $\operatorname{det}\left(\varphi \varphi^{*}\right)=$ $\operatorname{det}\left(M M^{*}\right)^{-1}$. From Lemma 6.3 it follows that

$$
\frac{\mathrm{NJ}\left(\pi^{\operatorname{lin}}\right)(M, v)}{\mathrm{NJ}\left(\pi_{2}^{\operatorname{lin}}\right)(M, v)}=\left|\operatorname{det}\left(M M^{*}\right)\right| .
$$

Proposition 6.6. Let $\phi^{\mathrm{lin}}: \mathcal{V}^{\mathrm{lin}} \rightarrow[0, \infty]$ be a measurable unitarily invariant function in the sense that $\phi^{\operatorname{lin}}(M, v)=\phi^{\operatorname{lin}}\left(M U^{*}, U v\right)$ for any unitary matrix $U \in \mathcal{U}_{n}$. Then

$$
\underset{M \sim \mathcal{N}_{\mathbb{C}^{(n-1) \times n}}}{\mathbb{E}}\left(\phi^{\operatorname{lin}}(M, \operatorname{ker}(M))=\frac{1}{\Gamma(n)} \underset{B \sim \mathcal{N}_{\mathbb{C}^{(n-1) \times(n-1)}}}{\mathbb{E}} \phi^{\operatorname{lin}}\left((0 B), e_{1}\right)|\operatorname{det}(B)|^{2} .\right.
$$

Proof. Let $\chi(M)=\phi^{\operatorname{lin}}(M, \operatorname{ker}(M)) e^{-\|M\|_{F}^{2}}$. Theorem 6.2 and (40) imply that

$$
\int_{M \in \mathbb{C}^{(n-1) \times n}} \chi(M) d M=\int_{v \in \mathbb{P}\left(\mathbb{C}^{n}\right)} \int_{M: M v=0}\left|\operatorname{det}\left(M M^{*}\right)\right| \chi(M) d M d v .
$$

Now, $\left|\operatorname{det}\left(M M^{*}\right)\right|^{2} \chi(M)=\left|\operatorname{det}\left(M U(M U)^{*}\right)\right|^{2} \chi\left(M U^{*}\right)$ for all $U \in \mathcal{U}_{n}$ by hypothesis. Hence, by parametrizing $\{M: M v=0\}$ by $\left\{\left(\begin{array}{ll}0 & B\end{array}\right) U_{v}^{*}: B \in \mathbb{C}^{(n-1) \times(n-1)}\right\}$ where $U_{v} \in \mathcal{U}_{n}$ is any matrix satisfying $U_{v} e_{1}=v$ (we are assuming $\|v\|=1$ ), we conclude that the inner integral on the right-hand side above does not depend on $v$. We thus have

$$
\int_{M \in \mathbb{C}^{(n-1) \times n}} \chi(M) d M=\operatorname{vol}\left(\mathbb{P}\left(\mathbb{C}^{n}\right)\right) \int_{B \in \mathbb{C}^{(n-1) \times(n-1)}}|\operatorname{det}(B)|^{2} \chi((0, B)) d B .
$$

The proposition follows from the form of the Gaussian density (recall $§ 2.6$ ) by noting that $\operatorname{vol}\left(\mathbb{P}\left(\mathbb{C}^{n}\right)\right)=\pi^{n-1} / \Gamma(n)$. 
Assume now that we are given an a.e. continuous function $\alpha: \mathbb{C}^{(n-1) \times(n-1)} \rightarrow[0, \infty]$. We can produce a unitarily invariant function defined on $\mathcal{V}^{\text {lin }}$ as follows:

$$
\phi^{\operatorname{lin}}(M, v)=\underset{Q:(M, Q) \in \mathcal{A}_{n}}{\mathbb{E}} \alpha(M Q),
$$

where $\mathcal{A}_{n}$ is given in (22). Note that

$$
\phi^{\operatorname{lin}}\left((0 B), e_{1}\right)=\underset{U \in \mathcal{U}_{n-1}}{\mathbb{E}} \alpha(B U) .
$$

It is a simple exercise to check that $\phi^{\text {lin }}$ is unitarily invariant in the sense of Proposition 6.6. Applying Proposition 6.6 to $\phi^{\text {lin }}$ then yields

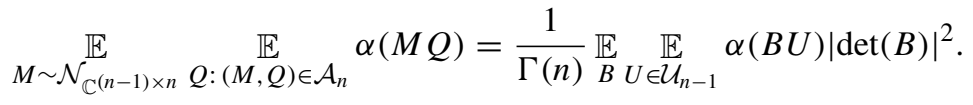

(The expected value in the unitary group is again with respect to the uniform distribution coming from its Riemannian structure.) Finally, using Fubini's theorem, we can interchange the integration order in the right-hand term, and then note that the isometry $B \mapsto B U$ preserves the value of the integral inside. We obtain the following corollary.

Corollary 6.7. Let $\alpha: \mathbb{C}^{(n-1) \times(n-1)} \rightarrow[0, \infty]$ be an a.e. continuous function. Then

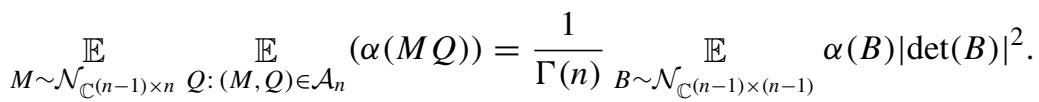

\section{Proof of Theorem 2.14}

We begin with the following result.

Proposition 7.1. The following inequality holds for every $\hat{A} \in \mathbb{C}^{m \times m}$ and $\sigma>0$ :

$$
\underset{A \sim \mathcal{N}_{\mathbb{C}^{m \times m}}\left(\hat{A}, \sigma^{2}\right)}{\mathbb{E}}\left\|A^{-1}\right\|_{F}^{2}|\operatorname{det}(A)|^{2} \leq \frac{m}{\sigma^{2}} \underset{A \sim \mathcal{N}_{\mathbb{C}^{m \times m}}\left(\hat{A}, \sigma^{2}\right)}{\mathbb{E}}|\operatorname{det}(A)|^{2} .
$$

Furthermore, equality holds if and only if $\hat{A}=0$. In particular,

$$
\underset{A \sim \mathcal{N}_{\mathbb{C}^{m \times m}}\left(0, \sigma^{2}\right)}{\mathbb{E}}\left\|A^{-1}\right\|_{F}^{2}|\operatorname{det}(A)|^{2}=m ! m \sigma^{2 m-2} .
$$

Proof. Expanding the determinant of $A$ by the $k$ th column we have

$$
\operatorname{det}(A)=\sum_{j=1}^{m}(-1)^{j+k} a_{j, k} \operatorname{det}\left(A^{j, k}\right),
$$

where $A^{j, k}$ denotes the matrix that results from $A$ by removing the $j$ th row and $k$ th column. Hence,

$$
|\operatorname{det}(A)|^{2}=\operatorname{det}(A) \overline{\operatorname{det}(A)}=\sum_{j, j^{\prime}=1}^{m}(-1)^{j+j^{\prime}+2 k} a_{j, k} \overline{a_{j^{\prime}, k}} \operatorname{det}\left(A^{j, k}\right) \overline{\operatorname{det}\left(A^{j^{\prime}, k}\right)} .
$$


Observe that the random variables $a_{j, k}$ and $a_{j^{\prime}, k}$ are independent of $\operatorname{det}\left(A^{j, k}\right)$ and $\operatorname{det}\left(A^{j^{\prime}, k}\right)$. Then

$$
\underset{A \sim \mathcal{N}_{\mathbb{C}^{m \times m}}\left(\hat{A}, \sigma^{2}\right)}{\mathbb{E}}|\operatorname{det}(A)|^{2}=\sum_{j, j^{\prime}=1}^{m}(-1)^{j+j^{\prime}+2 k} \mathbb{E}\left(a_{j, k} \overline{a_{j^{\prime}, k}}\right) \mathbb{E}\left(\operatorname{det}\left(A^{j, k}\right) \overline{\operatorname{det}\left(A^{j^{\prime}, k}\right)}\right) .
$$

Now observe that

$$
\mathbb{E}\left(a_{j, k} \overline{a_{j^{\prime}, k}}\right)= \begin{cases}\widehat{a}_{j, k} \overline{\widehat{a}_{j^{\prime}, k}} & \text { if } j \neq j^{\prime}, \\ \sigma^{2}+\left|\widehat{a}_{j, k}\right|^{2} & \text { otherwise. }\end{cases}
$$

We conclude that for $k=1, \ldots, m$,

$$
\underset{A \sim \mathcal{N}_{\mathbb{C}^{m} \times m}\left(\hat{A}, \sigma^{2}\right)}{\mathbb{E}}|\operatorname{det}(A)|^{2}=\mathbb{E}\left|\operatorname{det}\left(\left[A ; k ; \hat{A}_{k}\right]\right)\right|^{2}+\sigma^{2} \sum_{j=1}^{m} \mathbb{E}\left|\operatorname{det} A^{j, k}\right|^{2},
$$

where $\left[A ; k ; \hat{A}_{k}\right]$ is the matrix formed by replacing the (random) $k$ th column of $A$ by the (deterministic) $k$ th column of $\hat{A}$. Summing on $k$ we get

$$
m \underset{A}{\mathbb{E}}|\operatorname{det}(A)|^{2}=\sum_{k=1}^{m} \mathbb{E}\left|\operatorname{det}\left(\left[A ; k ; \hat{A}_{k}\right]\right)\right|^{2}+\sigma^{2} \sum_{j, k=1}^{m} \mathbb{E}\left|\operatorname{det} A^{j, k}\right|^{2} .
$$

On the other hand, from a direct application of Cramer's rule and (42), we deduce that

$$
\begin{aligned}
\sigma^{2} \underset{A \sim \mathcal{N}_{\mathbb{C}^{m \times m}}\left(\hat{A}, \sigma^{2}\right)}{\mathbb{E}}\left\|A^{-1}\right\|_{F}^{2}|\operatorname{det}(A)|^{2} & =\sigma^{2} \sum_{j, k=1}^{m} \mathbb{E}\left|\operatorname{det} A^{j, k}\right|^{2} \\
& =m \mathbb{E}|\operatorname{det}(A)|^{2}-\sum_{k=1}^{m} \mathbb{E}\left|\operatorname{det}\left(\left[A ; k ; \hat{A}_{k}\right]\right)\right|^{2},
\end{aligned}
$$

and the first claim of the proposition follows. Moreover, when $\hat{A}=0$, the last term in the sum above is zero. We leave the proof of the converse to the reader. Using (41) and the fact that the matrices $A^{j, k}$ are $\mathcal{N}_{\mathbb{C}^{(m-1) \times(m-1)}}\left(0, \sigma^{2}\right)$-distributed, one can prove working by induction the equality

$$
\underset{A \sim \mathcal{N}_{\mathbb{C}^{m \times m}}\left(0, \sigma^{2}\right)}{\mathbb{E}}|\operatorname{det}(A)|^{2}=\sigma^{2 m} m !
$$

The second claim of the proposition follows.

Corollary 7.2.

$$
\underset{M \sim \mathcal{N}_{\mathbb{C}^{(n-1) \times n}}^{\mathbb{E}}}{\mathbb{E}}\left\|M^{\dagger}\right\|_{F}^{2}=n-1 .
$$

Proof. From Proposition 6.6 with $\phi^{\operatorname{lin}}(M, \zeta)=\left\|M^{\dagger}\right\|_{F}^{2}$, we have

$$
\underset{M \sim \mathcal{N}_{\mathbb{C}^{(n-1) \times n}}^{\mathbb{E}}}{\mathbb{E}}\left\|M^{\dagger}\right\|_{F}^{2}=\frac{1}{\Gamma(n)} \underset{B \sim \mathcal{N}_{\mathbb{C}^{(n-1) \times(n-1)}}}{\mathbb{E}}\left\|B^{-1}\right\|_{F}^{2}|\operatorname{det}(B)|^{2} \underset{\text { Prop. } 7.1}{=} n-1
$$

as claimed. 
Corollary 7.3. For any $\hat{B} \in \mathbb{C}^{(n-1) \times(n-1)}, \sigma>0$, and $\lambda \in \mathbb{C}$, we have

$$
\underset{B}{\mathbb{E}}\left\|\left(B-\lambda I_{n-1}\right)^{-1}\right\|_{F}^{2}\left|\operatorname{det}\left(B-\lambda I_{n-1}\right)\right|^{2} \leq \frac{n-1}{\sigma^{2}} \underset{B}{\mathbb{E}}\left|\operatorname{det}\left(B-\lambda I_{n-1}\right)\right|^{2},
$$

where $B \sim \mathcal{N}_{\mathbb{C}^{(n-1) \times(n-1)}}\left(\hat{B}, \sigma^{2}\right)$.

Proof. Note that

$$
\underset{B}{\mathbb{E}}\left\|\left(B-\lambda I_{n-1}\right)^{-1}\right\|_{F}^{2}\left|\operatorname{det}\left(B-\lambda I_{n-1}\right)\right|^{2}=\underset{C \sim \mathcal{N}_{\mathbb{C}^{(n-1) \times(n-1)}}\left(\widehat{C}, \sigma^{2}\right)}{\mathbb{E}}\left\|C^{-1}\right\|_{F}^{2}|\operatorname{det} C|^{2},
$$

where $\widehat{C}=\widehat{B}-\lambda I_{n-1}$. The proof readily follows from Proposition 7.1.

Proof of Theorem 2.14. Fix any a.e. continuous mapping $v \mapsto U_{v}$ such that for $v$ in $\mathbb{P}\left(\mathbb{C}^{n}\right), U_{v}$ is a unitary matrix with $U_{v} e_{0}=v /\|v\|$. From (38) applied to $\chi(A, \lambda, v)=$ $\frac{1}{n} \mu_{F}^{2}(A, \lambda, v) /\|A\|_{F}^{2}=\frac{1}{n}\left\|A_{\lambda, v}^{-1}\right\|_{F}^{2}$ we have

$$
\begin{aligned}
\underset{A \sim \mathcal{N}_{\mathbb{C}^{n \times n}}\left(\hat{A}, \sigma^{2}\right)}{\mathbb{E}} \frac{\mu_{F, \mathrm{av}}(A)^{2}}{\|A\|_{F}^{2}} \\
=\frac{1}{n \Gamma(n) \sigma^{2(n-1)}} \underset{v}{\mathbb{E}} \underset{(\lambda, w, B)}{\mathbb{E}} e^{-\left\|\hat{y}_{v}\right\|^{2} / \sigma^{2}}\left\|\left(B-\lambda I_{n-1}\right)^{-1}\right\|_{F}^{2}\left|\operatorname{det}\left(B-\lambda I_{n-1}\right)\right|^{2},
\end{aligned}
$$

where $\hat{y}_{v}=P_{v^{\perp}} \hat{A} v /\|v\|, v \in \mathbb{P}\left(\mathbb{C}^{n}\right)$, has the uniform distribution, and $\lambda \sim \mathcal{N}_{\mathbb{C}}\left(\hat{\lambda}, \sigma^{2}\right)$, $w \sim \mathcal{N}_{\mathbb{C}^{n-1}}\left(\hat{w}, \sigma^{2}\right)$, and $B \sim \mathcal{N}_{\mathbb{C}^{(n-1) \times(n-1)}}\left(\hat{B}, \sigma^{2}\right)$ for some $\hat{\lambda}, \hat{w}, \hat{B}$ which depend uniquely on $\hat{A}$ and $v$.

From (43) and Corollary 7.3 we have

$\underset{A \sim \mathcal{N}_{\mathbb{C}^{n \times n}}\left(\hat{A}, \sigma^{2}\right)}{\mathbb{E}} \frac{\mu_{F, \mathrm{av}}(A)^{2}}{\|A\|_{F}^{2}} \leq \frac{n-1}{\sigma^{2} \sigma^{2(n-1)} \Gamma(n+1)} \underset{v}{\mathbb{E}} \underset{(\lambda, w, B)}{\mathbb{E}} e^{-\left\|\hat{y}_{v}\right\|^{2} / \sigma^{2}}\left|\operatorname{det}\left(B-\lambda I_{n-1}\right)\right|^{2}$.

Now, if we apply again (38) to the constant function $\chi \equiv 1 / n$ we get

$$
1=\underset{A \sim \mathcal{N}_{\mathbb{C}^{m \times m}}\left(\hat{A}, \sigma^{2}\right)}{\mathbb{E}} 1=\frac{1}{\Gamma(n+1) \sigma^{2(n-1)}} \underset{v}{\mathbb{E}} \underset{(\lambda, w, B)}{\mathbb{E}} e^{-\left\|\hat{y}_{v}\right\|^{2} / \sigma^{2}}\left|\operatorname{det}\left(B-\lambda I_{n-1}\right)\right|^{2},
$$

and we have thus proved that

$$
\underset{A \sim \mathcal{N}_{\mathbb{C}^{n \times n}\left(\hat{A}, \sigma^{2}\right)}}{\mathbb{E}} \frac{\mu_{F, \mathrm{av}}(A)^{2}}{\|A\|_{F}^{2}} \leq \frac{n-1}{\sigma^{2}} \leq \frac{n}{\sigma^{2}},
$$

as claimed.

This proves the first part of Theorem 2.14. For the second part, let

$$
I:=\underset{A \sim \mathscr{U}(\mathbb{S})}{\mathbb{E}} \mu_{F, \mathrm{av}}(A)^{2}
$$

be the quantity we want to compute. From the first part of the theorem (with $\hat{A}=0$ and $\sigma=1$ ) we have

$$
\frac{1}{\pi^{n^{2}}} \int_{A \in \mathbb{C}^{n \times n}} \frac{\mu_{F, \mathrm{av}}(A)^{2}}{\|A\|_{F}^{2}} e^{-\|A\|_{F}^{2}} d A \leq n .
$$


On the other hand,

$$
\frac{1}{\pi^{n^{2}}} \int_{A \in \mathbb{C}^{n \times n}} \frac{\mu_{F, \mathrm{av}}(A)^{2}}{\|A\|_{F}^{2}} e^{-\|A\|_{F}^{2}} d A=\frac{1}{\pi^{n^{2}}} \int_{0}^{\infty} \frac{e^{-\rho^{2}}}{\rho^{2}} \int_{A:\|A\|_{F}=\rho} \mu_{F, \mathrm{av}}(A)^{2} d A d \rho .
$$

Now, because $\mu_{F, \text { av }}(A)$ is invariant under multiplication of $A$ by nonzero complex numbers, denoting $v_{\rho}=\operatorname{vol}\left(\left\{A:\|A\|_{F}=\rho\right\}\right)$, we have

$$
\frac{1}{v_{\rho}} \int_{A:\|A\|_{F}=\rho} \mu_{F, \text { av }}(A)^{2} d A=I, \quad 0<\rho<\infty .
$$

We deduce from (44)-(46) that

$$
\frac{I}{\pi^{n^{2}}} \int_{0}^{\infty} \frac{v_{\rho} e^{-\rho^{2}}}{\rho^{2}} d \rho \leq n
$$

Note now that

to conclude that

$$
v_{\rho}=\frac{2 \pi^{n^{2}}}{\Gamma\left(n^{2}\right)} \rho^{2 n^{2}-1}
$$

$$
I \leq \frac{n \Gamma\left(n^{2}\right)}{2 \int_{0}^{\infty} \rho^{2 n^{2}-3} e^{-\rho^{2}} d \rho}=\frac{n \Gamma\left(n^{2}\right)}{\Gamma\left(n^{2}-1\right)}=n\left(n^{2}-1\right) \leq n^{3} .
$$

The theorem follows.

\section{Proof of Propositions 2.22 and 2.24}

\subsection{Proof of Proposition 2.22}

First note that Path-follow starts by normalizing the input, so from (14) we can assume that $\left\|A_{0}\right\|_{F}=1$ and $A \sim \mathcal{N}_{\mathbb{C}^{n \times n}, T}(0,1)$ where $T=\sqrt{2} n$. From Remark 2.23, for integration purposes we can also assume that $\left(\mathcal{L}_{A_{0}, A} \backslash\left\{A_{0}\right\}\right) \cap \Sigma=\emptyset$. Corollary 2.21 with $q=1$ implies that

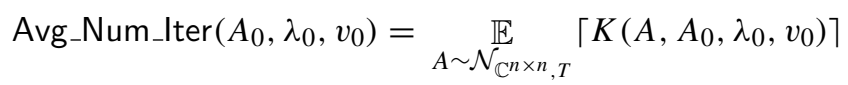

$$
\begin{aligned}
& \leq 2+\sqrt{6} C \underset{A \sim \mathcal{N}_{\mathbb{C}^{n \times n}, T}}{\mathbb{E}}\|A\|_{F} \int_{t_{1}}^{1} \frac{\mu\left(A_{t}, \lambda_{t}, v_{t}\right)^{2}}{\left\|A_{t}\right\|_{F}^{2}} d t \\
& \leq 2+\sqrt{6} C \underset{A \sim \mathcal{N}_{\mathbb{C}^{n \times n}, T}^{\mathbb{E}}}{\mathbb{E}}\|A\|_{F} \int_{t_{1}}^{1} \sum_{j=1}^{n} \frac{\mu\left(A_{t}, \lambda_{t}^{(j)}, v_{t}^{(j)}\right)^{2}}{\left\|A_{t}\right\|_{F}^{2}} d t,
\end{aligned}
$$

where $A_{t}=(1-t) A_{0}+t A$, the pairs $\left(\lambda_{t}^{(j)}, v_{t}^{(j)}\right)$ are defined by continuation for all the eigenpairs of $A_{t}$, and

$$
t_{1}=\inf _{\|A\|_{F} \leq T} \frac{1}{\|A\|_{F}\left(\sin \alpha \cot \left(\text { Choose_step }\left(A_{0}, \dot{A}_{0}, \lambda_{0}, v_{0}\right)\right)-\cos \alpha\right)+1} .
$$


We therefore have (use $T=\sqrt{2} n$ )

$$
\begin{aligned}
\operatorname{Avg\_ Num\_ Iter}\left(A_{0}, \lambda_{0}, v_{0}\right) & \leq 2+\sqrt{6} C n T \underset{A \sim \mathcal{N}_{\mathbb{C}^{n \times n}, T}}{\mathbb{E}} \int_{t_{1}}^{1} \frac{\mu_{\mathrm{av}}\left(A_{t}\right)^{2}}{\left\|A_{t}\right\|_{F}^{2}} d t \\
& \leq 2+\sqrt{48} C n^{2} \underset{A \sim \mathcal{N}_{\mathbb{C}^{n \times n}}}{\mathbb{E}} \int_{t_{1}}^{1} \frac{\mu_{\mathrm{av}}\left(A_{t}\right)^{2}}{\left\|A_{t}\right\|_{F}^{2}} d t
\end{aligned}
$$

We have used (13) and Lemma 2.16 for the last inequality. In order to bound the last term in the previous expression, we interchange the order of integration,

$$
\underset{A \sim \mathcal{N}_{\mathbb{C}^{n \times n}}}{\mathbb{E}} \int_{t_{1}}^{1} \frac{\mu_{\mathrm{av}}\left(A_{t}\right)^{2}}{\left\|A_{t}\right\|_{F}^{2}} d t=\int_{t_{1}}^{1}\left(\underset{A \sim \mathcal{N}_{\mathbb{C}^{n \times n}}}{\mathbb{E}} \frac{\mu_{\mathrm{av}}\left(A_{t}\right)^{2}}{\left\|A_{t}\right\|_{F}^{2}}\right) d t .
$$

Now, for fixed $t$, if $A \sim \mathcal{N}_{\mathbb{C}^{n \times n}}$ then $A_{t}=(1-t) A_{0}+t A$ satisfies $A_{t} \sim \mathcal{N}_{\mathbb{C}^{n \times n}}\left((1-t) A_{0}, t^{2}\right)$, and from Theorem 2.14 we have

$$
\underset{A \sim \sim \mathcal{N}_{\mathbb{C}^{n \times n}}}{\mathbb{E}} \frac{\mu_{\mathrm{av}}\left(A_{t}\right)^{2}}{\left\|A_{t}\right\|_{F}^{2}} \leq \frac{n}{t^{2}},
$$

which implies

$$
\underset{A \sim \mathcal{N}_{\mathbb{C}^{n \times n}}}{\mathbb{E}} \int_{t_{1}}^{1} \frac{\mu_{\mathrm{av}}\left(A_{t}\right)^{2}}{\left\|A_{t}\right\|_{F}^{2}} d t \leq \int_{t_{1}}^{1} \frac{n}{t^{2}} d t \leq \frac{n}{t_{1}} .
$$

We are thus left with the task of evaluating $t_{1}$. Note that $s_{1}=$ Choose_step $\left(A_{0}, \dot{A}_{0}, \lambda_{0}, v_{0}\right)$ (the length of the first step in the execution of Path-follow) is at least $R / \mu\left(A_{0}, \lambda_{0}, v_{0}\right)^{2}$ by Proposition 5.8. Hence $\cot s_{1} \leq 1 / s_{1} \leq \mu\left(A_{0}, \lambda_{0}, v_{0}\right)^{2} / R$ and it follows that

$$
t_{1} \geq \frac{1}{T\left(\cot s_{1}+1\right)+1} \geq \Omega\left(\frac{1}{n \mu\left(A_{0}, \lambda_{0}, v_{0}\right)^{2}}\right) .
$$

Putting together this bound and inequalities (48) and (49) we deduce the claimed bound for Avg_Num_Iter $\left(A_{0}, \lambda_{0}, v_{0}\right)$.

We next prove the smoothed analysis bounds. Reasoning as in (47) we see that the smoothed number of iterations Smd_Num_Iter $\left(A_{0}, \lambda_{0}, v_{0}, \sigma\right)$ is bounded by

$$
2+\sqrt{6} C \sup _{\hat{A} \in \mathbb{S} A \sim \mathcal{N}_{\mathbb{C}^{n \times n}, T}}^{\mathbb{E}} \underset{\left.A, \sigma^{2}\right)}{ } \sum_{j=1}^{n}\|A\|_{F} \int_{t_{1}}^{1} \frac{\mu\left(A_{t}, \lambda_{t}^{(j)}, v_{t}^{(j)}\right)^{2}}{\left\|A_{t}\right\|_{F}^{2}} d t .
$$

The rest of the argument is almost exactly as above, the only difference being the bound $\|A\|_{F} \leq T+\|\hat{A}\|_{F}=\sqrt{2} n+1$. 


\subsection{Proof of Proposition 2.24}

We are now following all the $n$ paths (each starting with a different eigenpair of $A_{0}$ ). Applying Corollary 2.21 with $q=1$ to each of them we obtain

$$
\begin{aligned}
& \text { Avg_Num_Iter }\left(A_{0}\right)=\underset{A \sim \mathcal{N}_{\mathbb{C}^{n \times n}, T}}{\mathbb{E}} \sum_{j=1}^{n} K\left(A, A_{0}, \lambda^{(j)}, v^{(j)}\right) \\
& \leq 2 n+\sqrt{6} C \underset{A \sim \mathcal{N}_{\mathbb{C}^{n \times n}, T}^{\mathbb{E}}}{\mathbb{E}}\|A\|_{F} \sum_{j=1}^{n} \int_{t_{1}^{(j)}}^{1} \frac{\mu\left(A_{t}, \lambda_{t}^{(j)}, v_{t}^{(j)}\right)^{2}}{\left\|A_{t}\right\|_{F}^{2}} d t \\
& \leq 2 n+\sqrt{6} C \underset{A \sim \mathcal{N}_{\mathbb{C}^{n \times n}, T}}{\mathbb{E}}\|A\|_{F} \int_{t_{1}^{*}}^{1} \sum_{j=1}^{n} \frac{\mu\left(A_{t}, \lambda_{t}^{(j)}, v_{t}^{(j)}\right)^{2}}{\left\|A_{t}\right\|_{F}^{2}} d t,
\end{aligned}
$$

where $t_{1}^{*}=\min \left\{t_{1}^{(1)}, \ldots, t_{1}^{(n)}\right\}$. We can now reason as in the preceding proof to deduce that

$$
\operatorname{Avg} \text { _Num_Iter }\left(A_{0}\right)=\mathcal{O}\left(n^{3} / t_{1}^{*}\right)
$$

as well as $t_{1}^{(j)} \geq \Omega\left(\frac{1}{n \mu\left(A_{0}, \lambda^{(j)}, v^{(j)}\right)^{2}}\right)$ for $j=1, \ldots, n$. It follows from these bounds that

$$
t_{1}^{*} \geq \Omega\left(\frac{1}{n \mu_{\max }\left(A_{0}\right)^{2}}\right) .
$$

The rest of the proof follows as in the preceding proposition.

\section{Proof of Theorem 2.29}

We begin with an auxiliary result. For simplicity, in what follows we write $\mathbb{S}:=\mathbb{S}\left(\mathbb{C}^{n \times n}\right)$. We also consider the manifold

$$
\mathcal{S}:=\left\{(A, \dot{A}) \in \mathbb{S} \times \mathbb{S}: \dot{A} \in T_{A} \mathbb{S}\right\}
$$

and denote by $\mathscr{U}(\mathcal{S})$ the normalized product distribution on it, that is, the probability distribution coming from the product structure. Given any measurable mapping $\phi: \mathcal{S} \rightarrow$ $[0, \infty]$, let

$$
I_{\phi}:=\underset{A_{0}, A \in \mathbb{S}}{\mathbb{E}} \int_{0}^{d_{\mathbb{S}}\left(A_{0}, A\right)} \phi\left(A_{s}, \dot{A}_{s}\right) d s,
$$

where, as usual, the $A_{s}$ are such that $\left\{A_{s}: 0 \leq s \leq d_{\mathbb{S}}\left(A_{0}, A\right)\right\}=\mathcal{L}_{A_{0}, A}$, and $\dot{A}_{s}$ is the unit tangent vector (in the direction of the parametrization) to $\mathcal{L}_{A_{0}, A}$ at $A_{s}$.

Lemma 9.1. For any measurable mapping $\phi: \mathbb{S} \times \mathbb{S} \rightarrow[0, \infty]$ we have

$$
I_{\phi}=\frac{\pi}{2} \underset{(A, \dot{A}) \sim \mathscr{U}(\mathcal{S})}{\mathbb{E}} \phi(A, \dot{A}) .
$$

Proof. We consider the manifold $\mathcal{R}:=\left\{\left(A_{0}, A, s\right) \in \mathbb{S} \times \mathbb{S} \times(0, \pi): s<d_{\mathbb{S}}\left(A_{0}, A\right)\right\}$ with the product structure and let

$$
\psi: \mathcal{R} \rightarrow \mathcal{S}, \quad\left(A_{0}, A, s\right) \mapsto\left(A_{s}, \dot{A}_{s}\right)
$$


We can then write $I_{\phi}=\operatorname{vol}(\mathbb{S})^{-2} \int_{\mathcal{R}} \phi \circ \psi$. Applying the coarea formula (Theorem 6.1) yields

$$
I_{\phi}=\frac{1}{\operatorname{vol}(\mathbb{S})^{2}} \int_{(A, \dot{A}) \in \mathcal{S}} \phi(A, \dot{A}) q(A, \dot{A}) d(A, \dot{A}),
$$

where

$$
q(A, \dot{A})=\int_{\left(B_{0}, B, s\right) \in \psi^{-1}(A, \dot{A})} \frac{1}{\mathrm{NJ}(\psi)\left(B_{0}, B, s\right)} d\left(B_{0}, B, s\right) .
$$

Our goal now is to prove that $q(A, \dot{A})$ is a constant (independent of $A, \dot{A}$ ). It will be useful to consider the two diagonal matrices $\Delta=E_{11}$ and $\dot{\Delta}=E_{22}$ where $E_{i j}$ denotes the standard basis of $\mathbb{C}^{n \times n}$. Note that $(\Delta, \dot{\Delta}) \in \mathcal{S}$.

We now fix $(A, \dot{A}) \in \mathcal{S}$. Let $\sigma: \mathbb{C}^{n \times n} \rightarrow \mathbb{C}^{n \times n}$ be an isometric change of basis such that $\sigma(A)=\Delta$ and $\sigma(\dot{A})=\dot{\Delta}$. Denoting $\sigma_{\mathcal{S}}=\sigma \times \sigma$ and $\sigma_{\mathcal{R}}=\sigma \times \sigma \times \operatorname{ld}_{\mathbb{R}}$, where $\operatorname{ld}_{\mathbb{R}}$ is the identity mapping in $\mathbb{R}$, it is easy to check by writing down the formula for $A_{s}$ that $\psi \circ \sigma_{\mathcal{R}}=\sigma_{\mathcal{S}} \circ \psi$. We are under the hypothesis of [14, Lemma 4, p. 244] (which holds for surjective maps in general, not only for projections), proving that

$$
\mathrm{NJ}(\psi)\left(\sigma_{\mathcal{R}}^{-1}\left(C_{0}, C, s\right)\right)=\mathrm{NJ}(\psi)\left(C_{0}, C, s\right), \quad \forall\left(C_{0}, C, s\right) \in \mathcal{R} .
$$

Moreover, the mapping $\left.\sigma_{\mathcal{R}}\right|_{\psi^{-1}(A, \dot{A})}$ is an isometry from $\psi^{-1}(A, \dot{A})$ to $\psi^{-1}(\Delta, \dot{\Delta})$, which from the change of variables theorem implies

$$
\begin{aligned}
q(A, \dot{A}) & =\int_{\left(B_{0}, B, s\right) \in \psi^{-1}(A, \dot{A})} \frac{1}{\mathrm{NJ}(\psi)\left(B_{0}, B, s\right)} d\left(B_{0}, B, s\right) \\
& =\int_{\left(B_{0}, B, s\right) \in \psi^{-1}(A, \dot{A})} \frac{1}{\mathrm{NJ}(\psi)\left(\sigma_{\mathcal{R}}^{-1}\left(B_{0}, B, s\right)\right)} d\left(B_{0}, B, s\right) \\
& =\int_{\left(B_{0}, B, s\right) \in \psi^{-1}(\Delta, \dot{\Delta})} \frac{1}{\mathrm{NJ}(\psi)\left(B_{0}, B, s\right)} d\left(B_{0}, B, s\right)=q(\Delta, \dot{\Delta}),
\end{aligned}
$$

which proves that $q(A, \dot{A})$ is equal to some constant $\hat{C}$.

Since this holds for any measurable function $\phi$, we can take the latter to be constant with value 1 to derive the value of $\hat{C}$. Then (53) becomes

$$
I_{\phi}=\frac{1}{\operatorname{vol}(\mathbb{S})^{2}} \int_{(A, \dot{A}) \in \mathcal{S}} \hat{C} d(A, \dot{A})=\frac{\operatorname{vol}(\mathcal{S})}{\operatorname{vol}(\mathbb{S})^{2}} \hat{C} .
$$

And it follows from (51) (always with $\phi \equiv 1$ ) that

$$
I_{\phi}=\underset{A, A_{0} \in \mathbb{S}}{\mathbb{E}} d_{\mathbb{S}}\left(A_{0}, A\right)
$$

Hence,

$$
\underset{A, A_{0} \in \mathbb{S}}{\mathbb{E}} d_{\mathbb{S}}\left(A, A_{0}\right)=\hat{C} \frac{\operatorname{vol}(\mathcal{S})}{\operatorname{vol}(\mathbb{S})^{2}}
$$


Note that the change of variables $A_{0} \mapsto-A_{0}$ does not change the expected value in this last formula. Moreover, $d_{\mathbb{S}}\left(A, A_{0}\right)+d_{\mathbb{S}}\left(A,-A_{0}\right)=\pi$ for all $A, A_{0} \in \mathbb{S}$. Thus,

$$
\begin{aligned}
\frac{\operatorname{vol}(\mathcal{S})}{\operatorname{vol}(\mathbb{S})^{2}} 2 \hat{C} & =\underset{A, A_{0} \in \mathbb{S}}{\mathbb{E}} d_{\mathbb{S}}\left(A, A_{0}\right)+\underset{A, A_{0} \in \mathbb{S}}{\mathbb{E}} d_{\mathbb{S}}\left(A,-A_{0}\right) \\
& =\underset{A, A_{0} \in \mathbb{S}}{\mathbb{E}}\left(d_{\mathbb{S}}\left(A, A_{0}\right)+d_{\mathbb{S}}\left(A,-A_{0}\right)\right)=\pi,
\end{aligned}
$$

proving that

$$
\hat{C}=\frac{\operatorname{vol}(\mathbb{S})^{2} \pi}{2 \operatorname{vol}(\mathcal{S})} .
$$

From (53) we conclude that for any measurable nonnegative function $\phi$ we have

$$
I_{\phi}=\frac{\pi}{2} \underset{(A, \dot{A}) \sim \mathscr{U}(\mathcal{S})}{\mathbb{E}} \phi(A, \dot{A}),
$$

as wanted.

Proof of Theorem 2.29. Consider the measurable function $\phi: \mathcal{S} \rightarrow[0, \infty]$ defined by

$$
\phi(A, \dot{A})=\frac{1}{n} \sum_{(\lambda, v): A v=\lambda v} \mu(A, \lambda, v)\|(\dot{A}, \dot{\lambda}, \dot{v})\|,
$$

for $A \in \mathbb{S}$ and $\dot{A} \in T_{A} \mathbb{S}$ such that $A \notin \Sigma$, where $\dot{\lambda}, \dot{v}$ are the functions of $(A, \dot{A})$ and $(\lambda, v)$ given in Lemma 3.2. (If $A \in \Sigma$ we set $\phi(A, \dot{A})=\infty$.)

From Theorem 2.19, denoting $I=I_{\phi}$, we have (for some constant $C>1$ )

$$
\underset{A, A_{0} \sim \mathcal{N}_{\mathbb{C}^{n \times n}}}{\mathbb{E}} \frac{1}{n} \sum_{\lambda_{0}, v_{0}:} K\left(A, A_{0}, \lambda_{0}, v_{0}\right) \leq C I .
$$

Note that the left-hand side of (55) is the quantity to be bounded in Theorem 2.29. It is therefore enough for us to show that $I \leq 4 n^{2}$. To do so, write $\mathcal{S}_{A}:=\left\{A^{\prime}:\left(A, A^{\prime}\right) \in \mathcal{S}\right\}$ $\subseteq T_{A} \mathbb{S}$ for $A \in \mathbb{S}$. First note that $\mathcal{S}_{\mathcal{A}}$ is just the unit sphere in $T_{A} \mathbb{S}$, so it has a natural volume form inherited from $\mathbb{C}^{n \times n}$ and $\operatorname{vol}\left(\mathcal{S}_{A}\right)$ is independent of $A$. Moreover, the normal Jacobian of the projection $\mathcal{S} \rightarrow \mathbb{S},(A, \dot{A}) \mapsto A$, is constant and equal to $1 / \sqrt{2}$ (this is easy to prove: check that for $\dot{A} \in T_{A} \mathbb{S}$ and the pair of the form $\left(\dot{A}, \dot{A}^{\prime}\right)$ in $T_{\left(A, A^{\prime}\right)} \mathcal{S}$ which is orthogonal to the kernel of the derivative of the projection is $\left(\dot{A},-\mathbb{R} e\left\langle A^{\prime}, \dot{A}\right\rangle A\right)$, then note that the vectors of that form obtained from any orthogonal basis of $T_{A} \mathbb{S}$ whose first element is $A^{\prime}$ are orthogonal and only one of them, $\left(A^{\prime},-A\right)$, changes its norm by $\left.\sqrt{2}\right)$, so we have $\operatorname{vol}(\mathcal{S})=\sqrt{2} \operatorname{vol}(\mathbb{S}) \operatorname{vol}\left(\mathcal{S}_{A}\right)$. From Lemma 9.1 and Theorem 6.1 , we then have

$$
\begin{aligned}
I & =\frac{\pi}{2} \underset{(A, \dot{A}) \sim \mathcal{U}(\mathcal{S})}{\mathbb{E}} \phi(A, \dot{A}) \\
& =\frac{\pi}{\sqrt{2}} \underset{A \in \mathbb{S}}{\mathbb{E}} \frac{1}{n} \sum_{(\lambda, v): A v=\lambda v} \mu(A, \lambda, v) \underset{\dot{A} \in \mathcal{S}_{A}}{\mathbb{E}}\|(\dot{A}, \dot{\lambda}, \dot{v})\| .
\end{aligned}
$$


In order to estimate this last quantity we shall use Lemma 9.2 below. Note first that from Cauchy-Schwarz,

$$
\begin{aligned}
\underset{\dot{A} \in \mathcal{S}_{A}}{\mathbb{E}}\|(\dot{A}, \dot{\lambda}, \dot{v})\| & \leq\left(1+\underset{\dot{A} \in \mathcal{S}_{A}}{\mathbb{E}}\left(|\dot{\lambda}|^{2}+\|\dot{v}\|^{2}\right)\right)^{1 / 2} \\
& \leq\left(1+\frac{1}{n^{2}-1 / 2}\left(1+2 \mu_{F}(A, \lambda, v)^{2}\right)\right)^{1 / 2} \\
& \leq \frac{1}{n \geq 2}\left(9+\frac{16}{n^{2}} \mu_{F}(A, \lambda, v)^{2}\right)^{1 / 2} .
\end{aligned}
$$

It is a simple exercise to check that for positive $x \in \mathbb{R}$ we have $x\left(9+16 x^{2} / n^{2}\right)^{1 / 2} \leq$ $9 n / 8+4 x^{2} / n$. Using this inequality we get, from the inequalities above,

$$
I \leq \frac{\pi}{\sqrt{14}} \underset{A \in \mathbb{S}}{\mathbb{E}} \frac{1}{n} \sum_{(\lambda, v): A v=\lambda v}\left(\frac{9 n}{8}+\frac{4}{n} \mu_{F}(A, \lambda, v)^{2}\right)
$$

We next use Theorem 2.14 (averaging over $A \in \mathbb{S}$ ) and bound this last quantity by

$$
I \leq \frac{\pi}{\sqrt{14}}\left(\frac{9 n}{8}+\frac{4}{n} n^{3}\right)=\frac{\pi}{\sqrt{14}}\left(\frac{9 n}{8}+4 n^{2}\right) \underset{n \geq 2}{\leq} 4 n^{2},
$$

which finishes the proof.

We have used the following technical lemma which is in the spirit of [2]. Note that as pointed out in the proof of Theorem $2.29, \mathcal{S}_{\mathcal{A}}$ is just the unit sphere in the tangent space $T_{A} \mathbb{S}$ and thus has a natural measure inherited from the inner product in $\mathbb{C}^{n \times n}$.

Lemma 9.2. Let $(A, \lambda, v) \in \mathcal{V}$ with $\|A\|_{F}=1$. Define $\mathcal{S}_{A}:=\left\{A^{\prime}:\left(A, A^{\prime}\right) \in \mathcal{S}\right\} \subseteq T_{A} \mathbb{S}$ as in the proof of Theorem 2.29 (that is, $\mathcal{S}_{A}$ is the unit sphere of $T_{A} \mathbb{S}$ ) and, for $\dot{A} \in \mathcal{S}_{A}$, let $\dot{\lambda}, \dot{v}$ be as in Lemma 3.2. Then

$$
\underset{\dot{A} \in \mathcal{S}_{A}}{\mathbb{E}}|\dot{\lambda}|^{2}=\frac{1}{n^{2}-1 / 2}\left(\mu_{\lambda}(A, \lambda, v)^{2}-|\lambda|^{2} / 2\right), \quad \underset{\dot{A} \in \mathcal{S}_{A}}{\mathbb{E}}\|\dot{v}\|^{2}=\frac{1}{n^{2}-1 / 2}\left\|A_{\lambda, v}^{-1}\right\|_{F}^{2} .
$$

In particular, from Proposition 3.3,

$$
\underset{\dot{A} \in \mathcal{S}_{A}}{\mathbb{E}}\left(|\dot{\lambda}|^{2}+\|\dot{v}\|^{2}\right) \leq \frac{1}{n^{2}-1 / 2}\left(1+2\left\|A_{\lambda, v}^{-1}\right\|_{F}^{2}\right)=\frac{1}{n^{2}-1 / 2}\left(1+2 \mu_{F}(A, \lambda, v)^{2}\right) .
$$

Proof. Note that $T_{A} \mathbb{S}$ coincides with the (real) orthogonal complement, with respect to $\Re\langle\cdot, \cdot\rangle_{F}$, of $A \in \mathbb{C}^{n \times n}$. Thus $\operatorname{dim}_{\mathbb{R}}\left(T_{A} \mathbb{S}\right)=2 n^{2}-1$. On this space we consider the push-forward measure of the standard Gaussian distribution on $\mathbb{C}^{n \times n}$ by the orthogonal projection $\mathbb{C}^{n \times n} \rightarrow T_{A} \mathbb{S}$.

Since $T_{A} \mathbb{S}$ has the (real) orthogonal decomposition $\mathbb{R} \sqrt{-1} A \oplus A^{\perp}$, where $\mathbb{R} \sqrt{-1} A$ is the linear real subspace generated by $\sqrt{-1} A$, in particular we conclude that the Gaussian distribution on $T_{A} \mathbb{S}$ coincides with the distribution $t \sqrt{-1} A+\dot{B} \in T_{A} \mathbb{S}$ where $t \sim \mathcal{N}(0,1 / 2)$ and $\dot{B} \sim \mathcal{N}_{A^{\perp}}$ are independent. 
Claim I. Given a linear operator $L: T_{A} \mathbb{S} \rightarrow \mathbb{C}^{k}$, we have

$$
\underset{\dot{A} \in T_{A} \mathbb{S}}{\mathbb{E}}\|L(\dot{A})\|^{2}=\left(n^{2}-1 / 2\right) \underset{\dot{A} \in \mathcal{S}_{A}}{\mathbb{E}}\|L(\dot{A})\|^{2}
$$

The claim follows by integrating in polar coordinates. More precisely,

$$
\begin{aligned}
\underset{\dot{A} \in T_{A} \mathbb{S}}{\mathbb{E}}\|L(\dot{A})\|^{2} & =\frac{1}{\pi^{n^{2}-1 / 2}} \int_{\dot{A} \in T_{A} \mathbb{S}}\|L(\dot{A})\|^{2} e^{-\|\dot{A}\|^{2}} d \dot{A} \\
& =\frac{1}{\pi^{n^{2}-1 / 2}} \int_{0}^{\infty} \rho^{2 n^{2}} e^{-\rho^{2}} d \rho \cdot \int_{\dot{A} \in \mathcal{S}_{A}}\|L(\dot{A})\|^{2} d \dot{A} \\
& =\left(n^{2}-1 / 2\right) \underset{\dot{A} \in \mathcal{S}_{A}}{\mathbb{E}}\|L(\dot{A})\|^{2},
\end{aligned}
$$

where we have used the equalities $\int_{0}^{\infty} \rho^{2 n^{2}} e^{-\rho^{2}} d \rho=\frac{1}{2} \Gamma\left(n^{2}+1 / 2\right)$ and $\operatorname{vol}\left(\mathcal{S}_{A}\right)=$ $2 \pi^{n^{2}-1 / 2} / \Gamma\left(n^{2}-1 / 2\right)$.

Claim II. For $\|v\|=1$, the push-forward measure of the Gaussian distribution on $A^{\perp}$ by the map $f: A^{\perp} \rightarrow v^{\perp}, \dot{A} \mapsto P_{v^{\perp}}(\dot{A} v)$, is the standard Gaussian on $v^{\perp}$.

Note that for all $B \in \mathbb{C}^{n \times n}$, we have $\left\langle u v^{*}, B\right\rangle_{F}=\operatorname{tr}\left(B^{*} u v^{*}\right)=v^{*} B^{*} u=\langle u, B v\rangle$. Then the set $F:=\left\{\dot{w} v^{*}: \dot{w} \in v^{\perp}\right\}$ is a linear subspace of $A^{\perp}$, and the kernel of $f$ is the Hermitian complement of $F$ as a subset of $A^{\perp}$. Since $\left.f\right|_{F}: F \rightarrow v^{\perp}$ is a linear isometry, the claim follows from the characterization of the standard Gaussian distribution.

Claim III. Let $m \in \mathbb{N}$. If $\eta \sim \mathcal{N}_{\mathbb{C}^{m}}$ and $x \in \mathbb{C}^{m}$ then

$$
\underset{\eta \sim \mathcal{N}_{\mathbb{C}^{m}}}{\mathbb{E}}|\langle\eta, x\rangle|^{2}=\|x\|^{2}
$$

The proof of this claim is a standard exercise and is left to the reader.

Now we are ready to prove the lemma. We choose a representative of $v$ such that $\|v\|=1$ and a representative of the left eigenvector $u$ such that $\langle u, v\rangle=1$. Note that this implies by Proposition 3.3 and Lemma 3.2 that

$$
\mu_{\lambda}(A, \lambda, v)=\frac{\|u\|\|v\|}{|\langle u, v\rangle|}=\|u\|, \quad \dot{\lambda}=\langle\dot{A} v, u\rangle, \quad \dot{v}=-A_{\lambda, v}^{-1} P_{v^{\perp}} \dot{A} v .
$$

For the first statement, we have

$$
\begin{aligned}
\underset{\dot{A} \in T_{A} \mathbb{S}}{\mathbb{E}}|\dot{\lambda}|^{2} & =\underset{\dot{A} \in T_{A} \mathbb{S}}{\mathbb{E}}|\langle\dot{A} v, u\rangle|^{2}=\underset{\dot{A} \in T_{A} \mathbb{S}}{\mathbb{E}}\left|\left\langle\dot{A}, u v^{*}\right\rangle_{F}\right|^{2} \\
& =\underset{\dot{B} \in \mathcal{N}_{A^{\perp}}}{\mathbb{E}} \underset{t \sim \mathcal{N}(0,1 / 2)}{\mathbb{E}}\left|\left\langle t \sqrt{-1} A+\dot{B}, u v^{*}\right\rangle_{F}\right|^{2}
\end{aligned}
$$


Since the mixed term of the expansion of $\left|\left\langle t \sqrt{-1} A+\dot{B}, u v^{*}\right\rangle_{F}\right|^{2}$ is linear in $t$, its expected value is zero. Hence,

$$
\begin{aligned}
\underset{\dot{A} \in T_{A} \mathbb{S}}{\mathbb{E}}|\langle\dot{A} v, u\rangle|^{2} & =\underset{\dot{B} \in \mathcal{N}_{A^{\perp}}}{\mathbb{E}} \underset{t \sim \mathcal{N}(0,1 / 2)}{\mathbb{E}}\left(t^{2}\left|\left\langle A, u v^{*}\right\rangle_{F}\right|^{2}+\left|\left\langle\dot{B}, u v^{*}\right\rangle_{F}\right|^{2}\right) \\
& =|\lambda|^{2} / 2+\underset{\dot{B} \in \mathcal{N}_{A^{\perp}}}{\mathbb{E}}\left|\left\langle\dot{B}, \pi_{A^{\perp}}\left(u v^{*}\right)\right\rangle_{F}\right|^{2} .
\end{aligned}
$$

where we have denoted by $\pi_{A^{\perp}}\left(u v^{*}\right)$ the orthogonal projection of $u v^{*}$ onto $A^{\perp}$. With the identification of $A^{\perp}$ and $\mathbb{C}^{n^{2}-1}$ as Hermitian spaces, from Claim III (with $m=n^{2}-1$ ) we conclude that

$$
\underset{\dot{B} \in \mathcal{N}_{A^{\perp}}}{\mathbb{E}}\left|\left\langle\dot{B}, \pi_{A^{\perp}}\left(u v^{*}\right)\right\rangle_{F}\right|^{2}=\left\|\pi_{A^{\perp}}\left(u v^{*}\right)\right\|_{F}^{2}=\left\|u v^{*}\right\|_{F}^{2}-|\lambda|^{2}=\|u\|^{2}-|\lambda|^{2} .
$$

We have thus proved

$$
\underset{\dot{A} \in T_{A} \mathbb{S}}{\mathbb{E}}|\langle\dot{A} v, u\rangle|^{2}=\mu_{\lambda}(A, \lambda, v)^{2}-|\lambda|^{2} / 2
$$

and from Claim I we conclude that

$$
\underset{\dot{A} \in \mathcal{S}_{A}}{\mathbb{E}}|\langle\dot{A} v, u\rangle|^{2}=\frac{1}{n^{2}-1 / 2}\left(\mu_{\lambda}(A, \lambda, v)^{2}-|\lambda|^{2} / 2\right),
$$

as claimed in the lemma. The second statement in the lemma is proved in a very similar fashion. This time we have

$$
\begin{aligned}
\underset{\dot{A} \in T_{A} \mathbb{S}}{\mathbb{E}}|\dot{v}|^{2} & =\underset{\dot{A} \in T_{A} \mathbb{S}}{\mathbb{E}}\left\|A_{\lambda, v}^{-1} P_{v^{\perp}} \dot{A} v\right\|^{2} \\
& =\underset{\dot{B} \in \mathcal{N}_{A^{\perp}}}{\mathbb{E}} \underset{t \sim \mathcal{N}(0,1 / 2)}{\mathbb{E}}\left\|A_{\lambda, v}^{-1} P_{v^{\perp}}(t \sqrt{-1} A+\dot{B}) v\right\|^{2} \\
& =\underset{\dot{B} \in \mathcal{N}_{A^{\perp}}}{\mathbb{E}}\left\|A_{\lambda, v}^{-1} P_{v^{\perp}} \dot{B} v\right\|^{2}=\underset{\dot{w} \in \mathcal{N}_{v^{\perp}}}{\mathbb{E}}\left\|A_{\lambda, v}^{-1} \dot{w}\right\|^{2}=\left\|A_{\lambda, v}^{-1}\right\|_{F}^{2},
\end{aligned}
$$

the next to last equality coming from Claim II, and the last from the fact that for any matrix $B \in \mathbb{C}^{n \times n}$ we have $\mathbb{E}_{x \sim \mathcal{N}_{\mathbb{C}} n}\|B x\|^{2}=\|B\|_{F}^{2}$ (note the use of Frobenius instead of operator norm in the last equality: that is a crucial point). The last statement of the lemma then follows from Claim I.

\section{Proof of Theorem 2.33}

\subsection{Proof of (1) and (2) in Theorem 2.33}

Note that (2) is trivial. We thus prove (1). The procedure we suggest to choose $\omega \in \Omega_{n}$ at random is the following (note that each step requires $\mathcal{O}\left(n^{3}\right)$ arithmetic operations or random choices): 
(1) Choose $B \sim \mathcal{N}_{\mathbb{C}^{(n-1) \times(n-1)}}$ and let $U$ be the $Q$ factor in the QR decomposition of $B$. Then multiply $Q$ by the diagonal matrix with entries $r_{i i} /\left|r_{i i}\right|$ where the $r_{i i}$ are the diagonal elements of the $R$ factor. This produces a unitary matrix $U$ uniformly distributed in $\mathcal{U}_{n-1}$ (see for example [39]).

(2) Choose $\lambda \sim \mathcal{N}_{\mathbb{C}}$ and $M \sim \mathcal{N}_{\mathbb{C}^{(n-1) \times n}}$. Let $H \in \mathcal{U}_{n}$ with the last column in $\operatorname{ker}(M)$ (it is trivial to produce such an $H$ by computing the QR decomposition of the matrix whose columns are $\operatorname{ker}(M)$ and the columns of $M$ ). Compute $Q$ as the product of the first $n \times(n-1)$ submatrix of $H$ times $U$. This produces an element with the uniform distribution in the set of $Q \in \mathcal{S}_{n-1}\left(\mathbb{C}^{n}\right)$ such that $(M, Q) \in \mathcal{A}_{n}$.

(3) If $2 \Re(\bar{\lambda} \operatorname{tr}(M Q))>1-|\lambda|^{2}(n-1)$ then discard $\lambda, M, Q$ and repeat (1) and (2).

(4) Choose $w \sim \mathcal{N}_{\mathbb{C}^{n-1}}$.

The only subtle point is that steps (1) and (2) might have to be repeated an arbitrary number of times. The expected number of times that steps (1) and (2) will be repeated is related to $C_{n}$ defined in (23) by

$$
\begin{aligned}
\sum_{k=1}^{\infty} \operatorname{Prob}(\text { step } k \text { is reached }) & =\sum_{k=1}^{\infty} \operatorname{Prob}\left(2 \Re(\bar{\lambda} \operatorname{tr}(M Q))>1-|\lambda|^{2}(n-1)\right)^{k-1} \\
& =\sum_{k=1}^{\infty}\left(1-C_{n}^{-1}\right)^{k-1}=\frac{1}{\left(1-\left(1-C_{n}^{-1}\right)\right)}=C_{n} .
\end{aligned}
$$

10.2. Proof of (3) in Theorem 2.33

We are now prepared for proving (24). Let $\mathbb{1}$ be the characteristic function of the set

$$
\left\{(\lambda, B): 2 \Re(\bar{\lambda} \operatorname{tr}(B)) \leq 1-|\lambda|^{2}(n-1)\right\} \subseteq \mathbb{C} \times \mathbb{C}^{(n-1) \times(n-1)} .
$$

From the definition and Fubini's theorem, for any measurable nonnegative function $\phi$ defined on $\mathcal{V}$, the expected value $\mathbb{E}_{\omega \sim \Omega_{n}} \phi\left(\psi_{n}(\omega)\right)$ equals

$$
\begin{aligned}
& C_{n} \underset{M}{\mathbb{E}} \underset{Q:(M, Q) \in \mathcal{A}_{n}}{\mathbb{E}} \underset{\lambda, w}{\mathbb{E}} \phi\left(\left(\begin{array}{cc}
\lambda & w^{*} \\
0 & M Q+\lambda I_{n-1}
\end{array}\right), \lambda, e_{1}\right) \mathbb{1}(\lambda, M Q) \\
& =C_{n} \underset{M}{\underset{Q:(M, Q) \in \mathcal{A}_{n}}{\mathbb{E}}} \underset{\mathrm{E}}{\mathbb{E}} \alpha(M Q),
\end{aligned}
$$

where $\lambda \sim \mathcal{N}_{\mathbb{C}}, M \sim \mathcal{N}_{\mathbb{C}^{(n-1) \times n}}, w \sim \mathcal{N}_{\mathbb{C}^{n-1}}$ and $\alpha: \mathbb{C}^{(n-1) \times(n-1)} \rightarrow[0, \infty]$ is defined by

$$
\alpha(B)=\underset{\lambda, w}{\mathbb{E}} \phi\left(\left(\begin{array}{cc}
\lambda & w^{*} \\
0 & B+\lambda I_{n-1}
\end{array}\right), \lambda, e_{1}\right) \mathbb{1}(\lambda, B) .
$$

We are then under the hypotheses of Corollary 6.7. Using this result we obtain

$$
\underset{w \sim \Omega_{n}}{\mathbb{E}} \phi\left(\psi_{n}(w)\right)=\frac{C_{n}}{\Gamma(n)} \underset{B \sim \mathcal{N}_{\mathbb{C}^{(n-1) \times(n-1)}}}{\mathbb{E}} \alpha(B)|\operatorname{det}(B)|^{2} .
$$


With the change of variables $B+\lambda I_{n-1}=D$, which implies

$$
\|B\|_{F}^{2}=\|D\|_{F}^{2}+(n-1)|\lambda|^{2}-2 \Re(\bar{\lambda} \operatorname{tr}(D)),
$$

this last expression equals

$\frac{C_{n}}{\Gamma(n)} \underset{\lambda, w, D}{\mathbb{E}} \phi\left(\left(\begin{array}{cc}\lambda & w^{*} \\ 0 & D\end{array}\right), \lambda, e_{1}\right)\left|\operatorname{det}\left(D-\lambda I_{n-1}\right)\right|^{2} e^{-|\lambda|^{2}(n-1)+2 \Re(\bar{\lambda} \operatorname{tr}(D))} \mathbb{1}\left(\lambda, D-\lambda I_{n-1}\right)$,

where $D \sim \mathcal{N}_{\mathbb{C}^{(n-1) \times(n-1)}}$. Now, note that

$$
\mathbb{1}\left(\lambda, D-\lambda I_{n-1}\right) \neq 0 \Leftrightarrow e^{-|\lambda|^{2}(n-1)+2 \Re(\bar{\lambda} \operatorname{tr}(D))} \leq e .
$$

We have thus proved

$$
\begin{aligned}
\underset{w \sim \Omega_{n}}{\mathbb{E}}\left(\phi\left(\psi_{n}(w)\right)\right. & \leq \frac{e C_{n}}{\Gamma(n)} \underset{\lambda, w, D}{\mathbb{E}} \phi\left(\left(\begin{array}{cc}
\lambda & w^{*} \\
0 & D
\end{array}\right), \lambda, e_{1}\right)\left|\operatorname{det}\left(D-\lambda I_{n-1}\right)\right|^{2} \\
& =e n C_{n} \underset{A \sim \mathcal{N}_{\mathbb{C}^{n \times n}}}{\mathbb{E}} \frac{1}{n} \sum_{\lambda, v: A v=\lambda v} \phi(A, \lambda, v) .
\end{aligned}
$$

This proves claim (3) in Theorem 2.33.

We prove the following (nonsharp) bound for the value of $C_{n}$.

Lemma 10.1. With the notations above,

$$
C_{n} \leq 4 n \text {. }
$$

Proof. Note that if $0<|\lambda| \leq(n-1)^{-1 / 2}$, then for any nonzero $M \in \mathbb{C}^{(n-1) \times n}$ we have

$$
\left.\left.\underset{Q}{\operatorname{Prob}}\left(2 \Re(\bar{\lambda} \operatorname{tr}(M Q)) \leq 1-|\lambda|^{2}(n-1)\right) \geq \underset{Q}{\operatorname{Prob}(2 \Re(\bar{\lambda}} \operatorname{tr}(M Q)\right) \leq 0\right)=\frac{1}{2},
$$

the last equality coming from the linearity of the trace. We thus have

$\underset{\lambda, M, Q}{\operatorname{Prob}}\left(2 \Re(\bar{\lambda} \operatorname{tr}(M Q)) \leq 1-|\lambda|^{2}(n-1)\right) \geq \frac{1}{\pi} \int_{|\lambda|<(n-1)^{-1 / 2}} \frac{e^{-|\lambda|^{2}}}{2} d \lambda=\frac{1-e^{-1 /(n-1)}}{2}$.

We thus have

$$
C_{n} \leq \frac{2}{1-e^{-1 /(n-1)}} \leq 4(n-1) \leq 4 n
$$

as claimed. 


\section{Proof of Theorem 1.1}

Consider the following algorithm.

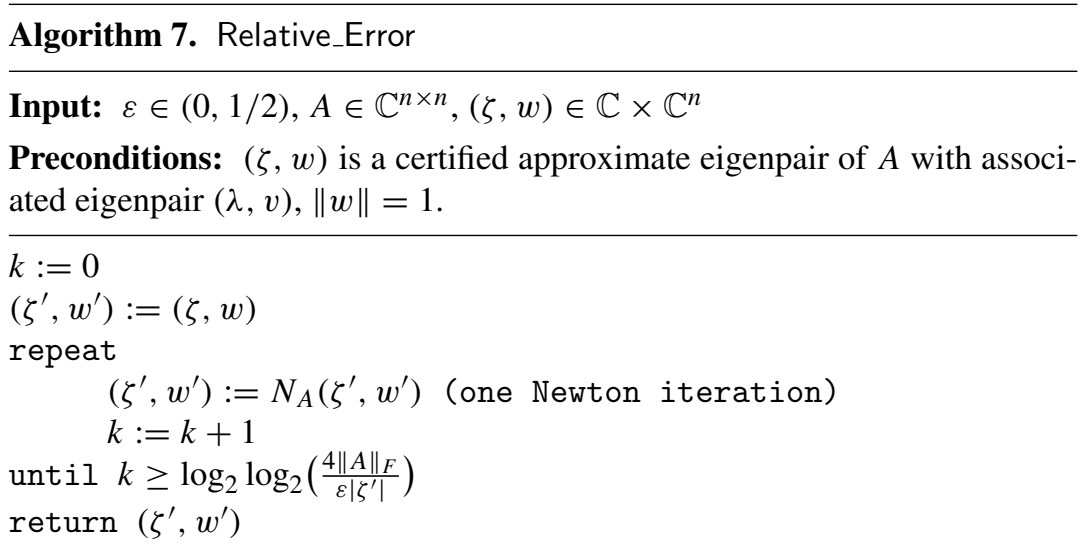

Output: $\left(\zeta^{\prime}, w^{\prime}\right) \in \mathbb{C} \times \mathbb{C}^{n}$

Postconditions: The algorithm halts if $\lambda \neq 0$. In this case, $\left(\zeta^{\prime}, w^{\prime}\right)$ is an approximate eigenpair of $A$ with associated eigenpair $(\lambda, v)$, and $d_{\mathbb{S}}\left(w^{\prime}, v\right) \leq \varepsilon$, and moreover $\left|\zeta^{\prime}-\lambda\right| \leq \varepsilon|\lambda|$ and $\operatorname{dist}_{A}((\zeta, w),(\lambda, v)) \leq$ $(1 / 2)^{2^{2^{k}-1}} c^{*} / \mu(A, \lambda, v)$.

By hypothesis,

$$
\operatorname{dist}_{A}((\zeta, w),(\lambda, v)) \leq \frac{c_{*}}{\mu(A, \lambda, v)}<1 .
$$

Hence, $|\zeta-\lambda| /\|A\|_{F} \leq \operatorname{dist}_{A}((\zeta, w),(\lambda, v))<1$, and the same bound holds with $\zeta$ replaced by $\zeta^{\prime}$ at all the iterations of the algorithm (by Definition 2.11). Using now $|\lambda| \leq\|A\|_{F}$, we deduce that $\left|\zeta^{\prime}\right| \leq 2\|A\|_{F}$. Hence, at the end of the repeat loop the value $k$ satisfies

$$
2^{2^{k}} \geq \frac{4\|A\|_{F}}{\varepsilon\left|\zeta^{\prime}\right|} \geq \frac{2}{\varepsilon}
$$

This inequality implies that after $k$ iterations of the loop we have, from the definition of approximate eigenpair and the bound (58),

$$
\frac{\left|\zeta^{\prime}-\lambda\right|}{\|A\|_{F}} \leq \operatorname{dist}_{A}\left(\left(\zeta^{\prime}, w^{\prime}\right),(\lambda, v)\right) \leq \frac{c_{*}}{\mu(A, \lambda, v)}\left(\frac{1}{2}\right)^{2^{k}-1} \leq\left(\frac{1}{2}\right)^{2^{k}-1}=\frac{2}{2^{2^{k}}} \leq \varepsilon .
$$

In particular, $d_{\mathbb{S}}\left(w^{\prime}, v\right) \leq \varepsilon$ as we wanted. On the other hand, the first inequality in (59) implies

$$
2^{2^{k}-1}\left|\zeta^{\prime}\right|-\|A\|_{F} \geq 2\|A\|_{F} / \varepsilon-\|A\|_{F} \geq\|A\|_{F} / \varepsilon
$$


the last since $\varepsilon<1$. We now use this inequality together with (60) to obtain

$$
\frac{\left|\zeta^{\prime}-\lambda\right|}{|\lambda|} \leq \frac{\|A\|_{F}}{|\lambda| 2^{2^{k}-1}} \leq \frac{\|A\|_{F}}{\left(\left|\zeta^{\prime}\right|-\frac{\|A\|_{F}}{2^{2^{k}-1}}\right) 2^{2^{k}-1}}=\frac{\|A\|_{F}}{2^{2^{k}-1}\left|\zeta^{\prime}\right|-\|A\|_{F}} \leq \varepsilon,
$$

i.e., $\left|\zeta^{\prime}-\lambda\right| \leq \varepsilon|\lambda|$.

It remains to show that Relative_Error halts provided $\lambda \neq 0$ and to estimate its average running time when $A$ is drawn from $\mathcal{N}_{\mathbb{C}^{n \times n}}$. For this, we note that as soon as

$$
k \geq \log _{2} \log _{2}\left(\frac{8\|A\|_{F}}{\varepsilon|\lambda|}\right)
$$

we shall have (using (60))

$$
\left|\zeta^{\prime}\right| \geq|\lambda|-\|A\|_{F} / 2^{2^{k}-1} \geq|\lambda|-\varepsilon|\lambda| / 4=|\lambda|(1-\varepsilon / 4) .
$$

Therefore, we will also have

$$
\log _{2} \log _{2}\left(\frac{4\|A\|_{F}}{\varepsilon\left|\zeta^{\prime}\right|}\right) \leq \log _{2} \log _{2}\left(\frac{4\|A\|_{F}}{\varepsilon|\lambda|(1-\varepsilon / 4)}\right) \leq \log _{2} \log _{2}\left(\frac{8\|A\|_{F}}{\varepsilon|\lambda|}\right) \leq k
$$

Hence, the stopping condition will hold after at most

$$
\log _{2} \log _{2}\left(\frac{8\|A\|_{F}}{\varepsilon|\lambda|}\right) \leq \log _{2} \log _{2}\left(\frac{8\|A\|_{F}\left\|A^{-1}\right\|}{\varepsilon}\right)
$$

iterations (we have used $|\lambda|^{-1} \leq\left\|A^{-1}\right\|$ for $\lambda$ is an eigenvalue of $A$ ).

We finally estimate the average cost of Relative_Error. Since each iteration of the repeat loop requires $\mathcal{O}\left(n^{3}\right)$ operations, this cost is at most $\mathcal{O}\left(n^{3}\right)$ times

$$
\underset{A \sim \mathcal{N}_{\mathbb{C}^{n \times n}}}{\mathbb{E}} \log _{2} \log _{2}\left(\frac{8\|A\|_{F}\left\|A^{-1}\right\|}{\varepsilon}\right) \leq \log _{2} \log _{2}\left(\frac{8}{\varepsilon} \underset{\mathcal{N}_{\mathbb{C}^{n \times n}}}{\mathbb{E}}\|A\|_{F}\left\|A^{-1}\right\|\right),
$$

where we have used Jensen's inequality. Bounds for the expected value of $\left\|A^{-1}\right\|$ when $A \sim \mathcal{N}_{\mathbb{C}^{n \times n}}$ are known (see for example [19, Prop. 4.22]), which, together with the Cauchy-Schwarz inequality $\mathbb{E}(f g) \leq\left(\mathbb{E}\left(f^{2}\right) \mathbb{E}\left(g^{2}\right)\right)^{1 / 2}$, implies

$$
\underset{A \sim \mathcal{N}_{\mathbb{N}^{n \times n}}^{\mathbb{E}}}{\mathbb{E}}\|A\|_{F}\left\|A^{-1}\right\| \leq \sqrt{n^{2} \frac{e(n+1)}{2}} \leq 2 n^{3 / 2}
$$

The statement follows.

Acknowledgments. We want to thank Jim Demmel for helpful comments and Froilán Dopico for suggesting that we work out the case of relative error. P. Bürgisser, F. Cucker and M. Shub want to thank the Simons Institute where they spent some time while this work was being carried out.

D. Armentano was partially supported by Agencia Nacional de Investigación e Innovación (ANII), Uruguay, and by CSIC group 618.

C. Beltrán was partially suported by the research projects MTM2010- 16051, MTM2014-57590 and MTM2015-68805-REDT from Spanish Ministry of Economy and by grant 21.SI01.64658 from Banco Santander and Universidad de Cantabria.

P. Bürgisser was partially funded by DFG research grant BU 1371/2-2.

F. Cucker was partially funded by a GRF grant from the Research Grants Council of the Hong Kong SAR (project number CityU 11300714). 


\section{References}

[1] Amelunxen, D., Bürgisser, P.: Probabilistic analysis of the Grassmann condition number. Found. Comput. Math. 15, 3-51 (2015) Zbl 1309.90075 MR 3303690

[2] Armentano, D.: Stochastic perturbations and smooth condition numbers. J. Complexity 26, 167-171 (2010) Zbl 1269.15003 MR 2607730

[3] Armentano, D.: Complexity of path-following methods for the eigenvalue problem. Found. Comput. Math. 14, 185-236 (2014) Zbl 1302.65085 MR 3179583

[4] Armentano, D.: Beltrán, C,, Bürgisser, P., Cucker, F., Shub, M.: Condition length and complexity for the solution of polynomial systems. Found. Comput. Math. 16, 1401-1422 (2016) Zbl 1358.65031 MR 3579713

[5] Armentano, D., Cucker, F.: A randomized homotopy for the Hermitian eigenpair problem. Found. Comput. Math. 15, 281-312 (2015) Zbl 1317.65096 MR 3303697

[6] Bai, Z., Demmel, J. W., Gu, M.: An inverse free parallel spectral divide and conquer algorithm for nonsymmetric eigenproblems. Numer. Math. 76, 279-308 (1997) Zbl 0876.65021 MR 1452510

[7] Batterson, S.: Convergence of the Francis shifted $Q R$ algorithm on normal matrices. Linear Algebra Appl. 207, 181-195 (1994) Zbl 0808.65030 MR 1283957

[8] Batterson, S., Smillie, J.: Rayleigh quotient iteration fails for nonsymmetric matrices. Appl. Math. Lett. 2, 19-20 (1989) Zbl 0706.65037 MR 0989851

[9] Batterson, S., Smillie, J.: Rayleigh quotient iteration for nonsymmetric matrices. Math. Comp. 55, 169-178 (1990) Zbl 0701.65027 MR 1023041

[10] Beckermann, B., Golub, G. H., Labahn, G.: On the numerical condition of a generalized Hankel eigenvalue problem. Numer. Math. 106, 41-68 (2007) Z Zbl 1121.65036 MR 2286006

[11] Beltrán, C., Leykin, A.: Robust certified numerical homotopy tracking. Found. Comput. Math. 13, 253-295 (2013) Zbl 1267.14075 MR 3032682

[12] Beltrán, C., Pardo, L. M.: Smale's 17th problem: average polynomial time to compute affine and projective solutions. J. Amer. Math. Soc. 22, 363-385 (2009) Zbl 1206.90173 MR 2476778

[13] Beltrán, C., Pardo, L. M.: Fast linear homotopy to find approximate zeros of polynomial systems. Found. Comput. Math. 11, 95-129 (2011) Zbl 1232.65075 MR 2754191

[14] Blum, L., Cucker, F., Shub, M., Smale, S.: Complexity and Real Computation. Springer, New York (1998) Zbl 0948.68068 MR 1479636

[15] Breiding, P.: An adaptive linear homotopy method to approximate eigenpairs of homogeneous polynomial systems. arXiv:1512.03284 (2015)

[16] Briquel, I., Cucker, F., Peña, J., Roshchina, V.: Fast computation of zeros of polynomial systems with bounded degree under finite-precision. Math. Comp. 83, 1279-1317 (2014) Zbl 1291.65158 MR 3167459

[17] Bürgisser, P.: Smoothed analysis of condition numbers. In: Proc. International Congress of Mathematicians (Hyderabad, 2010), Vol. IV, Hindustan Book Agency, New Delhi, 2609-2633 (2010) Zbl 1231.65095 MR 2827986

[18] Bürgisser, P., Cucker, F.: On a problem posed by Steve Smale. Ann. of Math. 174, 1785-1836 (2011) Zbl 1248.65047 MR 2846491

[19] Bürgisser, P., Cucker, F.: Condition. Grundlehren Math. Wiss. 349, Springer, Berlin (2013) Zbl 1280.65041 MR 3098452

[20] Choi, K. P.: On the medians of gamma distributions and an equation of Ramanujan. Proc. Amer. Math. Soc. 121, 245-251 (1994) Zbl 0803.62007 MR 1195477

[21] Chu, M. T.: A simple application of the homotopy method to symmetric eigenvalue problems. Linear Algebra Appl. 59, 85-90 (1984) Zbl 0544.65019 MR 0743047 
[22] Dedieu, J.-P., Malajovich, G., Shub, M.: On the curvature of the central path of linear programming theory. Found. Comput. Math. 5, 145-171 (2005) Zbl 1119.90370 MR 2149414

[23] Dedieu, J.-P., Malajovich, G., Shub, M.: Adaptive step size selection for homotopy methods to solve polynomial equations. IMA J. Numer. Anal. 33, 1-29 (2013) Zbl 1266.65080 MR 3020948

[24] Dedieu, J.-P., Shub, M.: Multihomogeneous Newton methods. Math. Comp. 69, 1071-1098 (2000) Zbl 0951.65049 MR 1752092

[25] Demmel, J. W.: Applied Numerical Linear Algebra. SIAM (1997) Zbl 0879.65017 MR 1463942

[26] Demmel, J. W., Dumitriu, I., Holtz, O.: Fast linear algebra is stable. Numer. Math. 108, 59-91 (2007) Zbl 1133.65015 MR 2350185

[27] Evans, L. C., Gariepy, R. F.: Measure Theory and Fine Properties of Functions. CRC Press, Boca Raton, FL (1992) Zbl 0804.28001 MR 1158660

[28] Federer, H.: Geometric Measure Theory. Grundlehren Math. Wiss. 153, Springer, Berlin (1969) Zbl 0176.00801 MR 0257325

[29] Golub, G. H., van Loan, C. F.: Matrix Computations. 3rd ed., Johns Hopkins Univ. Press, Baltimore, MD (1996) Zbl 1118.65316 MR 1417720

[30] Howard, R.: The kinematic formula in Riemannian homogeneous spaces. Mem. Amer. Math. Soc. 106, no. 509, 69 pp. (1993) Zbl 0810.53057 MR 1169230

[31] Kahan, W.: Accurate eigenvalues of a symmetric tridiagonal matrix. Technical report no. CS41, Computer Science Department, Stanford Univ.

[32] Kostlan, E.: Complexity theory of numerical linear algebra. J. Comput. Appl. Math. 22, 219230 (1988) Zbl 0645.65019 MR 0956504

[33] Lahaye, E.: Une méthode de résolution d'une catégorie d'équations transcendantes. C. R. Acad. Sci. Paris 198, 1840-1842 (1934) Zbl 0009.17504

[34] Lairez, P.: A deterministic algorithm to compute approximate roots of polynomial systems in polynomial average time. Found. Comput. Math. 17, 1265-1292 (2017) Zbl 06814854 MR 3709332

[35] Li, T.-Y.: Numerical solution of polynomial systems by homotopy continuation methods. In: $\mathrm{Ph}$. Ciarlet and F. Cucker (eds.), Handbook of Numerical Analysis, Vol. XI, North-Holland, Amsterdam, 209-304 (2003) Zbl 1059.65046 MR 2009773

[36] Li, T.-Y., Sauer, T.: Homotopy method for generalized eigenvalue problems $A x=\lambda B x$. Linear Algebra Appl. 91, 65-74 (1987) Zbl 0621.65028 MR 0888479

[37] Li, T.-Y., Zeng, Z. G.: Homotopy-determinant algorithm for solving nonsymmetric eigenvalue problems. Math. Comp. 59, 483-502 (1992) Zbl 0783.65034 MR 1151113

[38] Li, T.-Y., Zeng, Z. G., Cong, L.: Solving eigenvalue problems of real nonsymmetric matrices with real homotopies. SIAM J. Numer. Anal. 29, 229-248 (1992) Zbl 0749.65028 MR 1149095

[39] Mezzadri, F.: How to generate random matrices from the classical compact groups. Notices Amer. Math. Soc. 54, 592-604 (2007) Zbl 1156.22004 MR 2311982

[40] Nakatsukasa, Y., Higham, N. J.: Stable and efficient spectral divide and conquer algorithms for the symmetric eigenvalue decomposition and the SVD. SIAM J. Sci. Comput. 35, A1325A1349 (2013) Zbl 1326.65049 MR 3054594

[41] Pfrang, C. W., Deift, P., Menon, G.: How long does it take to compute the eigenvalues of a random symmetric matrix? In: Random Matrix Theory, Interacting Particle Systems, and Integrable Systems, Math. Sci. Res. Inst. Publ. 65, Cambridge Univ. Press, New York, 411442 (2014) Zbl 1326.65050 MR 3380694

[42] Segal, I. E., Kunze, R. A.: Integrals and Operators. 2nd ed., Springer, Berlin (1978) Zbl 0373.28001 MR 0486380 
[43] Shub, M.: Some remarks on Bézout's Theorem and complexity theory. In: From Topology to Computation: Proceedings of the Smalefest (Berkeley, CA, 1990), Springer, New York, 443-455 (1993) Zbl 0811.13021 MR 1246139

[44] Shub, M.: Complexity of Bézout's Theorem VI: geodesics in the condition (number) metric. Found. Comput. Math. 9, 171-178 (2009) Zbl 1175.65060 MR 2496558

[45] Shub, M., Smale, S.: Complexity of Bézout's Theorem I: geometric aspects. J. Amer. Math. Soc. 6, 459-501 (1993) Zbl 0821.65035 MR 1175980

[46] Shub, M., Smale, S.: Complexity of Bézout's Theorem II: volumes and probabilities. In: F. Eyssette and A. Galligo (eds.), Computational Algebraic Geometry, Progr. Math. 109, Birkhäuser, 267-285 (1993) Zbl 0851.65031 MR 1230872

[47] Shub, M., Smale, S.: Complexity of Bézout's Theorem III: condition number and packing. J. Complexity 9, 4-14 (1993) Zbl 0846.65018 MR 1213484

[48] Shub, M., Smale, S.: Complexity of Bézout's Theorem IV: probability of success; extensions. SIAM J. Numer. Anal. 33, 128-148 (1996) Zbl 0843.65035 MR 1377247

[49] Shub, M., Smale, S.: Complexity of Bézout's Theorem V: polynomial time. Theoret. Computer Sci. 133, 141-164 (1994) Zbl 0846.65022 MR 1294430

[50] Smale, S.: Newton's method estimates from data at one point. In: R. Ewing et al. (eds.), The Merging of Disciplines: New Directions in Pure, Applied, and Computational Mathematics, Springer, 185-196 (1986) Zbl 0613.65058 MR 0870648

[51] Smale, S.: Complexity theory and numerical analysis. In: A. Iserles (ed.), Acta Numerica, Cambridge Univ. Press, 523-551 (1997) Zbl 0883.65125 MR 1489262

[52] Smale, S.: Mathematical problems for the next century. Math. Intelligencer 20, 7-15 (1998) Zbl 0947.01011 MR 1631413

[53] Spielman, D. A., Teng, S.-H.: Smoothed analysis of algorithms. In: Proc. International Congress of Mathematicians (Beijing, 2002), Vol. I, Higher Ed. Press, Beijing, 597-606 (2002) Zbl 1056.65148 MR 1989210

[54] Spielman, D. A., Teng, S.-H.: Smoothed analysis: Why the simplex algorithm usually takes polynomial time. J. ACM 51, 385-463 (2004) Zbl 1192.90120 MR 2145860

[55] Spielman, D. A., Teng, S.-H.: Smoothed analysis: An attempt to explain the behavior of algorithms in practice. Comm. ACM 52, 77-84 (2009)

[56] van Loan, C. F.: On estimating the condition of eigenvalues and eigenvectors. Linear Algebra Appl. 88-89, 715-732 (1987) Zbl 0631.65030 MR 0882468

[57] von Neumann, J., Goldstine, H. H.: Numerical inverting matrices of high order, II. Proc. Amer. Math. Soc. 2, 188-202 (1951) Zbl 0043.12301 MR 0041539

[58] Weyl, H.: Das asymptotische Verteilungsgesetz der Eigenwerte linearer partieller Differentialgleichungen. Math. Ann. 71, 441-479 (1912) JFM 43.0436.01 MR 1511670 